\title{
Synthesis and Conformational Properties of 3,4-Difluoro-L-prolines
}

\author{
Gert-Jan Hofman, $^{\dagger, \ddagger}$ Emile Ottoy, ${ }^{\ddagger}$ Mark E. Light, ${ }^{\dagger}$ Bruno Kieffer, $^{\S}$ Jose C. Martins, ${ }^{\ddagger}$ Ilya Kuprov, ${ }^{\dagger}$ \\ Davy Sinnaeve, ${ }^{\ddagger}, \perp_{(0)}$ and Bruno Linclau, ${ }^{*}, \dagger$
}

\author{
${ }^{\dagger}$ School of Chemistry, University of Southampton, Highfield, Southampton SO17 1BJ, United Kingdom \\ ${ }^{\ddagger}$ Department of Organic and Macromolecular Chemistry, Ghent University, Campus Sterre, S4, Krijgslaan 281, Ghent B-9000, \\ Belgium \\ ${ }^{\S}$ Biomolecular NMR, University of Strasbourg, IGBMC, CNRS UMR 7104, INSERM U1258, 1 rue Laurent Fries/BP 10142, \\ Illkirch Cedex 67404, France
}

\section{Supporting Information}

ABSTRACT: Fluorinated proline derivatives have found diverse applications in areas ranging from medicinal chemistry over structural biochemistry to organocatalysis. Depending on the stereochemistry of monofluorination at the proline 3- or 4-position, different effects on the conformational properties of proline (ring pucker, cis/trans isomerization) are introduced. With fluorination at both 3- and 4-positions, matching or mismatching effects can occur depending on the relative stereochemistry. Here we report, in full, the syntheses and conformational properties of three out of the four possible 3,4difluoro-L-proline diastereoisomers. The yet unreported conformational properties are described for $(3 S, 4 S)$ - and $(3 R, 4 R)$-difluoro-L-proline, which are shown to bias ring pucker and cis/trans ratios on the same order of magnitude as their respective monofluorinated progenitors, although with significantly faster amide cis/trans isomerization rates. The reported analogues

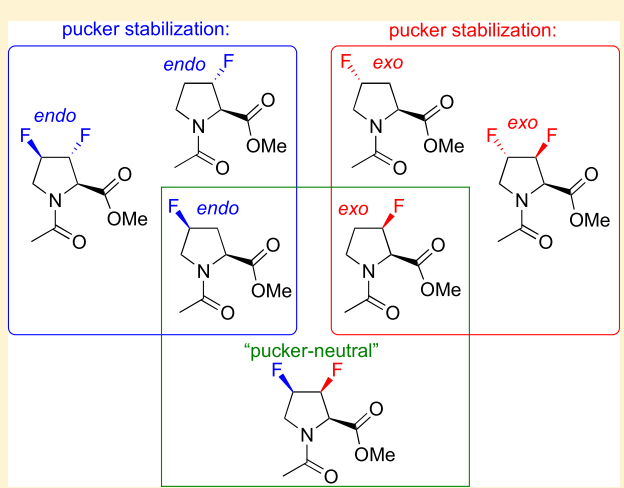
thus expand the scope of available fluorinated proline analogues as tools to tailor proline's distinct conformational and dynamical properties, allowing for the interrogation of its role in, for instance, protein stability or folding.

\section{INTRODUCTION}

Fluorination of organic molecules has proven to be a highly useful tool for the manipulation of their conformational and electronic properties with minimal steric effects. ${ }^{1-7}$ Fluorination of the L-proline ring has been heavily exploited for conformational control of its ring pucker. ${ }^{8}$ For example, the five-membered proline ring conformation can be biased to either a $C^{\gamma}$ exo or a $C^{\gamma}$ endo pucker by introducing a (4R)fluoro group (1, Figure 1) or a (4S)-fluoro group (2), respectively, an effect attributed to $\sigma_{\mathrm{CH}} \rightarrow \sigma^{*}{ }_{\mathrm{CF}}$ hyperconjugation interactions. ${ }^{9}$ Besides a ring pucker, fluorination also strongly influences the cis/trans ratio of the Xaa-Pro peptide bond relative to proline in a solvent-dependent way. ${ }^{10}$ The inductive effect of fluorine reduces the capacity for the nitrogen lone pair to conjugate with the amide carbonyl group and thus to contribute to the double bond character of the amide bond. As a consequence, the rotational energy barrier is decreased and accelerated cis/trans isomerization is observed. ${ }^{11-13}$ The same effect renders fluorinated prolines less basic $^{11,13,14}$ and the carboxylic acid group more acidic. ${ }^{15}$

The combination of both conformational and dynamical effects make fluoroprolines valuable tools for determining the significance of proline's unique structural properties within proteins or peptides. ${ }^{8,14}$ Nevertheless, the first syntheses of (4R)-FPro 1 and (4S)-FPro 2 date back to $1965,{ }^{16}$ although it took until the late $1990 \mathrm{~s}$ for this potential to be fully a) $\quad F,(R)$

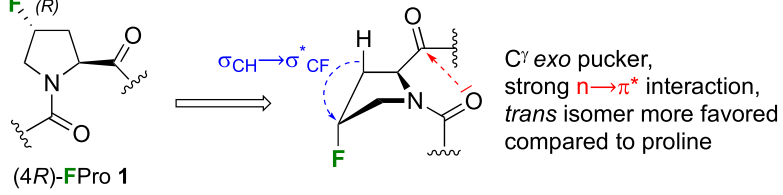

b)
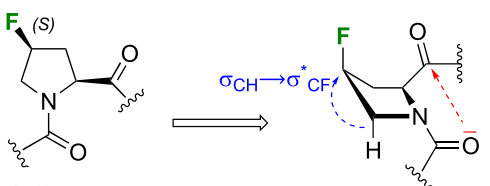

$\mathrm{C}^{\gamma}$ endo pucker weak $n \rightarrow \pi^{*}$ interaction, trans isomer less favored compared to proline (4S)-FPro 2

c)

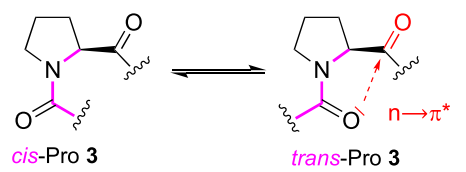

$\mathrm{n} \rightarrow \pi^{*}$ interaction stabilizes the trans isomer

Figure 1. (a) (4R)-FPro 1 adopts an $C^{\gamma}$ exo pucker. (b) (4S)-FPro 2 adopts an $\mathrm{C}^{\gamma}$ endo pucker. (c) The $n \rightarrow \pi^{*}$ interaction stabilizes the trans isomer.

recognized. In a landmark study investigating the mechanism behind collagen stability, ${ }^{9,17,18}$ Raines and co-workers applied fluoroprolines to revise the origins behind the extraordinary

Received: November 15, 2018

Published: February 19, 2019 
thermostability of this protein, which forms triple helices out of Pro-Hyp-Gly repeats. Replacing (4R)-4-hydroxyproline (Hyp) with (4R)-FPro 1 led to a more thermostable collagen mimic, which, since fluorine is a weak hydrogen bond acceptor, disproved that a hydrogen bond network involving the hydroxyl moiety of Hyp induces collagen stability. In contrast, replacing Hyp with (4S)-FPro 2 led to less stable collagen mimics. Since fluorine is more electronegative than a hydroxyl group, (4R)-FPro favors the $C^{\gamma}$ exo pucker more strongly than Hyp, and because (4S)-FPro favors the $\mathrm{C}^{\gamma}$ endo pucker, this revealed that it is the strong preference for the $\mathrm{C}^{\gamma}$ exo pucker of Hyp that plays a key role for collagen stability. This ring pucker preorganizes the dihedral angles in such a way that a favorable $n \rightarrow \pi^{*}$ interaction is promoted between the carbonyl groups of two adjacent peptide bonds, favoring the trans amide bond rotamer. ${ }^{19}$ Interestingly, the ring pucker of the Pro residue preceding Hyp is also relevant for collagen stability, ${ }^{20}$ which has equally been investigated using both 4- and 3monofluorinated proline variants. ${ }^{21}$

The case of collagen initiated many other demonstrations of the potential of proline fluorination to investigate the distinct structural and dynamical properties of proline residues within peptides and proteins, exploiting both the modulations of proline structure and cis/trans isomerization kinetics. ${ }^{8}$ Indeed, modulating these properties by fluorination, rather than just fully eliminating them by mutating proline to nonproline residues, ${ }^{22}$ can provide a more elegant approach toward uncovering the functional significance of proline's unique properties. Moreover, the introduction of fluorine allows the use of ${ }^{19} \mathrm{~F}$ NMR as a powerful means to monitor residuespecific information. The exceptionally high responsivity of the ${ }^{19} \mathrm{~F}$ nucleus to changes in its (local) environment, in addition to the sparsity of the ${ }^{19} \mathrm{~F}$ spectrum, make ${ }^{19} \mathrm{~F}$ NMR a very attractive means to monitor protein structural and dynamical changes, enzyme catalysis, and ligand binding. ${ }^{23-28}$ Despite these clear advantages and earlier suggestions, ${ }^{29-31}$ to the best of our knowledge, there are only a very limited number of reports involving the full potential of ${ }^{19} \mathrm{~F}$ NMR in a fluoroproline peptide context. ${ }^{32,33}$ However, if the FPro residue is to be used purely as a ${ }^{19} \mathrm{~F}$ NMR probe, the conformationally perturbing effects of fluorine must be carefully considered. We recently introduced (3S,4R)-3,4difluoroproline $((3 S, 4 R)$-FPro) 4 (Figure 2$)$ where the two
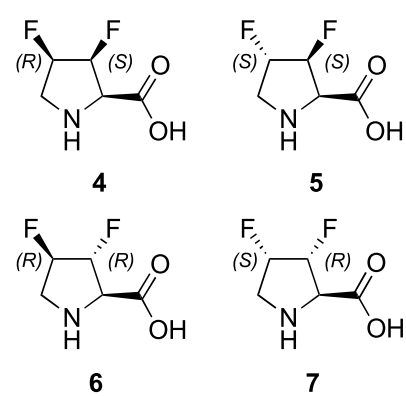

Figure 2. Structures of the targeted 3,4-difluoroprolines.

fluorines have opposing preorganizing effects, thus resulting in a proline analogue with minimal conformational bias and minimal homonuclear coupling complications for ${ }^{19} \mathrm{~F}$ NMR purposes. $^{34}$

Given the well-demonstrated importance of having fluoroprolines available with matching conformational, kinetic, and NMR properties for the application at hand, the synthesis of novel fluorinated variants in an optically pure form continues to be of interest. ${ }^{35}$ In addition, regardless of whether such applications require conformationally neutral, $\mathrm{C}^{\gamma}$ exo or $\mathrm{C}^{\gamma}$ endo pucker promoting fluoroprolines, the availability of more than one variant with similar conformational properties, but well-separated ${ }^{19} \mathrm{~F}$ NMR chemical shifts, is of interest for site-specific multiresidue-labeling strategies of proteins, especially in the case of low-complexity sequences found in prolinerich proteins such as collagen, but also many transcriptional activators. Hence, we envisaged a convenient synthesis of the 3,4-difluoro-L-prolines 4-7 (Figure 2), in order to expand the toolbox of proline analogues.

There exists only a limited precedence for such difluorinated proline analogues (Scheme 1). A Novartis patent describes the synthesis of $\mathrm{N}$-Boc-7 in 14 steps from commercially available 3,4-dehydroproline $8 .^{36}$ After epoxidation and acid-catalyzed epoxide opening, the key fluorination steps involve DASTmediated deoxyfluorination reactions as shown in Scheme 1a. However, no yields or NMR data were reported. The second example (Scheme 1b) was published by Fleet and co-workers, where deoxyfluorination of 16 using XtalFluor-M/Et ${ }_{3} \mathrm{~N} \cdot 3 \mathrm{HF}$ did not lead to the desired difluorinated azetidine derivative (not shown), but instead yielded the ring-expanded product $17 .^{37}$ Deprotection of $\mathbf{1 7}$ led to $(3 R, 4 R)$-3,4-difluoroproline 6. Hence, in both cases, the $\mathrm{C}-\mathrm{F}$ bond introduction was achieved in sequential fashion. Finally, our group recently reported a stereoselective synthesis of Boc-protected (3S,4R)-3,4-difluoroproline (N-Boc)-4, which featured a direct bis-deoxyfluorination step (Scheme 1c). ${ }^{34}(3 R, 4 S)-3,4-D i h y d r o x y p r o l i n e ~ 19 a$, obtained by selective dihydroxylation of the corresponding 3,4dehydroproline, was treated with nonafluorobutanesulfonyl fluoride (NfF) in combination with tetrabutylammonium triphenyldifluorosilicate (TBAT) to yield 20a as the only observed 3,4-difluoroproline.

In this work, we describe in detail the synthesis of the yet unreported (3S,4S)-3,4-difluoroproline $\mathbf{5}$ and a novel, more concise route for $(3 R, 4 R)-3,4$-difluoroproline 6 , both as their $N$-Boc derivatives, and as their $N$-acetylated methyl esters 21 and 22 (Scheme 2). Following our earlier communication, the development of the synthesis of N-Boc-4, including further optimization efforts of the bis-deoxyfluorination step as well as a direct synthesis of (N-Fmoc)-4, is described. The ring pucker analyses, prolyl bond cis/trans ratios, and isomerization kinetics of 21 and 22 are described and compared to those of unmodified proline and the four known monofluorinated proline derivatives. Since $\mathbf{5 / 2 1}$ can be regarded as a combination of (4R)-FPro and (3R)-FPro, both known to be biased to the $\mathrm{C}^{\gamma}$ exo pucker and trans peptide bond configuration relative to proline, ${ }^{14}$ it was anticipated that $\mathbf{5}$ / 21 will display a conformational bias in the same direction. Similarly, $\mathbf{6 / 2 2}$ was expected to have a larger proportion of the $\mathrm{C}^{\gamma}$ endo pucker and of the cis peptide bond configuration relative to proline, as it is a combination of (4S)-FPro and (3S)-FPro.

\section{RESULTS AND DISCUSSION}

Retrosynthetic Analysis. Our retrosynthetic analysis of 3,4-difluoroprolines is outlined in Scheme 2. Functional group interconversion to epoxides $9 \mathbf{a} / \mathbf{b}$ and $\mathbf{1 0 a} / \mathbf{b}$, as in the Novartis work, appeared attractive, as it would allow direct epoxide opening with fluoride followed by deoxyfluorination of the resulting fluorohydrin. Alternatively, diol 19a/c provided an 
Scheme 1. Precedence for the Synthesis of 3,4-Difluoroprolines

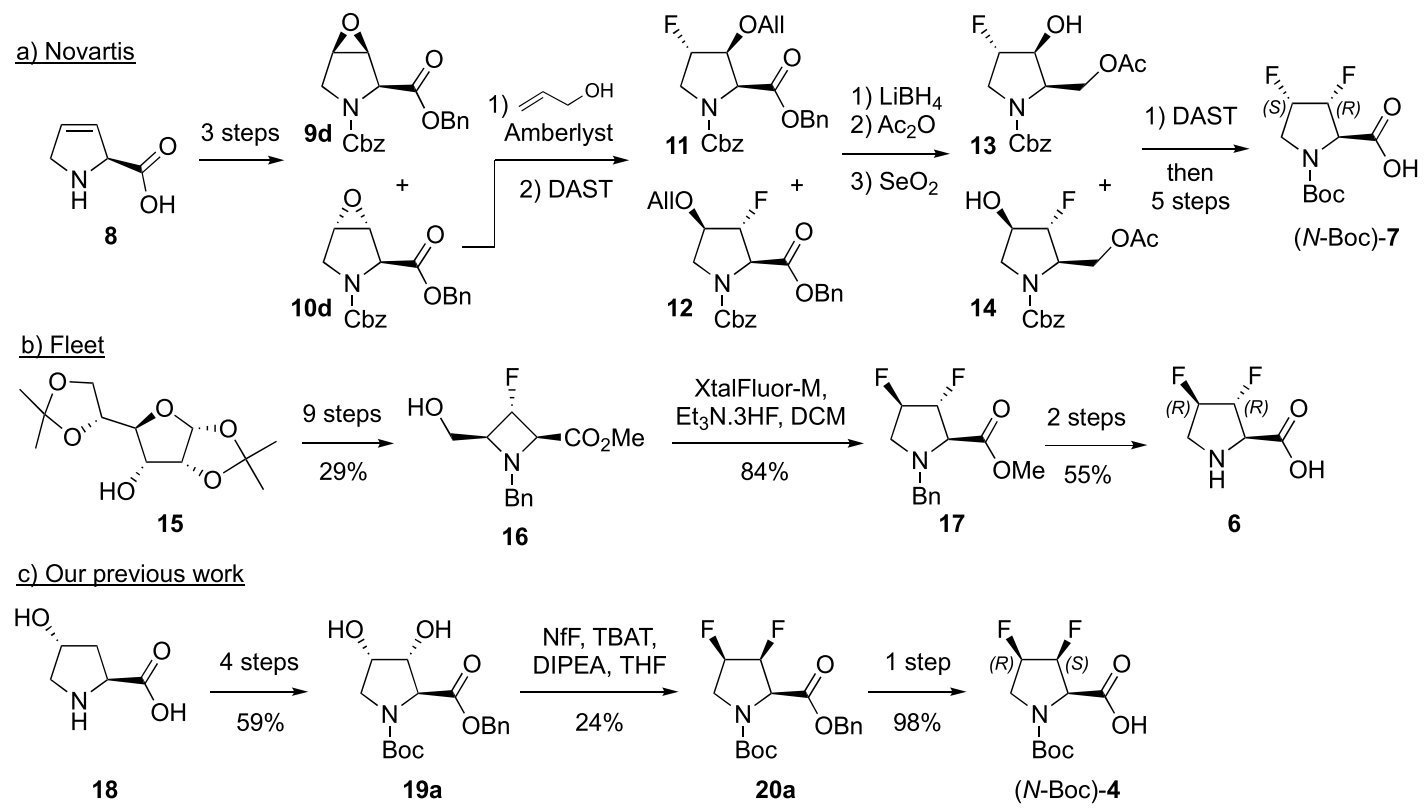

Scheme 2. Retrosynthetic Analysis

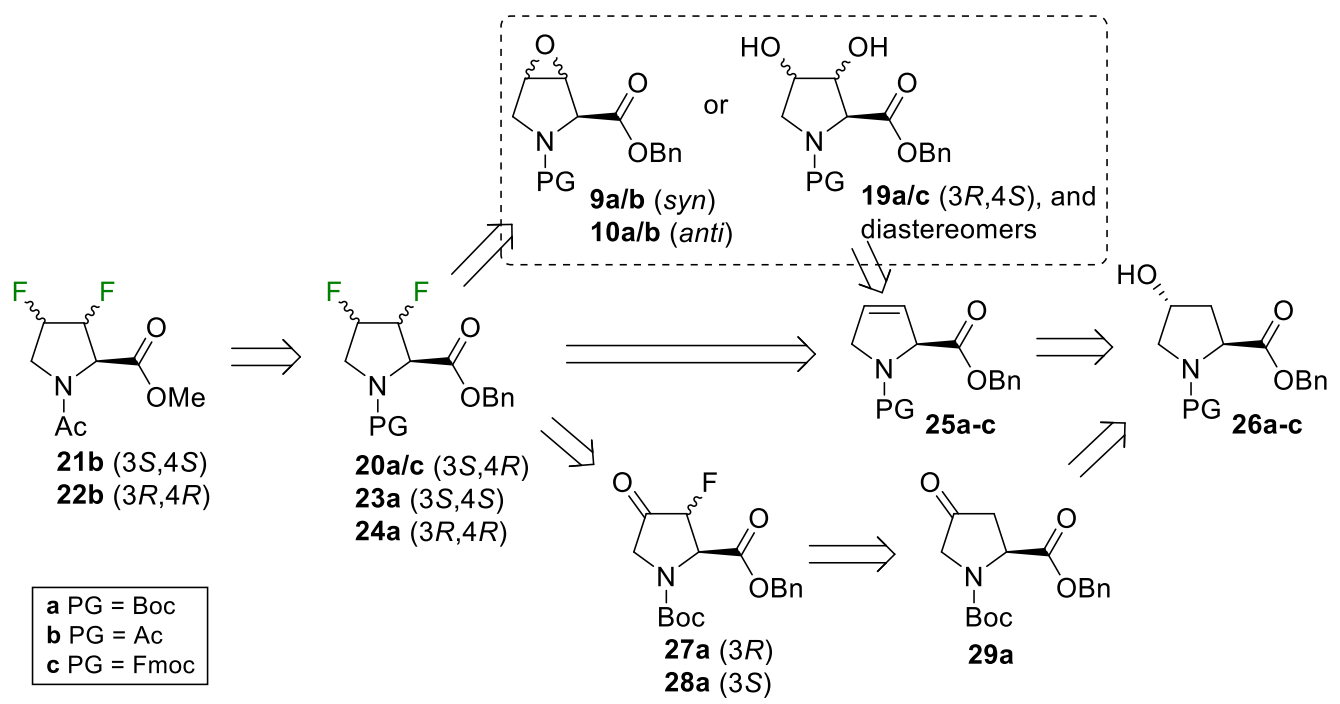

approach for 3,4-difluorination, with an excellent precedence available from the Marson group, who obtained trans-3,4difluoropyrrolidine from trans-3,4-dihydroxypyrrolidine via the corresponding triflates. ${ }^{38}$ While the epoxides and diols would be accessed from 3,4-dehydroderivatives $25 \mathbf{a}-\mathbf{c}$, direct functionalization of $\mathbf{2 5 a}-\mathbf{c}$ such as vicinal difluorination or a halofluorination/fluoride halide displacement could also lead to the desired 3,4-difluoroprolines. 3,4-Dehydroproline is a commercially available (expensive) building block but can also be obtained by a well-described elimination process involving 26a-c starting from cheap (4R)-4-hydroxyproline. Finally, an electrophilic fluorination approach as recently described by Ciulli et al. $^{39}$ leading to $\mathbf{2 7 a}$ /28a was also envisaged. With facile deprotection and versatility in mind, a benzyl ester in combination with various amine protecting groups were used throughout our investigations.

3,4-Dehydroproline Synthesis. Initial efforts focused on achieving a large-scale synthesis of 3,4-dehydroproline 25 . Following a literature protocol, conversion of protected (4R)- 4-hydroxyproline $26 a$ to the corresponding iodide, via a Mitsunobu reaction, ${ }^{40}$ followed by DBU-promoted HI elimination, gave a $\pm 5: 1$ mixture of alkene regioisomers, from which the desired alkene 25 a could be isolated in an excellent combined $76 \%$ yield (not shown), with $16 \%$ of the undesired 4,5-alkene 31a. While this elimination reaction gave 25a as a pure enantiomer ( $>97 \%$ ee, see Supporting Information), the separation of the alkene isomers was cumbersome. Moreover, it was found that conversion of $\mathbf{2 6 b}$ to the corresponding 4-OMs derivative $30 \mathrm{~b}$ (Scheme 3), followed by elimination using the same base, led to a mixture $( \pm 2: 1$ ratio $)$ of racemic alkene $25 \mathbf{b}$ and partially racemized 31b. A 89:11 ratio of amide rotamers of $\mathbf{3 1} \mathbf{b}$ was observed in the NMR spectra, with NOESY analysis showing the trans isomer being the major rotamer (see Supporting Information). Pleasingly, a one-pot Grieco elimination sequence ${ }^{41}$ directly starting from 26a gave enantiopure 25a as the major regioisomer with an increased regioselectivity ( $>10: 1$ ratio), and with a negligible degree of racemization. The smaller 
Scheme 3. Synthesis of Protected 3,4-Dehydroprolines $25 a-c$

$\mathrm{X}$

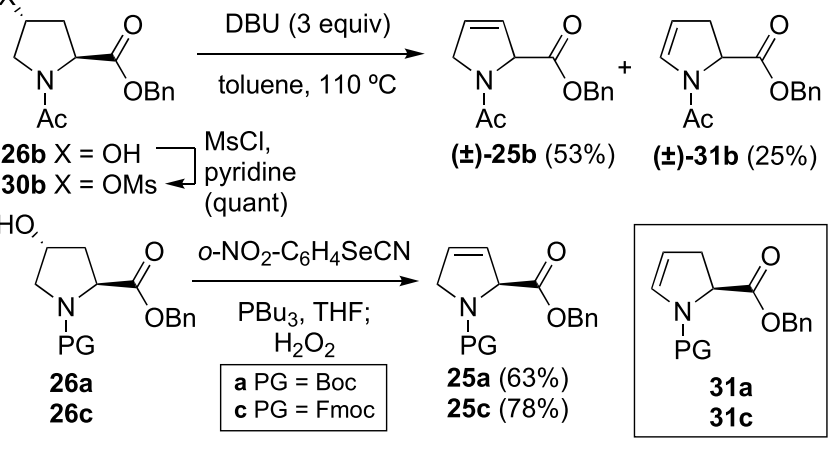

amount of 31a facilitated purification considerably. Furthermore, in contrast to base-mediated elimination reactions, it was found that direct Grieco elimination of (4R)-4-hydroxyproline could also be performed with an $\mathrm{N}$-Fmoc-protecting group (26c) in a very good yield. This procedure is an improvement over the previously reported two-step elimination via $4-\mathrm{SePh}$ intermediates, which are typically prepared from the corresponding 4-OMs or 4-OTs derivatives. ${ }^{42-46}$

Vicinal Difluorination and Halofluorination. Direct vicinal difluorination of alkene 25a was attempted using recent methods developed by Gilmour and Jacobsen, both based on the in situ generation of a hypervalent iodoarene difluoride. ${ }^{47,48}$ Unfortunately, both methods were unsuccessful and only led to recovered starting material (not shown). Subsequent attempts to effect halofluorination on 25a using different combinations of NBS, NCS, or NIS with either HF. pyridine or $\mathrm{Et}_{3} \mathrm{~N} \cdot \mathrm{HF}$ were unsuccessful as well, and this line of research was abandoned.

Epoxide-Based Strategy. Epoxidation of 3,4-dehydroproline derivatives is known, but not with the Boc/Bn- or Ac/Bnprotecting group combinations. Following protocol, treatment of $25 a / b$ with mCPBA led to a mixture of epoxides $9 a / b$ and $10 a / b$ in good yields with the trans isomer $10 a / b$ isolated as the major isomer after chromatography (Scheme 4). While

Scheme 4. Synthesis of Protected 3,4-Epoxyprolines

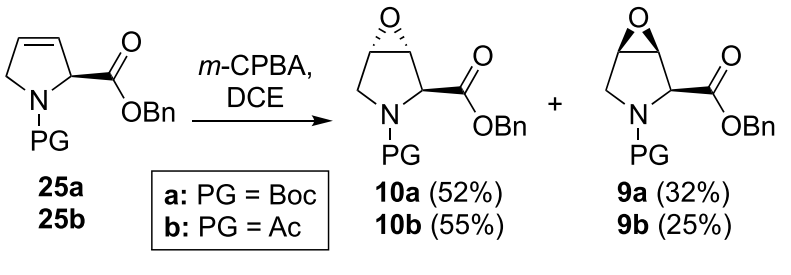

determination of the epoxide stereochemistry was achieved by ${ }^{1} \mathrm{H}$ NMR analysis as reported by Robinson et al. on $\mathrm{N}$-Cbz-3,4epoxyproline benzyl esters (Supporting Information), ${ }^{49}$ unambiguous conformation of the stereochemistry was obtained by X-ray crystallographic analysis of $\mathbf{9 b}$ (Supporting Information).

First, epoxide 10a was investigated as a substrate for direct fluoride opening with HF reagents (Table 1). Reaction with $\mathrm{Et}_{3} \mathrm{~N} \cdot 3 \mathrm{HF}$ in dichloroethane (DCE) at $80{ }^{\circ} \mathrm{C}$ for 3 days resulted in a complex mixture of chlorinated and fluorinated products $( \pm 15 \%)$, alongside $68 \%$ of the recovered starting material (not shown), but conducting the reaction neat with increasing the reaction temperature to $130{ }^{\circ} \mathrm{C}$ (entry 1) induced deprotection and aromatization, leading to pyrrole 35 in a quantitative yield. Due to its low reactivity, the use of $\mathrm{Et}_{3} \mathrm{~N} \cdot 3 \mathrm{HF}$ is often characterized by long reaction times and high reaction temperatures, which can be alleviated by microwave irradiation. ${ }^{50}$ However, with a short reaction time, no product was observed and increasing the reaction time and temperature led to pyrrole 35 (entries 2-4). With the more reactive DMPU.HF, ${ }^{51}$ reaction of $\mathbf{1 0 b}$ did lead to fluorohydrin $33 \mathbf{b}$ in a $15 \%$ yield, together with $30 \%$ of the recovered starting material (entry 5). Unfortunately, raising the reaction time and temperature did not improve the yield (entry 6). These reactions suffered from gel formation, which impeded the isolation of the products. The use of hexafluoroisopropanol (HFIP) as an additive successfully disrupted gel formation, but no fluorination was observed (not shown). Next, epoxide opening was attempted with $\mathrm{Bu}_{4} \mathrm{NH}_{2} \mathrm{~F}_{3}$. Unexpectedly, the reaction at reflux in DCE yielded chlorohydrin 32a (entry 7). Presumably, decomposition of the solvent under these conditions must have released chloride ions, which subsequently opened the epoxide. In toluene, $\mathrm{Bu}_{4} \mathrm{NH}_{2} \mathrm{~F}_{3}$ was found to be too basic, with fluoride causing $\mathrm{H}^{\alpha}$ deprotonation, leading to the formation of allylic alcohol 34a (entry 8). This was also the major pathway upon reaction with TBAF in $t$ $\mathrm{BuOH}$ (entry 9). Interestingly, in contrast to the 4,5-dehydro isomer $31 \mathbf{b}$, the ${ }^{13} \mathrm{C}$ and ${ }^{1} \mathrm{H}$ NMR spectra of $34 \mathrm{~b}$ only showed a single set of resonances, which could indicate the presence of a single rotamer. The NOESY NMR spectrum of $34 \mathbf{b}$ is consistent with the trans rotamer (Supporting Information). With $\mathrm{KHF}_{2}$ in ethylene glycol at $150{ }^{\circ} \mathrm{C}$ (entry 10), aromatization and transesterification was observed, yielding 36.

With direct fluoride opening being unsuccessful, it was then attempted to perform fluorination after prior epoxide opening with different nucleophiles (Scheme 5). Precedence for opening of proline epoxides includes reaction with $\mathrm{MgI}_{2}$ $(78 \%)^{52}$ and 2-chloro-3-ethylbenzoxazolium tetrafluoroborate $(32 \%),{ }^{53}$ both exclusively at the 4-position.

Starting from $10 b$, regioselective opening with $\mathrm{HCl}$, HOTs, and $\mathrm{HBr}$ (or $\mathrm{MgBr}_{2}$ ) led to the corresponding 4-substituted 3hydroxyprolines $32 \mathrm{~b}, 37 \mathrm{~b}$, and $38 \mathrm{~b}$ in excellent yields. However, subsequent DAST-mediated deoxyfluorination reactions mostly led to aromatization: for the chlorohydrin $\mathbf{3 2 b}$, pyrrole $39 \mathrm{~b}$ was the only product isolated, while, with the $\beta$ hydroxy tosylate $37 \mathbf{b}$, a low yield of the desired 3-fluorinated product $40 \mathrm{~b}$ was obtained, alongside $62 \%$ of pyrrole $39 \mathrm{~b}$. Tentative assignment of the expected stereochemistry of $40 \mathrm{~b}$ at $\mathrm{C}_{\beta}$ was based on the observed coupling constant of $5 \mathrm{~Hz}$ between $\mathrm{H}_{\alpha}$ and $\mathrm{H}_{\beta}$. Attempts to achieve fluorination at the 4position in the presence of the $3-\mathrm{OH}$ group by bromide or tosylate displacement with TBAF-t-BuOH were also unsuccessful. Starting from $37 \mathrm{~b}$, a mixture of allylic alcohol $34 \mathrm{~b}$ and epoxide $10 \mathrm{~b}$ was obtained. Despite the reduced basicity due to hydrogen bonding with $t-\mathrm{BuOH}$, fluoride must have deprotonated the alcohol group of $37 \mathbf{b}$ causing epoxide formation, followed by $\mathrm{H}_{\alpha}$ deprotonation, resulting in epoxide opening to give $\mathbf{3 4 b}$. Using bromohydrin $38 \mathrm{~b}$, the same allylic alcohol 34b was the only product isolated. Interestingly, treating $\mathbf{3 8 b}$ with $\mathrm{AgF}$ in nitromethane only led to epoxide formation in a quantitative yield.

At this point, the epoxide-based strategy was abandoned, and attention shifted to fluorine introduction via a vicinal diol group.

Direct Bis-deoxyfluorination Approach. Dihydroxylation of Cbz-protected 3,4-dehydroproline 25d with $\mathrm{OsO}_{4}$ has 
Table 1. Conditions Investigated for the Direct Fluoride Opening of Epoxides 10a and 10b
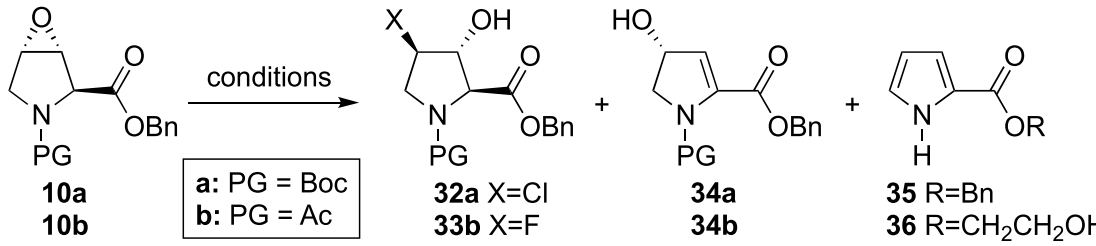

\begin{tabular}{|c|c|c|c|c|c|}
\hline entry & PG & conditions & $T\left({ }^{\circ} \mathrm{C}\right)$ & $t(\mathrm{~h})$ & product (\%) \\
\hline 1 & Boc & $\mathrm{Et}_{3} \mathrm{~N} \cdot 3 \mathrm{HF}$ (neat) & 130 & 72 & 35 (quant) $^{b}$ \\
\hline 2 & Boc & $\mathrm{Et}_{3} \mathrm{~N} \cdot 3 \mathrm{HF} / \mathrm{THF}(2: 1), \mathrm{MW}^{c}$ & 100 & 0.08 & 10a $(86), 35(7)^{b}$ \\
\hline 3 & Boc & $\mathrm{Et}_{3} \mathrm{~N} \cdot 3 \mathrm{HF} / \mathrm{THF}(2: 1), \mathrm{MW}^{c}$ & 100 & 0.33 & 10a $(72), 35(28)^{b}$ \\
\hline 4 & Boc & $\mathrm{Et}_{3} \mathrm{~N} \cdot 3 \mathrm{HF} / \mathrm{THF}(3: 1), \mathrm{MW}^{c}$ & 130 & 0.66 & $35{\text { (quant })^{b}}^{b}$ \\
\hline 5 & Ac & DMPU·HF, DCM & $\mathrm{rt} \rightarrow 50$ & 43 & 10b (30), 33b (15) \\
\hline 6 & Ac & DMPU·HF, DCE & 60 & 72 & $10 \mathbf{b}(25), 33 \mathbf{b}(5)$ \\
\hline 7 & Boc & $\mathrm{Bu}_{4} \mathrm{NH}_{2} \mathrm{~F}_{3}, \mathrm{DCE}$ & 120 & 25 & $32 \mathrm{a}(74)$ \\
\hline 8 & Boc & $\mathrm{Bu}_{4} \mathrm{NH}_{2} \mathrm{~F}_{3}$, toluene & 120 & 24 & $34 a(56)$ \\
\hline 9 & Ac & $\mathrm{TBAF}, t-\mathrm{BuOH}$ & 70 & 4 & 34b (30), $35(13)$ \\
\hline 10 & Boc & $\mathrm{KHF}_{2}$, ethylene glycol & 150 & 22 & $36(59), 35(2)$ \\
\hline
\end{tabular}

${ }^{a}$ Severe gel formation. ${ }^{b}$ Calculated yields based on ${ }^{1} \mathrm{H}$ NMR analysis of the crude reaction mixture. ${ }^{c}$ Microwave irradiation.

Scheme 5. Epoxide Opening with Other Nucleophiles and Subsequent Fluorination Attempts

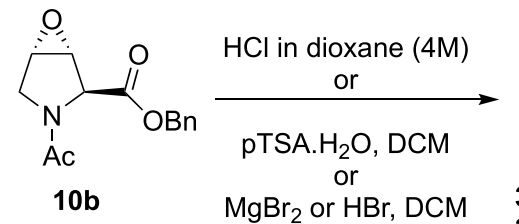<smiles>[X]C1CN(C(C)C)C(C(=O)OCc2ccccc2)[C@H]1O</smiles>

32b X $=\mathrm{Cl}(95 \%)$ $37 \mathbf{b} \times=$ OTs $(73 \%)$ 38b $X=\operatorname{Br}(88 \%, 95 \%)$

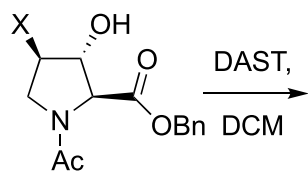

$32 \mathrm{~b} X=\mathrm{Cl}$

$37 b X=$ OTs

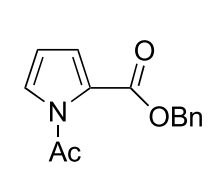

$39 \mathrm{~b}$

$(40 \%$, from $32 \mathrm{~b})$ $(62 \%$, from $37 b)$

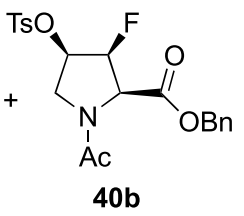

$(6 \%$, from $37 b)$

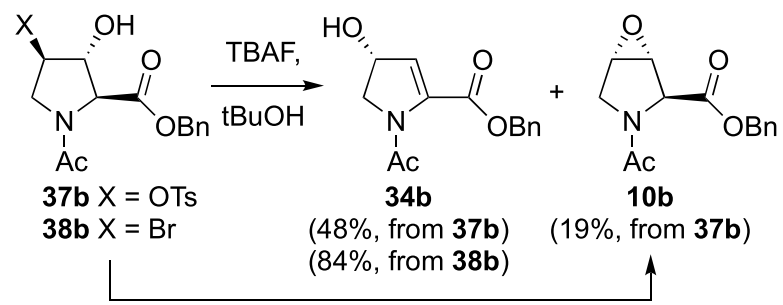

$\mathrm{AgF}, \mathrm{CH}_{3} \mathrm{NO}_{2}$ (quant)

been reported to be high-yielding and very stereoselective, leading to the 2,3-trans-2,4-trans-diol 19d as the major isomer. $^{44,45,54}$ A similar result was observed when these conditions were applied to 25a (Table 2, entry 1). Interestingly, starting from the Boc-protected 25a with the osmate ester (entry 2), no all-cis-diol 41a was observed. ${ }^{34}$ As both diastereomeric cis diols were desired, attempts to promote the formation of all-cis-diol 41a using Sharpless asymmetric dihydroxylation $^{55}$ conditions were carried out. However, reacting 25a with both $\mathrm{AD}$-mix- $\alpha$ and $\mathrm{AD}$-mix- $\beta$ only led to the formation of $19 \mathrm{a}$ in $82 \%$ and $66 \%$ yields, respectively (entries 3 and 4). Finally, dihydroxylation was also carried out on the Fmoc-protected alkene 25c using the osmate ester
Table 2. Dihydroxylation of 3,4-Dehydroproline 25a/c
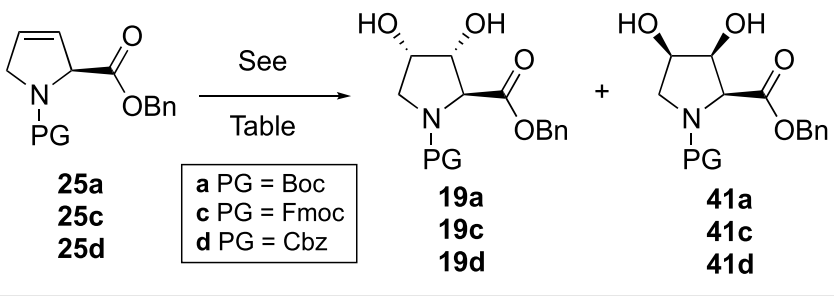

\begin{tabular}{cllc} 
entry & PG & \multicolumn{1}{c}{ conditions } & $\begin{array}{c}\text { yield 19a/c } \\
(\%)\end{array}$ \\
1 & $\mathrm{Boc}$ & $\mathrm{OsO}_{4}, \mathrm{NMO}, \mathrm{H}_{2} \mathrm{O} /$ dioxane $(1: 4)$ & 92 \\
2 & $\mathrm{Boc}$ & $\mathrm{K}_{2} \mathrm{OsO} 4 \cdot 2 \mathrm{H}_{2} \mathrm{O}, \mathrm{NMO}, \mathrm{H}_{2} \mathrm{O} /$ acetone $(1: 3)$ & 94 \\
3 & $\mathrm{Boc}$ & $\mathrm{AD}-\mathrm{mix} \alpha, t-\mathrm{BuOH} / \mathrm{H}_{2} \mathrm{O}(1: 1)$ & 82 \\
4 & $\mathrm{Boc}$ & $\mathrm{AD}-\mathrm{mix} \beta, t-\mathrm{BuOH} / \mathrm{H}_{2} \mathrm{O}(1: 1)$ & 66 \\
5 & Fmoc & $\mathrm{K}_{2} \mathrm{OsO}_{4} \cdot 2 \mathrm{H}_{2} \mathrm{O}, \mathrm{NMO}, \mathrm{H}_{2} \mathrm{O} /$ acetone $(1: 3)$ & 80 \\
\hline
\end{tabular}

conditions, also exclusively leading to N-Fmoc-protected 2,3trans-2,4-trans-diol 19c (entry 5).

Marson et al. previously demonstrated that, starting from a trans-3,4-ditriflate substituted pyrrolidine ring 43 (Scheme 6), vicinal difluorination with TBAF can yield the corresponding trans-3,4-difluoropyrrolidine 44 in a good yield, ${ }^{38,56}$ and this transformation has also been successful on the corresponding Cbz derivative. ${ }^{57}$ However, treatment of 3,4-dihydroxyproline 19a with triflic anhydride already resulted in the formation of pyrrole $39 \mathrm{a}$ in a $64 \%$ yield. Hence, reaction with nonafluorobutanesulfonyl fluoride $(\mathrm{NfF})^{58}$ in combination with tetrabutylammonium difluorotriphenylsilicate $(\mathrm{TBAT})^{59}$ was attempted, as this process generates sulfonates in the presence of fluoride. Pleasingly, this led to 20a as the only observed 3,4difluoroproline diastereoisomer ( ${ }^{19} \mathrm{~F}$ NMR analysis), with an enol sulfonate 46a as major byproduct along with its hydrolysis product, 3-oxoproline, as a minor, but persistent, impurity (not shown). Interestingly, no pyrrole side product was observed. While separation of all products was possible by HPLC, purification was considerably facilitated by subjecting the reaction mixture to $\mathrm{NaBH}_{4}$ in order to reduce the 3-oxoproline byproduct to the corresponding alcohol (not shown). The regiochemistry of enol sulfonate 46 a was established by means of a 2D HOESY NMR experiment. 
Scheme 6. Fluorination of Dihydroxyproline via Sulfonate Intermediates
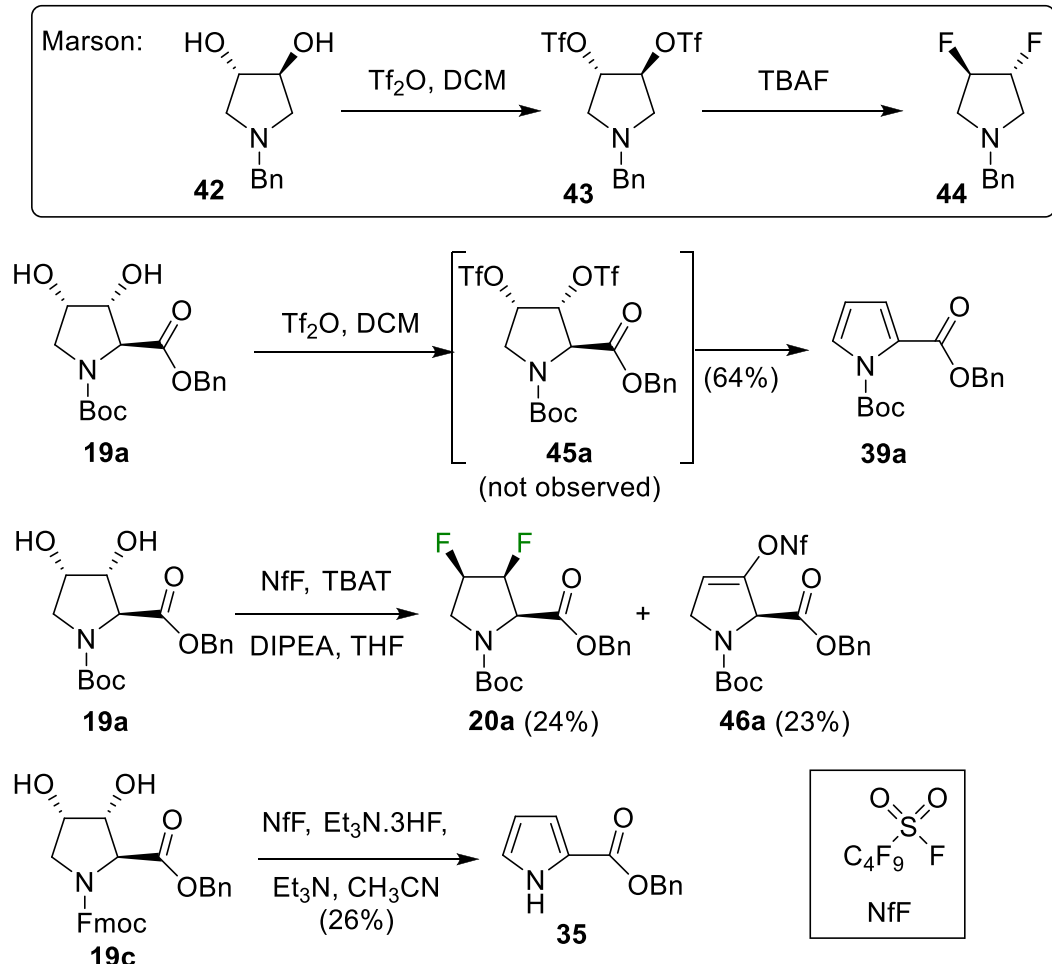

Scheme 7. Fluorination of Dihydroxyproline Using DAST

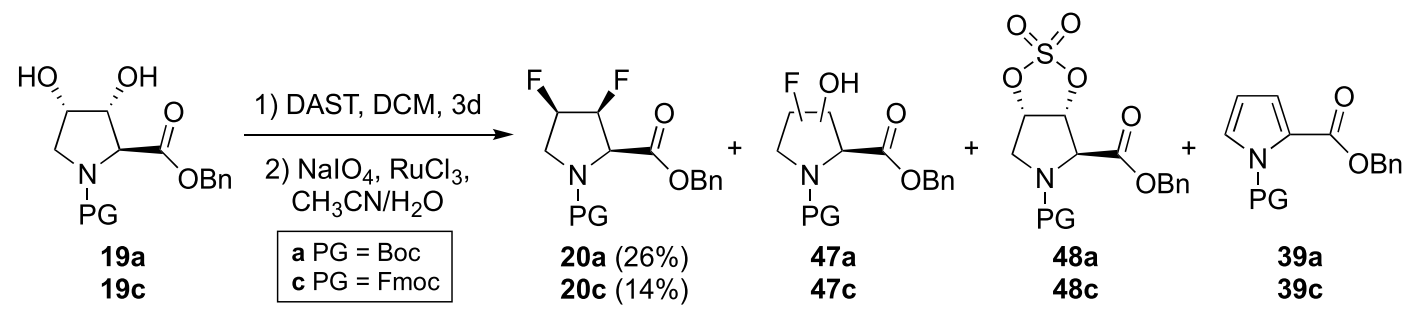

Scheme 8. Electrophilic Fluorination Route to 23a and 24a

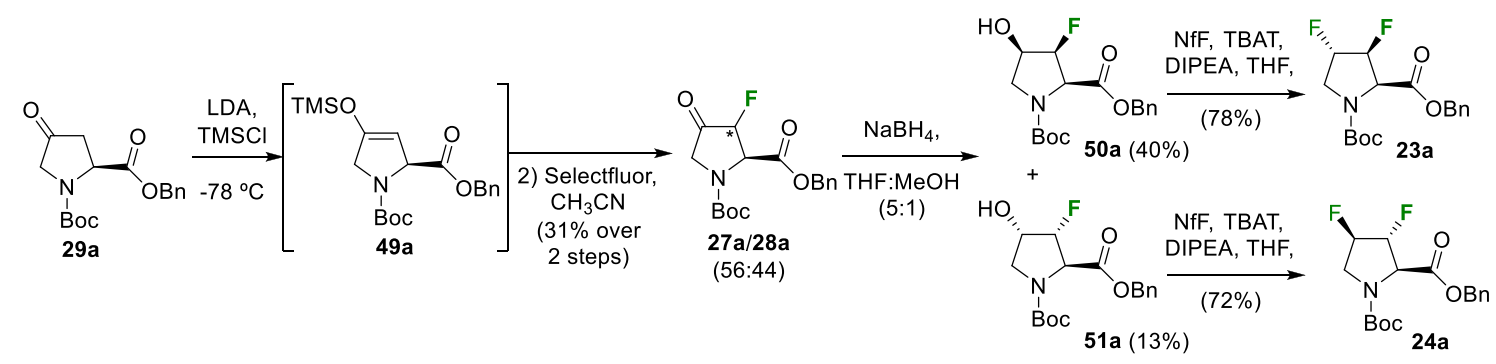

As a Fmoc-protecting group does not tolerate basic conditions, TBAT could not be used as a fluoride source for the $\mathrm{NfF}$ fluorination. Even when (diluted) $\mathrm{Et}_{3} \mathrm{~N} \cdot 3 \mathrm{HF} / \mathrm{Et}_{3} \mathrm{~N}$ was employed as a fluoride source, ${ }^{60}$ no difluorination was observed in the crude ${ }^{19} \mathrm{~F}$ NMR and pyrrole 35 was the only product obtained from the reaction.

The reaction of the 3,4-diols $19 a$ and $19 \mathrm{c}$ was also investigated with DAST (Scheme 7). With 19a, this led to a complex reaction mixture, in which the desired difluorinated 20a was clearly visible by ${ }^{19} \mathrm{~F}$ NMR analysis, next to two minor byproducts, which presumably were monofluorinated hydroxyfluoroprolines 47a. As the desired 20a coeluted with another byproduct, identified as the corresponding cyclic sulfite, the crude reaction mixture was subjected to typical oxidation conditions, leading to the formation of the cyclic sulfate $48 \mathrm{a}$. Isolation was now possible, leading to 20 a in a $26 \%$ yield. According to MS analysis, the sulfite oxidation was not accompanied by possible ${ }^{61}$ proline C5-oxidation to the corresponding lactam. Similarly, when this sequence was applied to the Fmoc-protected 19c, the desired 3,4difluoroproline 20c was also isolated, albeit in a reduced $14 \%$ yield.

Despite the low yield of this double deoxyfluorination process, the very short synthesis (only three steps from protected (4R)-hydroxyproline) was deemed an acceptable 
and practical synthesis, as gram-scale quantities of 20a could readily be obtained.

Electrophilic Fluorination Strategy. With no straightforward access to other 3,4-dihydroxyproline diastereoisomers as substrates for bis-deoxyfluorination, investigations turned toward an electrophilic fluorination approach. Barraclough et al. had demonstrated the regioselective conversion of a 4ketoproline derivative to the corresponding silyl enolether, ${ }^{62,63}$ which was used to stereoselectively introduce deuterium at C3. Hence, formation of the silyl enol ether 49a was achieved upon treatment of 29a, ${ }^{64-66}$ synthesized by Dess-Martin periodinane oxidation of 26a in $94 \%$ yield (not shown), with LDA and TMSCl, and subsequently fluorinated with SelectFluor (Scheme 8). In our hands, this transformation proved to be low-yielding and was found difficult to optimize, leading to a mixture of isomers $\mathbf{2 7 a / 2 8 a}$ in a maximum $31 \%$ yield. Reduction of the 4-keto group led to a mixture of two separable fluorohydrin isomers, 50a and 51a, in a moderate yield. In the course of the optimization process, Ciulli and coworkers reported the synthesis of $\mathbf{2 7 a / 2 8 a}$ in $50 \%$ yield using this procedure, and of 50a/51a in $58 \%$ and $30 \%$ yields, respectively. ${ }^{39}$ Interestingly, they also isolated a third diastereomer. Preliminary assignment of the stereochemistry at $\mathrm{C}_{\beta}$ was based on the observed coupling constant between $\mathrm{H}_{\alpha}$ and $\mathrm{H}_{\beta}$, which was $\sim 6 \mathrm{~Hz}$ for 50a and $\sim 2 \mathrm{~Hz}$ for 51a. This value for 50a is in line with the coupling constant observed between $\mathrm{H}_{\alpha}$ and $\mathrm{H}_{\beta}$ in 20a. In addition, for 50a, clear NOESY cross peaks were observed between $\mathrm{H}_{\alpha}$ and $\mathrm{H}_{\beta}$ and between $\mathrm{H}_{\beta}$ and $\mathrm{H}_{\gamma}$, suggesting all protons are on the same $\alpha$-face of the pyrrolidine ring. This assignment was in agreement with the Ciulli work. ${ }^{39}$

Deoxyfluorination of both 50a and 51a was achieved in a very good yield by treatment with the NfF and TBAT reagent combination. The stereochemistry of $\mathbf{2 4 a}$ was unambiguously assigned by means of X-ray analysis (Figure 3).

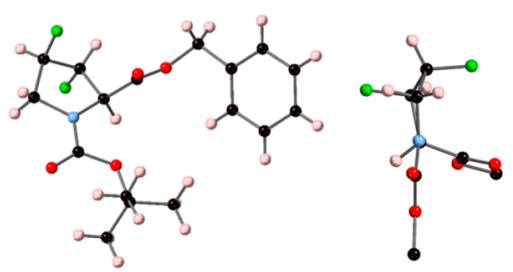

Figure 3. X-ray structure of $(3 R, 4 R)$-3,4-difluoroproline 24a. Thermal ellipsoids drawn at the $50 \%$ probability level.

With the new 3,4-difluoroproline derivatives 23a and 24a in hand, conversion to the required $N$-acetyl methyl ester derivatives $\mathbf{2 1}$ and $\mathbf{2 2}$ was carried out to allow conformational studies, including comparison with other, known, $\mathrm{N}$-acetylated fluoroproline methyl esters. ${ }^{10,13,17,67}$ Hence (Scheme 9), the benzyl-protecting group was removed by hydrogenolysis, and the $\mathrm{N}$-Boc group by treatment with methanolic $\mathrm{HCl}$. These conditions also simultaneously effected methyl ester formation. Finally, the amine groups were converted to their corresponding $\mathrm{N}$-acetyl derivatives $\mathbf{2 1}$ and $\mathbf{2 2}$.

It was possible to obtain single crystals of 21, and crystallographic analysis (Figure 4) provided unambiguous proof of its relative stereochemistry.
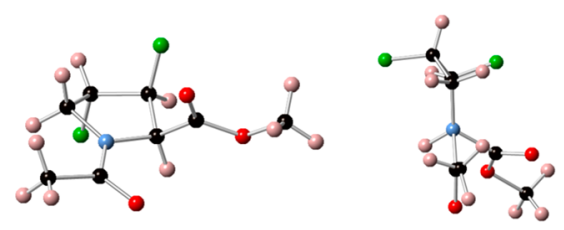

Figure 4. Crystal structure of (3S,4S)-3,4-difluoroproline 21. Thermal ellipsoids drawn at the $50 \%$ probability level.

Conformational and Kinetic Analyses. The experimental cis/trans ratios in chloroform and water, the experimental cis/trans isomerization rate constants in water, and the DFTcalculated pucker preferences for the $N$-Ac-X-OMe model compounds of proline, the $(3 S, 4 R)-,(3 R, 4 R)-$, and $(3 S, 4 S)$ 3,4 -difluorinated prolines and their monofluorinated progenitors are reported in Table 3. The entries are organized according to pucker preference. The data for the $(3 S, 4 R)$ variant $\mathbf{5 6}$ has been reported and discussed previously, ${ }^{34}$ but are included in Table 3 for the sake of completeness. In the following discussion, the term "bias" assumes the conformational preference of the nonfluorinated $\mathrm{N}$-acetyl proline methyl ester as a reference.

The amide cis/trans ratios in both chloroform and water of the 3,4-difluorinated proline $\mathbf{2 1}$ are very similar to those of each of their monofluorinated progenitors 52 and 53. For 22, the ratios are closer to those of $(4 S)$-fluoroproline $\mathbf{5 4}$ than the (3S)-derivative $\mathbf{5 5}$.

The cis/trans isomerization rates (represented here by $k_{e x}=$ $\left.k_{\text {cis/trans }}+k_{\text {trans/cis }}\right)$ typically increase with an increasing number of fluorine substitutions, mostly due to the electron-withdrawing effect of the fluorine atoms decreasing the double bond character of the amide bond. ${ }^{11}$ As expected, both the $(3 S, 4 S)$ - and $(3 R, 4 R)$-difluorinated variants, 21 and 22, indeed show higher isomerization rates than their monofluorinated progenitors. Interestingly, the $(3 R)$-variant $\mathbf{5 3}$ has a markedly higher isomerization rate than all other monofluorinated prolines, $^{29}$ and even exchanging faster than the $(3 R, 4 R)$ difluorinated variant 22 . This remarkable acceleration by fluorination at the 3-position with this stereochemistry is retained when combined with fluorination at the 4-position, resulting in even higher isomerization rates for the $(3 S, 4 S)$ variant $\mathbf{2 1}$. The isomerization rate for $\mathbf{2 1}$ is also much higher than that of the previously described $(3 S, 4 R)$ - and $(4,4)$ difluorinated variants. ${ }^{34}$

Scheme 9. Synthesis of N-Acetyl Methyl Ester Derivatives 21 and 22

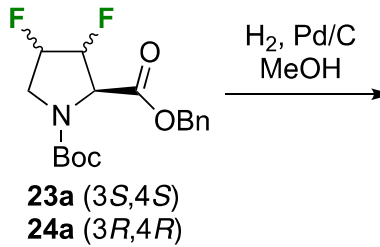

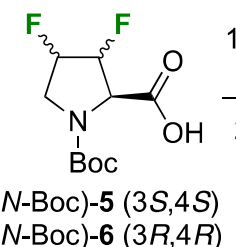

$(N-B o c)-6(3 R, 4 R)$

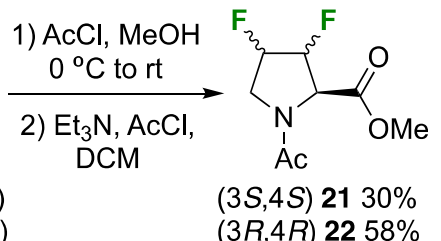

$(3 R, 4 R) 2258 \%$ 
Table 3. Experimental trans/cis Ratios and Amide Isomerization Rates and Calculated Pucker Ratios
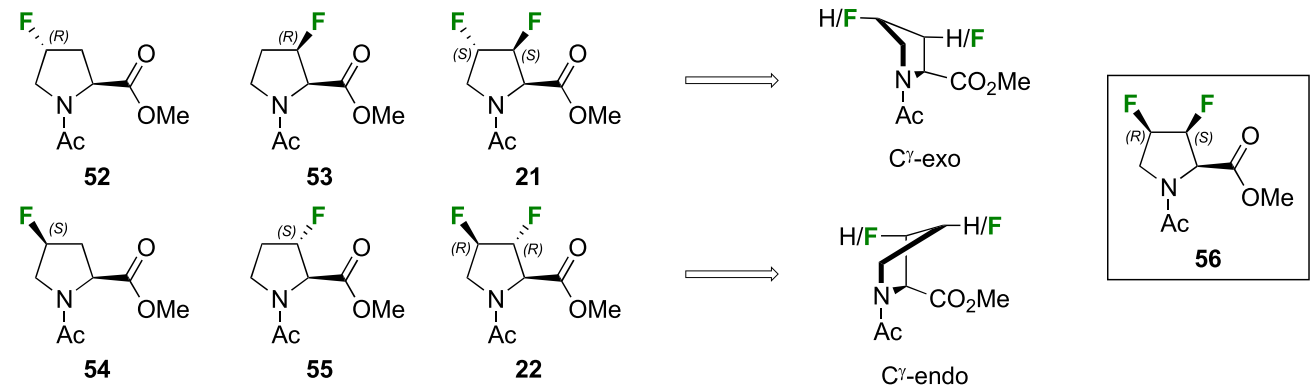

\begin{tabular}{|c|c|c|c|c|c|c|c|c|c|}
\hline \multirow[b]{3}{*}{ compound } & \multirow{2}{*}{\multicolumn{2}{|c|}{$K_{\text {cis/trans }}(\exp )\left(25^{\circ} \mathrm{C}\right)$}} & \multirow{2}{*}{\multicolumn{3}{|c|}{ cis/trans kinetics, $35{ }^{\circ} \mathrm{C}\left(\mathrm{s}^{-1}\right)(\exp )$}} & \multicolumn{4}{|c|}{$\mathrm{C}^{\gamma}$ endo $/ \mathrm{C}^{\gamma}$ exo ${ }^{i}$ (DFT) } \\
\hline & & & & & & \multicolumn{2}{|c|}{$\mathrm{CHCl}_{3}$} & \multicolumn{2}{|c|}{$\mathrm{H}_{2} \mathrm{O}$} \\
\hline & $\mathrm{CDCl}_{3}$ & $\mathrm{D}_{2} \mathrm{O}$ & $k_{\text {cis/trans }}$ & $k_{\text {trans } / \text { cis }}$ & $k_{e x}^{d}$ & trans & cis & trans & cis \\
\hline Ac-Pro-OMe & $3.85^{b}$ & $4.62^{b}$ & $0.031^{e}$ & $0.007^{e}$ & 0.038 & $81: 19$ & $90: 10$ & $66: 34$ & $82: 18$ \\
\hline$(3 S, 4 R)-56$ & 3.72 & 5.00 & $0.119 \pm 0.009^{f}$ & $0.025 \pm 0.002^{f}$ & $0.144 \pm 0.011$ & $41: 59$ & $78: 22$ & $56: 44$ & $90: 10$ \\
\hline$(3 R)-53$ & 5.08 & $8.31^{c}$ & $0.141 \pm 0.021^{f g}$ & $0.019 \pm 0.003^{f g}$ & $0.159 \pm 0.024$ & $24: 76$ & $16: 84$ & $15: 85$ & $44: 56$ \\
\hline$(4 R)-52$ & $4.26^{b}$ & $6.74^{b}$ & $0.064^{e}$ & $0.010^{e}$ & 0.074 & $11: 89$ & $28: 72$ & $7: 93$ & $17: 83$ \\
\hline$(3 S, 4 S)-21^{a}$ & 4.32 & 7.23 & $0.210 \pm 0.005^{f}$ & $0.031 \pm 0.001^{f}$ & $0.242 \pm 0.006$ & $19: 81$ & $11: 89$ & $20: 80$ & $9: 91$ \\
\hline$(3 S)-55$ & 4.19 & $4.31^{c}$ & $0.030 \pm 0.004^{f, h}$ & $0.009 \pm 0.001^{f, h}$ & $0.038 \pm 0.005$ & $98: 2$ & $98: 2$ & $97: 3$ & 99:1 \\
\hline$(4 S)-54$ & $1.64^{b}$ & $2.49^{b}$ & $0.037^{e}$ & $0.015^{e}$ & 0.052 & $97: 3$ & $99: 1$ & $99: 1$ & $99.5: 0.5$ \\
\hline$(3 R, 4 R)-\mathbf{2 2}^{a}$ & 1.98 & 2.79 & $0.065 \pm 0.009^{f}$ & $0.024 \pm 0.003^{f}$ & $0.090 \pm 0.013$ & $97: 3$ & $99: 1$ & $99: 1$ & 99:1 \\
\hline
\end{tabular}

${ }^{a}$ Note that CIP prioritization changes with introduction of the second fluorine atom, so that $\mathbf{2 1}$ must be compared with $\mathbf{5 2}$ and $\mathbf{5 3}$, and $\mathbf{2 2}$ with $\mathbf{5 4}$ and 55. ${ }^{b}$ In good agreement with reported ratios by Siebler et al. ${ }^{10} \mathrm{In}$ good agreement with reported ratios by Kim et al. ${ }^{68} d_{k_{e x}}$ is defined as $k_{e x}=$ $k_{\text {cis/trans }}+k_{\text {trans/cis. }}{ }^{e}$ Calculated value based on Renner et al. ${ }^{13}{ }^{f}$ Experimental NMR value obtained using a similar procedure as Renner et al. ${ }^{13}$ ${ }^{g}$ Corresponding values reported by Thomas et al. at $37{ }^{\circ} \mathrm{C}$ using an alternative experimental procedure: $0.229 \mathrm{~s}^{-1}$ and $0.028 \mathrm{~s}^{-1} .{ }^{29} \mathrm{Corresponding}$ values reported by Thomas et al. at $37{ }^{\circ} \mathrm{C}$ using an alternative experimental procedure: $0.065 \mathrm{~s}^{-1}$ and $0.016 \mathrm{~s}^{-1}$. ${ }^{2}{ }^{i} \mathrm{DFT}$ values, using the M06 functional with cc-pVDZ basis set and $\mathrm{CHCl}_{3}$ or water SMD implicit solvent models.

Table 4. Comparison of the Relevant Coupling Constants

\begin{tabular}{|c|c|c|c|c|c|c|c|c|c|}
\hline \multirow[b]{3}{*}{ compound } & \multirow[b]{3}{*}{ bias $^{b}$} & \multicolumn{4}{|c|}{${ }^{3} J_{\mathrm{H}_{\alpha} \mathrm{F}_{\beta}}(\mathrm{Hz})^{a}$} & \multicolumn{4}{|c|}{${ }^{3} J_{\mathrm{H}_{\alpha} \mathrm{H}_{\beta}}(\mathrm{Hz})^{a}$} \\
\hline & & \multicolumn{2}{|c|}{ cis amide } & \multicolumn{2}{|c|}{ trans amide } & \multicolumn{2}{|c|}{ cis amide } & \multicolumn{2}{|c|}{ trans amide } \\
\hline & & cisoid $^{c}$ & transoid $^{c}$ & cisoid & transoid & cisoid & transoid & cisoid & transoid \\
\hline Ac-Pro-OMe & & $\mathrm{n} / \mathrm{a}$ & $\mathrm{n} / \mathrm{a}$ & $\mathrm{n} / \mathrm{a}$ & $\mathrm{n} / \mathrm{a}$ & 8.9 & 2.6 & 8.8 & 4.7 \\
\hline$(3 S, 4 R)-56$ & & $\mathrm{n} / \mathrm{a}$ & 7.4 & $\mathrm{n} / \mathrm{a}$ & 13.7 & 7.9 & $\mathrm{n} / \mathrm{a}$ & 7.3 & $\mathrm{n} / \mathrm{a}$ \\
\hline$(3 R)-53$ & exo & $\mathrm{n} / \mathrm{a}$ & 25.9 & $\mathrm{n} / \mathrm{a}$ & 28.2 & 5.1 & $\mathrm{n} / \mathrm{a}$ & 4.8 & $\mathrm{n} / \mathrm{a}$ \\
\hline$(4 R)-52$ & exo & $\mathrm{n} / \mathrm{a}$ & $\mathrm{n} / \mathrm{a}$ & $\mathrm{n} / \mathrm{a}$ & $\mathrm{n} / \mathrm{a}$ & 8.7 & 8.2 & 7.8 & 10.1 \\
\hline$(3 S, 4 S)-\mathbf{2 1}$ & exo & $\mathrm{n} / \mathrm{a}$ & 27.3 & $\mathrm{n} / \mathrm{a}$ & 29.8 & 5.2 & $\mathrm{n} / \mathrm{a}$ & 5.0 & $\mathrm{n} / \mathrm{a}$ \\
\hline$(3 S)-55$ & endo & 19.8 & $\mathrm{n} / \mathrm{a}$ & 13.7 & $\mathrm{n} / \mathrm{a}$ & $\mathrm{n} / \mathrm{a}$ & $<1.0^{d}$ & $\mathrm{n} / \mathrm{a}$ & 1.0 \\
\hline$(4 S)-54$ & endo & $\mathrm{n} / \mathrm{a}$ & $\mathrm{n} / \mathrm{a}$ & $\mathrm{n} / \mathrm{a}$ & $\mathrm{n} / \mathrm{a}$ & 9.7 & $<1.0^{d}$ & $e$ & $e$ \\
\hline$(3 R, 4 R)-22$ & endo & 21.2 & $\mathrm{n} / \mathrm{a}$ & 24.4 & $\mathrm{n} / \mathrm{a}$ & $\mathrm{n} / \mathrm{a}$ & $<1.0^{d}$ & $\mathrm{n} / \mathrm{a}$ & $<1.0^{d}$ \\
\hline
\end{tabular}

${ }^{a 1} \mathrm{H}-{ }^{1} \mathrm{H}$ couplings measured using PSYCHEDELIC ${ }^{70}{ }^{1} \mathrm{H}-{ }^{19} \mathrm{~F}$ couplings were read from the $1 \mathrm{D}{ }^{1} \mathrm{H}$ spectrum on the $\mathrm{H}_{\alpha}$ proton. ${ }^{b}$ Bias refers to the conformational preference of the nonfluorinated $\mathrm{N}$-acetyl proline methyl ester as a reference. "Cisoid" indicates that the coupled atoms are on the same side of the proline ring, whereas "transoid" indicates that the coupled atoms are on different sides of the ring. ${ }^{d}$ Value smaller than signal line width. ${ }^{e}$ Degenerate $\mathrm{H}_{\beta}$ chemical shifts. Individual couplings could not be extracted.

Finally, the calculated ratios between $\mathrm{C}^{\gamma}$ endo and $\mathrm{C}^{\gamma}$ exo puckers using DFT with chloroform or water as an implicit solvent are provided (Table 3). Unmodified proline has a higher preference for the $\mathrm{C}^{\gamma}$ endo than the $\mathrm{C}^{\gamma}$ exo pucker. ${ }^{9}$ Both $(4 S)$ - and (3S)-fluoroprolines, $\mathbf{5 4}$ and $\mathbf{5 5}$, strongly bias these pucker ratios to the $\mathrm{C}^{\gamma}$ endo form, with negligible $\mathrm{C}^{\gamma}$ exo pucker populations, both in chloroform and water. ${ }^{21}$ As expected, the $(3 R, 4 R)$-difluoroproline variant $\mathbf{2 2}$ is heavily biased to the $\mathrm{C}^{\gamma}$ endo pucker as well, with essentially the same $\mathrm{C}^{\gamma}$ endo/ $\mathrm{C}^{\gamma}$ exo ratio as that of its (3S)- and (4S)-progenitors. The (4R)- and (3R)-fluoroprolines, $\mathbf{5 2}$ and 53, are biased to the $C^{\gamma}$ exo pucker relative to Pro, albeit to different degrees. Where the $(4 R)$ variant $\mathbf{5 2}$ shows a similar $\mathrm{C}^{\gamma}$ exo bias in both solvents and for both trans and cis forms, the cis rotamer of the (3R)-variant 53 shows a high $\mathrm{C}^{\gamma}$ exo bias in chloroform, but a low bias in water. The $(3 S, 4 S)$-difluorinated proline 21 shows a bias to the $\mathrm{C}^{\gamma}$ exo pucker in the same order of magnitude as its progenitors. Interestingly, especially in the cis rotamer, the $\mathrm{C}^{\gamma}$ exo pucker is highly populated in both solvents, even higher than in its trans rotamer and than in its progenitors.

Experimental verification of these computational results can in principle occur via analysis of vicinal scalar couplings. Unfortunately, ${ }^{3} J_{\mathrm{FF}}$ couplings are known not to be practically exploitable to assess the dihedral angle, ${ }^{69}$ while quantitatively calculating the ring pucker from experimental ${ }^{3} J_{\mathrm{HF}}$ and ${ }^{3} J_{\mathrm{HH}}$ couplings was in our hands found not to be reliable due to the limited accuracy of Karplus relations for difluorinated fivemembered pyrrolidine rings. Instead, these couplings can 
qualitatively be compared to those of the monofluorinated progenitors (Table 4), bearing in mind that the different fluorine substitution patterns may significantly influence the Karplus relation. The (4R)- and (4S)-monofluoroprolines, which are established as strongly biased to, respectively, $\mathrm{C}^{\gamma}$ exo and $\mathrm{C}^{\gamma}$ endo, clearly display distinct transoid ${ }^{3} \mathrm{~J}_{\mathrm{H}_{\alpha} \mathrm{H}_{\beta}}$ coupling constants of $8.2_{\text {cis }} / 10.1_{\text {trans }} \mathrm{Hz}$ and $<1.0 \mathrm{~Hz}$, respectively, implying this coupling provides a sensitive measure for the endo/exo ratio. Both the similar small magnitude of this coupling in (3S)-monofluoroproline, known to have a pronounced $\mathrm{C}^{\gamma}$ endo pucker, ${ }^{21}$ and the larger values found for proline $\left(2.6_{\text {cis }} / 4.7_{\text {trans }} \mathrm{Hz}\right)$, consistent with intermediate endo/exo ratios and a higher endo population in the cis-form, confirm the relevance of ${ }^{3} J_{\mathrm{H}_{\alpha} \mathrm{H}_{\beta}}$ coupling constants for a qualitative analysis of a fluorinated proline ring pucker. Hence, given the $(3 R, 4 R)$-difluorinated variant 22 also shows a small ${ }^{3} J_{\mathrm{H}_{\alpha} \mathrm{H}_{\beta}}$ coupling value of $<0.5 \mathrm{~Hz}$, its calculated preference for a $\mathrm{C}^{\gamma}$ endo pucker is consistent with these experimental data.

In contrast, the cisoid ${ }^{3} \mathrm{~J}_{\mathrm{H}_{\alpha} \mathrm{H}_{\beta}}$ coupling constants of the (4R)and $(4 S)$-fluoroprolines and proline show similar values of 8.7 cis $/ 7.8_{\text {trans }} \mathrm{Hz}, 9.7 \mathrm{~Hz}$, and $8.9_{\text {cis }} / 8.8_{\text {trans }} \mathrm{Hz}$, respectively, implying this coupling is not very sensitive to the endo/exo ratio. Indeed, both the $(3 R)$-fluoroproline, known to prefer an exo pucker, $^{21}$ and the $(3 S, 4 S)$-difluoroproline show lower cisoid ${ }^{3} J_{\mathrm{H}_{\alpha} \mathrm{H}_{\beta}}$ couplings of $5.1 / 4.8 \mathrm{~Hz}$ and $5.2 / 5.0 \mathrm{~Hz}$, respectively, which suggests the fluorine substitution pattern is in this case the most significant factor determining the value. Nevertheless, the similarity of both the ${ }^{3} \mathrm{~J}_{\mathrm{H}_{\alpha} \mathrm{H}_{\beta}}$ and ${ }^{3} J_{\mathrm{H}_{\alpha} \mathrm{F}_{\beta}}$ couplings observed for the $(3 R)$ - and $(3 S, 4 S)$-variants suggests both fluoroprolines have mostly similar endo/exo ratios. In addition, these couplings differ significantly with those of the $(3 S, 4 R)$-variant, which is expected given the latter displays virtually no pucker preference.

The clear $\mathrm{C}^{\gamma}$ exo pucker bias observed for $(3 S, 4 S)$ difluorinated proline $\mathbf{2 1}$ in solution by NMR is also observed in its crystal structure (Figure 4). A single crystal of 22 was not obtained, but the $C^{\gamma}$ endo pucker bias of the $(3 R, 4 R)$ difluoroproline ring could be observed in the crystal structure of its N-Boc-protected precursor 24a (Figure 3). It should be noted that the packing of molecules in the solid state, and their resulting conformations, is determined from the sum of a multitude of inter- and intramolecular interactions, and often deviates from the conformation in solution, which in turn is typically solvent-dependent. With this caveat in mind, the observed conformations in the crystal structures strongly suggest that the 3,4-difluorination instills the expected conformational bias.

\section{DISCUSSION}

The potential of fluorinated prolines as tools for protein research has a long track record. Next to the well-known example of collagen, stabilized forms of proteins such as barstar, $^{13}$ ubiquitin, ${ }^{71}$ Trp cage mini protein, ${ }^{72}$ and $\mathrm{GFP}^{73}$ incorporating 4-fluoroprolines were obtained with the $\mathrm{C}_{4}$ stereochemistry selected to reinforce the pucker observed in the native protein. Both 3- and 4-monofluorinated prolines have been used to probe the effect of $\beta$-turn stability on the self-assembly of elastin peptide mimics. ${ }^{68}$ Accelerated peptide folding, as a consequence of the accelerated cis/trans kinetics, was observed when fluoroprolines were integrated in thioredoxin (Trx), ${ }^{74} \beta 2$-microglobulin $(\beta 2 \mathrm{~m}),{ }^{75}$ and ribonu- clease (RNase) A. ${ }^{76}$ Fluorinated prolines have also been used to reveal the relevance of a proline ring pucker in ribosomal peptide synthesis. ${ }^{77,78}$ The extended range of cis/trans isomerization kinetics offered by the 3,4-difluoroprolines, in conjunction with either a bias to trans and the $\mathrm{C}^{\gamma}$ exo pucker, to $c i s$ and the $\mathrm{C}^{\gamma}$ endo pucker, or a similar structural preference to proline, clearly will be of interest within such studies, allowing us to deconvolute the roles of ring pucker and cis/ trans preferences from isomerization kinetics.

Recently, Bernardes and Corzana and co-workers used a rational Pro-to-FPro substitution to stabilize an antigenantibody complex. ${ }^{79}$ As a result of its proximity to a highly electronegative fluorine, the polarization of a nearby $\mathrm{CH}$ bond was increased. This led to an enhanced $\mathrm{CH}-\pi$ interaction, which stabilized the antigen-antibody complex. A similar improved $\mathrm{CH}-\pi$ interaction has been observed between a fluoroproline-modified phosphopeptide and the WW domain of Pin $1 .{ }^{80}$ Clearly, 3,4-difluorinated proline analogues, especially with a 3,4-cis stereochemistry, will be of great interest in that regard, as enhanced $\mathrm{C}-\mathrm{H}$ polarization and thus enhanced $\mathrm{CH}-\pi$ interactions can be expected. ${ }^{81}$

Regarding the use of fluoroprolines as ${ }^{19} \mathrm{~F}$ NMR reporters, the simultaneous fluorination at the 3- and 4-positions provides for very distinct chemical shifts compared to the monofluorinated progenitors. The experimental ${ }^{19} \mathrm{~F}$ chemical shifts and ${ }^{n} J_{\mathrm{FF}}$ coupling constants for the $\mathrm{N}$-Ac-X-OMe model compounds the $(4,4)$-difluoroproline, $(3 S, 4 R),(3 R, 4 R)-$, and $(3 S, 4 S)-3,4$-difluorinated prolines, and their monofluorinated progenitors are shown in Table 5. For all 3,4-difluoroprolines,

Table 5. Fluorine Chemical Shift Values of Fluorinated $\mathrm{N}$ Ac-X-OMe Derivatives $\left(\mathrm{D}_{2} \mathrm{O}\right)$

\begin{tabular}{lllllr} 
& \multicolumn{2}{c}{${ }^{19} \mathrm{~F} \delta / \mathrm{ppm}(\mathrm{F} 3, \mathrm{~F} 4)$} & & \multicolumn{2}{c}{${ }^{n} J_{\mathrm{FF}}(\mathrm{Hz})$} \\
\cline { 2 - 3 } \cline { 5 - 6 } compound & \multicolumn{1}{c}{$c i s$ amide } & trans amide & & $\begin{array}{c}c i s \\
\text { amide }\end{array}$ & $\begin{array}{c}\text { trans } \\
\text { amide }\end{array}$ \\
$(3 R)-53$ & -184.4 & -186.4 & & & \\
$(3 S)-55$ & -176.8 & -175.7 & & & \\
$(4 R)-52$ & -177.9 & -177.0 & & & \\
$(4 S)-54$ & -173.1 & -172.9 & & \\
$(3 S, 4 S)-21$ & $-195.0,-194.3$ & $-197.2,-193.3$ & & 11.1 & 11.4 \\
$(3 R, 4 R)-22$ & $-190.6,-187.7$ & $-189.1,-188.4$ & & 12.9 & 13.0 \\
$(3 S, 4 R)-56$ & $-208.5,-200.3$ & $-210.4,-203.3$ & & 5.8 & 4.7 \\
$(4,4)-57$ & $-101.4,^{a}-96.7^{b}$ & $-98.2^{a}-99.1^{b}$ & & 236.0 & 233.7 \\
${ }^{a}$ pro- $R$ F4. ${ }^{b}{ }^{b}$ pro-S F4. & & & & \\
\hline
\end{tabular}

the homonuclear coupling constant between the vicinal ${ }^{19} \mathrm{~F}$ nuclei is small, as opposed to that of the geminal difluorinated $(4,4)$-variant. This property is very useful for advanced ${ }^{19} \mathrm{~F}$ NMR experiments, as it minimizes any potential complications from $J$ modulation during spin-echo pulse sequences, or from second-order effects, which is an issue in geminal difluorinated prolines. ${ }^{30}$ In addition, the 3,4-difluorinated derivatives have very distinct ${ }^{19} \mathrm{~F}$ chemical shift values compared to their monofluorinated progenitors, even though they possess similar structural properties. The 3,4-difluoroprolines can thus be used complementary to the monofluoroprolines for ${ }^{19} \mathrm{~F}$ NMR purposes, allowing for the design of combinatorial incorporation schemes aimed at studying poly proline- and prolinerich sequences, due to maximum chemical shift dispersion between these residues, but with minimal complications from homonuclear couplings. 


\section{Scheme 10. Summary Scheme for the Synthesis of Protected 3,4-Difluoroprolines}
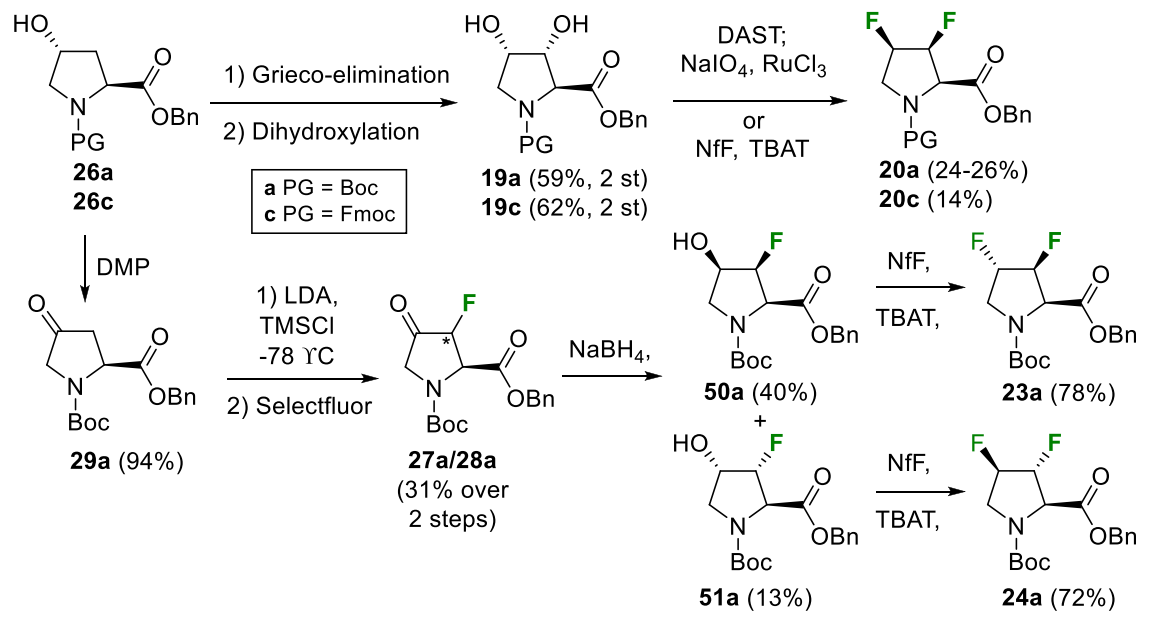

\section{CONCLUSION}

As part of a program to expand the scope of available fluorinated prolines, we report here in full the effective syntheses of three 3,4-difluorinated proline analogues (as summarized in Scheme 10). In addition, we report the first conformational characterization (trans/cis ratios and isomerization kinetics, and ring pucker preferences) of the $(3 R, 4 R)$ and $(3 S, 4 S)$-3,4-difluoroproline analogues.

The $(3 S, 4 R)$-difluorinated proline derivative could not be synthesized directly from 3,4-dehydroproline, or from the 3,4epoxyproline derivative, with the former being unreactive under conditions of alkene difluorination or halofluorination and the latter typically suffering from aromatization, leading to pyrrole derivatives. However, a direct bis-deoxyfluorination strategy with the easily accessible 3,4-dihydroxyproline as a substrate led to the desired target using both NfF and DAST, with the former giving the highest yield when $N$-Boc was used as a protecting group and the latter suitable with an $\mathrm{N}$-Fmocprotecting group. Yields were low $(26 \%$ and $14 \%$, respectively), but as only two transformations were required from the protected 3,4-dehydroproline, gram quantities are easily available. In this context, we report that the direct synthesis of Fmoc-protected 3,4-dehydroproline from the corresponding 4-hydroxyproline is possible using the one-pot Grieco elimination procedure, in contrast to the usually employed basic conditions.

The $(3 R, 4 R)$ - and the novel $(3 S, 4 S)$-difluorinated proline derivatives were synthesized using a two-step fluorination strategy: the first being an electrophilic fluorination starting from protected 4-ketoproline and the second by a DASTmediated deoxyfluorination after 4-ketoreduction. Hence, starting from 26a, the $(3 S, 4 S)$ - and $(3 R, 4 R)-N$-Boc 3,4difluoroproline benzyl esters were obtained in an overall yield of $9 \%$ for $\mathbf{2 3 a}$ and $3 \%$ for $\mathbf{2 4 a}$, in a combined 5 steps (with three common steps before diastereomer separation). It may be pointed out that the linear 10-step Fleet synthesis ${ }^{37}$ of $(3 R, 4 R)-N-B n$ difluoroproline methyl ester 17 (cf. Scheme 1) has a higher overall yield (24\%), although this delivers a single diastereomer only. In addition, Ciulli reported higher yields for the electrophilic fluorination step. ${ }^{39}$ X-ray crystallographic analysis allowed unambiguous determination of the relative configuration of the obtained 3,4-difluoroprolines.

Due to the opposing conformational effects of each individual fluorine in the $(3 S, 4 R)$-difluorinated proline derivative, this analogue has previously been described as having a minimal conformational bias to proline. ${ }^{34}$ In contrast, it is shown here that a combination of 3- and 4-fluorine substitutions with similar preorganizing effects results in 3,4difluorinated proline derivatives with similar conformational preferences as monofluorinated prolines. While the $(3 R, 4 R)$ difluorinated proline derivative resembles most closely the (4S)-fluoroproline, the $(3 S, 4 S)$-difluorinated proline derivative resembles the $(4 R)$-fluoroproline, though with a somewhat higher preference for a $\mathrm{C}^{\gamma}$ exo pucker in its cis rotamer. Given the distinct ${ }^{19} \mathrm{~F}$ chemical shifts of both 3,4-difluorinated derivatives to their monofluorinated progenitors, they will be of interest for multiresidue fluorine-labeling strategies, for instance, in the study of repetitive or low-complexity protein sequences, where similar conformational preorganizing effects are desired, but distinct residue-specific ${ }^{19} \mathrm{~F}$ NMR chemical shifts are needed.

A clearer difference between the 3,4-difluoprolines and their monofluorinated progenitors is the faster amide rotamer isomerization rates. This is expected given the larger electron-withdrawing effect of two fluorines compared to that of one. Especially the $(3 S, 4 S)$ variant shows a remarkably high isomerization rate, higher than any previously described difluorinated variant. These new variants will thus be very useful toward studying the role of Xaa-Pro cis/trans isomerization kinetics for biological function, ${ }^{82}$ protein folding, ${ }^{83}$ or amyloid assembly. ${ }^{75}$

Applications of the 3,4-difluoroprolines are in progress and will be reported in due course, as are deeper investigations on revealing the structural origins of their conformational properties and cis/trans isomerization kinetics.

\section{EXPERIMENTAL SECTION}

General Conditions. All air/moisture-sensitive reactions were carried out under an inert atmosphere (Ar), in dried glassware. Dry $\mathrm{CH}_{2} \mathrm{Cl}_{2}$, THF, $\mathrm{MeOH}$, and hexane were bought from commercial suppliers and used as received. TLC was performed on aluminumprecoated plates coated with silica gel 60 with an F254 indicator; visualized under UV light $(254 \mathrm{~nm})$ and/or by staining with $\mathrm{KMnO}_{4}$ ( $10 \% \mathrm{aq})$. Flash column chromatography was performed with SigmaAldrich 60 silica gel $(40-63 \mu \mathrm{m})$. Preparative HPLC was carried out using a Biorad Bio-Sil D 90-10 column $(250 \mathrm{~mm} \times 22 \mathrm{~mm}$ at $15 \mathrm{~mL}$ $\left.\min ^{-1}\right)$. High-resolution MS samples were analyzed using a MaXis (Bruker Daltonics, Bremen, Germany) mass spectrometer equipped with a time of flight (TOF) analyzer. Samples were introduced to the 
mass spectrometer via a Dionex Ultimate 3000 autosampler and uHPLC pump and eluted in $5 \mathrm{~min}$ at $0.6 \mathrm{~mL}$ min using a gradient of $20 \%$ acetonitrile $(0.2 \%$ formic acid $)$ to $100 \%$ acetonitrile $(0.2 \%$ formic acid) through an Acquity UPLC BEH C18 (Waters) $1.7 \mu \mathrm{m} 50 \mathrm{~mm} \times$ $2.1 \mathrm{~mm}$ column. High-resolution mass spectra were recorded using positive ion electrospray ionization. ${ }^{1} \mathrm{H},{ }^{19} \mathrm{~F}$, and ${ }^{13} \mathrm{C}$ NMR spectra were recorded at room temperature on a Bruker Ultrashield 400 or $500 \mathrm{MHz}$ spectrometer. ${ }^{1} \mathrm{H}$ and ${ }^{13} \mathrm{C}$ chemical shifts $(\delta)$ are quoted in ppm relative to residual solvent peaks as appropriate. ${ }^{19} \mathrm{~F}$ spectra were externally referenced to $\mathrm{CFCl}_{3}$. The coupling constants $(J)$ are given in hertz $(\mathrm{Hz})$. The NMR signals were designated as follows: $s$ (singlet), $\mathrm{d}$ (doublet), $\mathrm{t}$ (triplet), $\mathrm{q}$ (quartet), quin (quintet), sxt (sextet), spt (septet), m (multiplet), or a combination of the above. For all compounds, a detailed peak assignment was performed through the combined use of HSQC, HMBC, NOESY, and COSY NMR experiments.

$N$-(Acetyl)-(2S,4R)-4-(methanesulfonyloxy)proline Benzyl Ester (30b) (Scheme 3). Alcohol 26b (14.5 g, $55.1 \mathrm{mmol}$ ) was dissolved in pyridine $(150 \mathrm{~mL})$ and cooled to $0{ }^{\circ} \mathrm{C}$. Mesyl chloride $(6.82 \mathrm{~mL}, 88.1 \mathrm{mmol})$ was added dropwise, and the mixture was allowed to warm to room temperature. TLC analysis indicated the reaction was finished after $7 \mathrm{~h}$. Subsequently, the mixture was cooled to $0{ }^{\circ} \mathrm{C}$ and quenched with a solution of $10 \% \mathrm{H}_{2} \mathrm{O}$ in pyridine (50 $\mathrm{mL}$ ). The solvent was evaporated in vacuo, the crude product redissolved in $\mathrm{H}_{2} \mathrm{O}(80 \mathrm{~mL})$ and the aqueous layer extracted with DCM $(3 \times 80 \mathrm{~mL})$. The combined organic layers were washed with a saturated aqueous solution of $\mathrm{NaHCO}_{3}(60 \mathrm{~mL})$ and brine $(60 \mathrm{~mL})$, dried over $\mathrm{MgSO}_{4}$, and evaporated in vacuo to yield $30 \mathrm{~b}$ (18.8 g, quant) as an off-white solid: ${ }^{1} \mathrm{H}$ NMR $\left(400 \mathrm{MHz}, \mathrm{CDCl}_{3}\right)(78: 22$ rotamer ratio) $\delta 7.42-7.31(\mathrm{~m}, 5 \mathrm{H}$ major $\mathrm{Ph}+5 \mathrm{H}$ minor $\mathrm{Ph}), 5.36-$ $5.30\left(\mathrm{~m}, 1 \mathrm{H}\right.$, major $\left.\mathrm{C}_{\gamma} \underline{\mathrm{H}}\right), 5.29-5.24\left(\mathrm{~m}, 1 \mathrm{H}\right.$, minor $\left.\mathrm{C}_{\gamma} \underline{\mathrm{H}}\right), 5.23(\mathrm{~d}, J$ $=12.4 \mathrm{~Hz}, 1 \mathrm{H}$, major $\left.\mathrm{CHH}^{\prime} \mathrm{Ph}\right), 5.23\left(\mathrm{~s}, 2 \mathrm{H}\right.$, minor $\left.\mathrm{CH}_{2} \mathrm{Ph}\right), 5.19(\mathrm{~d}$, $J=12.4 \mathrm{~Hz}, 1 \mathrm{H}$, major $\left.\mathrm{CH}^{\prime} \mathrm{Ph}\right), 4.61(\mathrm{t}, J=8.0 \mathrm{~Hz}, 1 \mathrm{H}$, major $\left.\mathrm{C}_{\alpha} \underline{\mathrm{H}}\right), 4.58\left(\mathrm{dd}, J=8.1,7.2 \mathrm{~Hz}, 1 \mathrm{H}\right.$, minor $\left.\mathrm{C}_{\alpha} \underline{\mathrm{H}}\right), 4.15(\mathrm{dt}, J=13.5$, $2.1 \mathrm{~Hz}, 1 \mathrm{H}$, minor $\left.\mathrm{C}_{\delta} \mathrm{HH}^{\prime}\right), 3.94(\mathrm{dd}, J=12.2,4.5 \mathrm{~Hz}, 1 \mathrm{H}$, major $\left.\mathrm{C}_{\delta} \mathrm{H}^{\prime}\right), 3.87\left(\mathrm{dt}, J=12.0,2.1 \mathrm{~Hz}, 1 \mathrm{H}\right.$, major $\left.\mathrm{C}_{\delta} \underline{\mathrm{H}} \mathrm{H}^{\prime}\right), 3.68(\mathrm{dd}, J=$ $13.5,4.6 \mathrm{~Hz}, 1 \mathrm{H}$, minor $\left.\mathrm{C}_{\delta} \mathrm{H}^{\prime}\right), 3.06\left(\mathrm{~s}, 3 \mathrm{H}\right.$, major $\left.\underline{\mathrm{H}}_{3} \mathrm{C}-\mathrm{SO}_{2}\right), 3.04$ (s, $3 \mathrm{H}$, minor $\underline{\mathrm{H}}_{3} \mathrm{C}-\mathrm{SO}_{2}$ ), 2.80 (dddd, $J=14.4,8.3,3.1,2.1 \mathrm{~Hz}, 1 \mathrm{H}$, minor $\mathrm{C}_{\beta} \underline{\mathrm{HH}^{\prime}}$ ), 2.59 (dddd, $J=14.3,8.2,3.2,1.6 \mathrm{~Hz}, 1 \mathrm{H}$, major $\mathrm{C}_{\beta} \underline{\mathrm{HH}^{\prime}}$ ), $2.42\left(\mathrm{ddd}, J=14.3,6.9,5.3 \mathrm{~Hz}, 1 \mathrm{H}\right.$, minor $\mathrm{C}_{\beta} \mathrm{H}^{\prime}$ ), 2.25 (ddd, $J=14.2,7.8,5.1 \mathrm{~Hz}, 1 \mathrm{H}$, major $\mathrm{C}_{\beta} \mathrm{HH}^{\prime}$ ), 2.11 (s, $3 \mathrm{H}$, major $\left.\mathrm{CO}-\mathrm{CH}_{3}\right), 1.93\left(\mathrm{~s}, 3 \mathrm{H}\right.$, minor $\left.\mathrm{CO}-\mathrm{CH}_{3}\right) \mathrm{ppm} ;{ }^{13} \mathrm{C}\left\{{ }^{1} \mathrm{H}\right\}$ NMR $(100$ $\mathrm{MHz}, \mathrm{CDCl}_{3}$ ), ${ }^{13} \mathrm{C}$ NMR $\delta 171.3$ (major $\mathrm{C}_{\alpha}-\mathrm{CO}_{2}$ ), 171.1 (minor $\mathrm{C}_{\alpha^{-}}$ $\left.\mathrm{CO}_{2}\right), 169.8($ minor $\mathrm{N}-\underline{\mathrm{COCH}} 3), 169.3\left(\right.$ major $\left.\mathrm{N}-\underline{\mathrm{COCH}}{ }_{3}\right), 135.4$ (major $\left.\underline{\mathrm{C}}_{\mathrm{q}, \mathrm{Ph}}\right), 134.7\left(\right.$ minor $\left.\underline{\mathrm{C}}_{\mathrm{q}, \mathrm{Ph}}\right), 128.9+128.8+128.6+128.5+$ $128.4+128.2$ (major and minor overlap, $\underline{\mathrm{C}}_{\mathrm{Ph}}$ ), 77.1 (major $\underline{\mathrm{C}}_{\gamma} \mathrm{H}$ ), 77.2 (minor $\left.\underline{\mathrm{C}}_{\gamma} \mathrm{H}\right), 67.8$ (minor $\left.\underline{\mathrm{CH}}_{2} \mathrm{Ph}\right), 67.2$ (major $\left.\underline{\mathrm{CH}}_{2} \mathrm{Ph}\right), 58.2$ (minor $\underline{\mathrm{C}}_{\alpha} \mathrm{H}$ ), 57.1 (major $\underline{\mathrm{C}}_{\alpha} \mathrm{H}$ ), 53.6 (major $\underline{\mathrm{C}}_{\delta} \mathrm{H}_{2}$ ), 51.8 (minor $\underline{\mathrm{C}}_{\delta} \mathrm{H}_{2}$ ), 38.8 (minor $\mathrm{H}_{3} \underline{\mathrm{C}}-\mathrm{SO}_{2}$ ), 38.7 (major $\mathrm{H}_{3} \underline{\mathrm{C}}-\mathrm{SO}_{2}$ ), 38.1 (minor $\underline{\mathrm{C}}_{\beta} \mathrm{H}_{2}$ ), 35.7 (major $\underline{\mathrm{C}}_{\beta} \mathrm{H}_{2}$ ), 22.2 (major $\mathrm{COC}_{3}$ ), 21.6 (minor $\left.\mathrm{COCH}_{3}\right)$ ppm; $[\alpha]_{\mathrm{D}}^{21}-43\left(\mathrm{c} 0.8, \mathrm{CHCl}_{3}\right) ; \mathrm{mp} 96-100{ }^{\circ} \mathrm{C} ; R_{f} 0.43$ (hexane/acetone 50:50); MS (ESI) $(\mathrm{m} / z) 342.3[\mathrm{M}+\mathrm{H}]^{+}, 364.3[\mathrm{M}$ $+\mathrm{Na}]^{+}$; HRMS (ESI) for $\mathrm{C}_{15} \mathrm{H}_{20} \mathrm{NO}_{6} \mathrm{~S}[\mathrm{M}+\mathrm{H}]^{+}$calcd for 342.1006, found 342.1007; IR 1743 (s), 1651 (s), 1354 (s), $1172(\mathrm{~s}) \mathrm{cm}^{-1}$.

$(+)-N$-(Acetyl)-3,4-dehydroproline Benzyl Ester (25b) and ( \pm )- $N$-(Acetyl)-4,5-dehydroproline Benzyl Ester (31b) (Scheme 3). Mesylate $30 \mathrm{~b}(18.8 \mathrm{~g}, 55.3 \mathrm{mmol})$ was dissolved in toluene (200 $\mathrm{mL}), \mathrm{DBU}(24.8 \mathrm{~mL}, 165 \mathrm{mmol})$ was added, and the mixture was refluxed at $110^{\circ} \mathrm{C}$. After $14 \mathrm{~h}$, the solvent was evaporated in vacuo. Next, the crude product was redissolved in DCM $(250 \mathrm{~mL})$ and washed with a saturated aqueous solution of $\mathrm{NaHCO}_{3}(2 \times 150 \mathrm{~mL})$ and brine $(150 \mathrm{~mL})$. The organic layer was dried over $\mathrm{MgSO}_{4}$ and evaporated in vacuo. Purification by flash chromatography (hexane/ acetone 65:35) and Biotage (hexane/acetone gradient) yielded alkene ( \pm -25b (7.19 g, 53\%) and alkene $\mathbf{3 1 b}(3.33 \mathrm{~g}, 25 \%)$ as colorless oils. Data for ( \pm )-N-(Acetyl)-3,4-dehydroproline Benzyl Ester (25b): ${ }^{1} \mathrm{H} \mathrm{NMR}\left(400 \mathrm{MHz}, \mathrm{CDCl}_{3}\right)$ (76:24 rotamer ratio) $\delta 7.41-7.29(\mathrm{~m}$, $5 \mathrm{H}$ major $\mathrm{Ph}+5 \mathrm{H}$ minor $\mathrm{Ph}$ ), 6.05 (app. dq, $J=6.3,2.1 \mathrm{~Hz}, 1 \mathrm{H}$, minor $\mathrm{C}_{\beta} \underline{\mathrm{H}}$ ), 5.99 (app. dq, $J=6.3,2.1 \mathrm{~Hz}, 1 \mathrm{H}$, major $\mathrm{C}_{\beta} \underline{\mathrm{H}}$ ), 5.85$5.78\left(\mathrm{~m}, 1 \mathrm{H}\right.$ major $\mathrm{C}_{\gamma} \underline{\mathrm{H}}$ and $1 \mathrm{H}$ minor $\left.\mathrm{C}_{\gamma} \underline{\mathrm{H}}\right), 5.25-5.10(\mathrm{~m}, 2 \mathrm{H}$ major $\mathrm{CH}_{2} \mathrm{Ph}+2 \mathrm{H}$ minor $\mathrm{C}_{2} \mathrm{Ph}+1 \mathrm{H}$ major $\mathrm{C}_{\alpha} \underline{\mathrm{H}}+1 \mathrm{H}$ minor $\left.\mathrm{C}_{\alpha} \underline{\mathrm{H}}\right), 4.46-4.23\left(\mathrm{~m}, 2 \mathrm{H}\right.$ major $\mathrm{C}_{\delta} \underline{\mathrm{H}}_{2}$ and $2 \mathrm{H}$ minor $\left.\mathrm{C}_{\delta} \underline{\mathrm{H}}_{2}\right), 2.12(\mathrm{~s}$, $3 \mathrm{H}$, major $\left.\mathrm{CH}_{3}\right), 1.93$ (s, 3H, minor $\left.\mathrm{C}_{3}\right)$ ppm; ${ }^{13} \mathrm{C}\left\{{ }^{1} \mathrm{H}\right\}$ NMR (100 $\mathrm{MHz}, \mathrm{CDCl}_{3}$ ), ${ }^{13} \mathrm{C}$ NMR (76:24 rotamer ratio) $\delta 169.6$ (minor $\mathrm{C}_{\alpha^{-}}$ $\mathrm{CO}_{2}$ ), 169.5 (major $\mathrm{C}_{\alpha}-\mathrm{CO}_{2}$ ), 169.3 (minor $\mathrm{N}-\mathrm{COCH}_{3}$ ), 169.0 (major $\left.\mathrm{N}-\underline{\mathrm{COCH}}{ }_{3}\right), 135.6$ (major $\left.\underline{\mathrm{C}}_{\mathrm{q}, \mathrm{Ph}}\right), 135.1\left(\right.$ minor $\left.\underline{\mathrm{C}}_{\mathrm{q}, \mathrm{Ph}}\right), 129.8$ (minor $\underline{\mathrm{C}}_{\beta} \mathrm{H}$ ), $128.6\left(\right.$ major $\left.\underline{\mathrm{C}}_{\beta} \mathrm{H}\right), 128.72+128.68+128.3+128.2+$ 128.0 (major and minor overlap, $\mathrm{CH}_{\mathrm{Ph}}$ ), 125.3 (major $\underline{\mathrm{C}}_{\gamma} \mathrm{H}$ ), 124.3 (minor $\underline{\mathrm{C}}_{\gamma} \mathrm{H}$ ), 67.5 (minor $\underline{\mathrm{CH}}_{2} \mathrm{Ph}$ ), 67.2 (minor $\underline{\mathrm{C}}_{\alpha} \mathrm{H}$ ), 67.0 (major $\left.\underline{\mathrm{CH}}_{2} \mathrm{Ph}\right), 66.2\left(\right.$ major $\left.\underline{\mathrm{C}}_{\alpha} \mathrm{H}\right), 54.3$ (major $\left.\underline{\mathrm{C}}_{\delta} \mathrm{H}_{2}\right), 53.5$ (minor $\left.\underline{\mathrm{C}}_{\delta} \mathrm{H}_{2}\right)$, 21.8 (major $\left.\underline{\mathrm{CH}}_{3}\right), 21.7$ (minor $\left.\mathrm{CH}_{3}\right)$ ppm; $R_{f} 0.64$ (hexane/acetone 50:50); MS (ESI) $(m / z) 246.1[\mathrm{M}+\mathrm{H}]^{+}, 268.1[\mathrm{M}+\mathrm{Na}]^{+}$; HRMS (ESI) for $\mathrm{C}_{14} \mathrm{H}_{16} \mathrm{NO}_{3}[\mathrm{M}+\mathrm{H}]^{+}$calcd for 246.1125, found 246.1126; IR 1747 (s), $1654(\mathrm{~s}), 1619(\mathrm{~m}) \mathrm{cm}^{-1}$.

Data for Partially Racemized N-(Acetyl)-4,5-dehydroproline Benzyl Ester (31b): ${ }^{1} \mathrm{H}$ NMR (400 $\mathrm{MHz}, \mathrm{CDCl}_{3}$ ) (89:11 rotamer ratio) $\delta 7.42-7.30(\mathrm{~m}, 5 \mathrm{H}$ major $\mathrm{Ph}+5 \mathrm{H}$ minor $\mathrm{Ph}), 7.02$ (app. dt, $J$ $=4.3,2.2 \mathrm{~Hz}, 1 \mathrm{H}$, minor $\left.\mathrm{C}_{\delta} \underline{\mathrm{H}}\right), 6.51(\mathrm{br} \mathrm{dt}, J=4.3,2.2 \mathrm{~Hz}, 1 \mathrm{H}$, major $\left.\mathrm{C}_{\delta} \underline{\mathrm{H}}\right), 5.29-5.15\left(\mathrm{~m}, 2 \mathrm{H}\right.$ major $\mathrm{CH}_{2} \mathrm{Ph}$ and $2 \mathrm{H}$ minor $\mathrm{CH}_{2} \mathrm{Ph}$ ), 5.15-5.12 (m, $1 \mathrm{H}$ major $\mathrm{C}_{\gamma} \underline{\mathrm{H}}$ and $1 \mathrm{H}$ minor $\mathrm{C}_{\gamma} \underline{\mathrm{H}}$ ), 4.89 (dd, $J=11.7$, $5.0 \mathrm{~Hz}, 1 \mathrm{H}$, major $\left.\mathrm{C}_{\alpha} \mathrm{H}\right), 4.70\left(\mathrm{dd}, J=11.3,3.6 \mathrm{~Hz}, 1 \mathrm{H}\right.$, minor $\left.\mathrm{C}_{\alpha} \underline{\mathrm{H}}\right)$, $3.21\left(\mathrm{~m}, 1 \mathrm{H}\right.$, minor $\left.\mathrm{C}_{\beta} \underline{\mathrm{HH}^{\prime}}\right), 3.03\left(\mathrm{~m}, 1 \mathrm{H}\right.$, major $\left.\mathrm{C}_{\beta} \underline{\mathrm{HH}^{\prime}}\right), 2.82(\mathrm{~m}$, $1 \mathrm{H}$, minor $\left.\mathrm{C}_{\beta} \mathrm{H}_{\mathrm{H}^{\prime}}\right), 2.62\left(\mathrm{~m}, 1 \mathrm{H}\right.$, major $\left.\mathrm{C}_{\beta} \mathrm{H}^{\prime}{ }^{\prime}\right), 2.17$ (s, 3H, major $\left.\mathrm{C}_{3}\right), 1.93$ (s, 3H, minor $\left.\mathrm{CH}_{3}\right)$ ppm; ${ }^{13} \mathrm{C}\left\{{ }^{1} \mathrm{H}\right\}$ NMR (100 MHz, $\mathrm{CDCl}_{3}$ ) (89:11 rotamer ratio) $\delta 171.1$ (minor $\mathrm{C}_{\alpha}-\underline{\mathrm{CO}_{2}}$ ), 170.8 (major

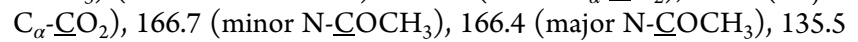
(major $\underline{\mathrm{C}}_{\mathrm{q}, \mathrm{Ph}}$ ), 135.3 (minor $\underline{\mathrm{C}}_{\mathrm{q}, \mathrm{Ph}}$ ), 129.7 (minor $\underline{\mathrm{C}}_{\delta} \mathrm{H}$ ), 129.5 (major $\underline{\mathrm{C}}_{\delta} \mathrm{H}$ ), $128.51+128.49+128.4+128.3+128.2+128.0$ (major and minor overlap, $\underline{\mathrm{CH}}_{\mathrm{Ph}}$ ), 108.8 (major $\left.\underline{\mathrm{C}}_{\gamma} \mathrm{H}\right), 107.7\left(\right.$ minor $\left.\underline{\mathrm{C}}_{\gamma} \mathrm{H}\right), 67.2$ (minor $\mathrm{CH}_{2} \mathrm{Ph}$ ), 66.9 (major $\mathrm{CH}_{2} \mathrm{Ph}$ ), 59.1 (minor $\underline{\mathrm{C}}_{\alpha} \mathrm{H}$ ), 57.7 (major $\underline{\mathrm{C}}_{\alpha} \mathrm{H}$ ), 36.1 (minor $\underline{\mathrm{C}}_{\beta} \mathrm{H}$ ), 33.7 (major $\underline{\mathrm{C}}_{\beta} \mathrm{H}$ ), 21.8 (minor $\mathrm{CH}_{3}$ ), 21.5 (major $\mathrm{CH}_{3}$ ) ppm; $R_{f} 0.72$ (hexane/acetone 50:50); MS (ESI) $(m / z) 246.1[\mathrm{M}+\mathrm{H}]^{+}, 268.1[\mathrm{M}+\mathrm{Na}]^{+}$; HRMS (ESI) for $\mathrm{C}_{14} \mathrm{H}_{16} \mathrm{NO}_{3}[\mathrm{M}+\mathrm{H}]^{+}$calcd for 246.1125, found 246.1127; IR 1739 (s), $1654(\mathrm{~s}), 1620(\mathrm{~m}) \mathrm{cm}^{-1}$.

$\mathrm{N}$-(9-Fluorenylmethyloxycarbonyl)-(2S)-3,4-dehydroproline Benzyl Ester (25c) (Scheme 3). At $0{ }^{\circ} \mathrm{C}$, tributylphosphine (0.98 $\mathrm{mL}, 3.93 \mathrm{mmol}$ ) and 2-nitrophenyl selenocyanate $(725.7 \mathrm{mg}, 3.20$ $\mathrm{mmol})$ were added to a solution of alcohol $26 \mathrm{c}(1.09 \mathrm{~g}, 2.46 \mathrm{mmol})$ in THF $(10.0 \mathrm{~mL})$. After the mixture was stirred at room temperature for $7 \mathrm{~h}$, TLC analysis indicated complete consumption of the starting material. Next, $\mathrm{H}_{2} \mathrm{O}_{2}(30 \% \mathrm{w} / \mathrm{w}, 10.0 \mathrm{~mL})$ was added, and the mixture was stirred overnight at room temperature. The reaction mixture was cooled on ice and slowly quenched with a saturated aqueous solution of $\mathrm{Na}_{2} \mathrm{~S}_{2} \mathrm{O}_{3}(20 \mathrm{~mL})$. The aqueous phase was extracted with DCM $(3 \times 25 \mathrm{~mL})$. The combined organic phases were washed with brine $(25 \mathrm{~mL})$ and dried over $\mathrm{MgSO}_{4}$, and the solvent was evaporated in vacuo. Purification by flash chromatography (hexane/EtOAc 90:10 to 75:25) yielded alkene 25c (815.5 mg, 78\%) as a light orange oil: ${ }^{1} \mathrm{H}$ NMR $\left(400 \mathrm{MHz}, \mathrm{CDCl}_{3}\right)(51: 49$ rotamer ratio) $\delta$ 7.83-7.23 (m, 13H major $\mathrm{Ar}-\mathrm{H}+13 \mathrm{H}$ minor $\mathrm{Ar}-\mathrm{H}), 6.06-$ $5.99\left(\mathrm{~m}, 1 \mathrm{H}\right.$ major $\mathrm{C}_{\gamma} \mathrm{H}+1 \mathrm{H}$ minor $\left.\mathrm{C}_{\gamma} \mathrm{H}\right), 5.85-5.76(\mathrm{~m}, 1 \mathrm{H}$ major $\mathrm{C}_{\beta} \mathrm{H}+1 \mathrm{H}$ minor $\left.\mathrm{C}_{\beta} \mathrm{H}\right), 5.26\left(\mathrm{~d}, J=12.2 \mathrm{~Hz}, 1 \mathrm{H}\right.$, major $\left.\mathrm{CH}^{\prime} \mathrm{Ph}\right)$, $5.18\left(\mathrm{~d}, J=12.5 \mathrm{~Hz}, 1 \mathrm{H}\right.$, major $\left.\mathrm{CH}_{\mathrm{H}^{\prime}} \mathrm{Ph}\right), 5.17(\mathrm{~d}, J=12.4 \mathrm{~Hz}, 1 \mathrm{H}$, minor $\left.\mathrm{CH}^{\prime} \mathrm{Ph}\right), 5.07\left(\mathrm{~d}, J=12.2 \mathrm{~Hz}, 1 \mathrm{H}\right.$, minor $\left.\mathrm{CH} \underline{H}^{\prime} \mathrm{Ph}\right), 5.23-$ $5.10\left(\mathrm{~m}, 1 \mathrm{H}\right.$ major $\mathrm{C}_{\alpha} \mathrm{H}+1 \mathrm{H}$ minor $\left.\mathrm{C}_{\alpha} \mathrm{H}\right), 4.55-4.26(\mathrm{~m}, 2 \mathrm{H}$ major $\mathrm{NCO}_{2}-\mathrm{CH}_{2}-\mathrm{CH}+2 \mathrm{H}$ minor $\mathrm{NCO}_{2}-\mathrm{CH}_{2}-\mathrm{CH}+1 \mathrm{H}$ major $\mathrm{NCO}_{2}-$ $\mathrm{CH}_{2}-\mathrm{CH}+2 \mathrm{H}$ major $\mathrm{C}_{\delta} \mathrm{H}_{2}+2 \mathrm{H}$ minor $\left.\mathrm{C}_{\delta} \mathrm{H}_{2}\right), 4.05(\mathrm{t}, J=6.9 \mathrm{~Hz}$, $1 \mathrm{H}$ minor $\left.\mathrm{NCO}_{2}-\mathrm{CH}_{2}-\mathrm{C} \underline{\mathrm{H}}\right) \mathrm{ppm} ;{ }^{13} \mathrm{C}\left\{{ }^{1} \mathrm{H}\right\}$ NMR $(100 \mathrm{MHz}$, $\mathrm{CDCl}_{3}$ ) (51:49 rotamer ratio) $\delta 169.8$ (major $\mathrm{C}_{\alpha}-\mathrm{CO}_{2}$ ), 169.7 (minor $\mathrm{C}_{\alpha}-\underline{\mathrm{CO}_{2}}$ ), $154.3\left(\right.$ major $\left.\mathrm{NCO}_{2}\right), 154.0\left(\right.$ minor $\left.\mathrm{NCO}_{2}\right), 144.13+$ $144.06+143.8+143.6$ (major and minor overlap, $\mathrm{C}_{\mathrm{q}, \mathrm{Fmoc}}$ ), $141.29+$ $141.27+141.26+141.19$ (major and minor overlap, $\mathrm{C}_{\mathrm{q}, \mathrm{Fmoc}}$ ), 135.5 (minor $\underline{\mathrm{C}}_{\mathrm{q}, \mathrm{Ph}}$ ), 135.3 (major $\underline{\mathrm{C}}_{\mathrm{q}, \mathrm{Ph}}$ ), 129.3 (minor $\mathrm{C}_{\gamma}$ ), 129.2 (major $\left.\mathrm{C}_{\gamma}\right), 128.5+128.34+128.25+128.1+128.0+127.69+127.65+$ $127.59+127.05+127.03+127.01+126.98+126.93+125.14+$ $125.08+125.0+124.9$ (major and minor overlap, $\underline{\mathrm{CH}_{\mathrm{Ar}}}$ ), 124.7 (minor $\mathrm{C}_{\beta}$ ), 124.6 (major $\mathrm{C}_{\beta}$ ), 120.0 (major $\mathrm{CH}_{\mathrm{Ar}}$ ), 119.9 (minor $\mathrm{CH}_{\mathrm{Ar}}$ ), 67.6 (minor $\mathrm{NCO}_{2}-\underline{\mathrm{C}} \mathrm{H}_{2}-\mathrm{CH}$ ), 67.5 (major $\mathrm{NCO}_{2}-\underline{\mathrm{CH}_{2}-}$ $\overline{\mathrm{CH}}$ ), 67.1 (major $\left.\underline{\mathrm{C}}_{2} \mathrm{Ph}\right), 67.0$ (minor $\left.\underline{\mathrm{CH}}_{2} \mathrm{Ph}\right), 66.7$ (major $\mathrm{C}_{\alpha}$ ), 
66.3 (minor $\mathrm{C}_{\alpha}$ ), 54.0 (minor $\mathrm{C}_{\delta}$ ), 53.4 (major $\mathrm{C}_{\delta}$ ), 47.2 (minor $\mathrm{NCO}_{2}-\mathrm{CH}_{2}-\underline{\mathrm{CH}}$ ), 47.1 (major $\mathrm{NCO}_{2}-\mathrm{CH}_{2}-\underline{\mathrm{CH}}$ ) ppm; $R_{f} 0.32$ (hexane/EtOAc 80:20); $[\alpha]_{\mathrm{D}}^{22}-184.1\left(\mathrm{c} 1.2, \mathrm{CHCl}_{3}\right)$; MS (ESI) $(\mathrm{m} /$ z) $426.3[\mathrm{M}+\mathrm{H}]^{+}$, 448.3 $[\mathrm{M}+\mathrm{Na}]^{+}$; HRMS (ESI) for $\mathrm{C}_{27} \mathrm{H}_{23} \mathrm{NNaO}_{4}[\mathrm{M}+\mathrm{Na}]^{+}$calcd for 448.1519, found 448.1524; IR 1750 (s), 1705 (s), 1450 (m), 1415 (s), 1172 (s), 1122 (s), 1105 (s), $734(\mathrm{~s}) \mathrm{cm}^{-1}$.

$\mathrm{N}$-(tert-Butoxycarbonyl)-(2S,3R,4S)-3,4-epoxyproline Benzyl Ester (10a) and $N$-(tert-Butoxycarbonyl)-(2S,3S,4R)-3,4-epoxyproline Benzyl Ester (9a) (Scheme 4). To a solution of alkene 25a $(32.0 \mathrm{~g}, 105.5 \mathrm{mmol})$ in 1,2-dichloroethane $(250 \mathrm{~mL})$ was added meta-chloroperoxybenzoic acid ( $\leq 77 \%$ pure, $30.7 \mathrm{~g}, 137.1 \mathrm{mmol}$ ), and the mixture was refluxed at $90{ }^{\circ} \mathrm{C}$. After $24 \mathrm{~h}$, the mixture was cooled to room temperature and quenched with a saturated aqueous solution of $\mathrm{Na}_{2} \mathrm{~S}_{2} \mathrm{O}_{3}(100 \mathrm{~mL})$. The phases were separated, and the organic layer was washed with a saturated aqueous solution of $\mathrm{NaHCO}_{3}(100 \mathrm{~mL})$ and brine $(100 \mathrm{~mL})$. The organic layer was then dried over $\mathrm{MgSO}_{4}$ and evaporated in vacuo. Purification by flash chromatography (hexane/EtOAc 85:15 to 75:25) yielded 10a (16.3 g, $52 \%)$ and $9 \mathrm{a}(10.6 \mathrm{~g}, 32 \%)$ as colorless oils.

Data for $\mathrm{N}$-(tert-Butoxycarbonyl)-(2S,3R,4S)-3,4-epoxyproline Benzyl Ester (10a): ${ }^{1} \mathrm{H}$ NMR (400 $\mathrm{MHz}, \mathrm{CDCl}_{3}$ ) (58:42 rotamer ratio) $\delta$ 7.41-7.31 (m, 5H major $\mathrm{Ph}+5 \mathrm{H}$ minor $\mathrm{Ph}), 5.29(\mathrm{~d}, J=$ $12.4 \mathrm{~Hz}, 1 \mathrm{H}$, minor $\left.\mathrm{CHH}^{\prime} \mathrm{Ph}\right), 5.23(\mathrm{~d}, J=12.3 \mathrm{~Hz}, 1 \mathrm{H}$, major $\left.\mathrm{CH}^{\prime} \mathrm{Ph}\right), 5.20\left(\mathrm{~d}, J=12.3 \mathrm{~Hz}, 1 \mathrm{H}\right.$, major $\left.\mathrm{CH} \underline{H}^{\prime} \mathrm{Ph}\right), 5.15(\mathrm{~d}, J=$ $12.4 \mathrm{~Hz}, 1 \mathrm{H}$, minor $\left.\mathrm{CH}^{\prime} \mathrm{Ph}\right), 4.72\left(\mathrm{~s}, 1 \mathrm{H}\right.$, minor $\left.\mathrm{C}_{\alpha} \mathrm{H}\right), 4.57(\mathrm{~s}, 1 \mathrm{H}$, major $\left.\mathrm{C}_{\alpha} \mathrm{H}\right), 3.88\left(\mathrm{~d}, J=12.5 \mathrm{~Hz}, 1 \mathrm{H}\right.$, major $\left.\mathrm{C}_{\delta} \underline{\mathrm{H}}^{\prime}\right), 3.82(\mathrm{~d}, J=$ $12.5 \mathrm{~Hz}, 1 \mathrm{H}$, minor $\left.\mathrm{C}_{\delta} \underline{\mathrm{H}} \mathrm{H}^{\prime}\right), 3.75(\mathrm{dd}, J=2.9,0.5 \mathrm{~Hz}, 1 \mathrm{H}$, major $\left.\mathrm{C}_{\beta} \mathrm{H}\right), 3.74\left(\mathrm{dd}, J=2.9,0.4 \mathrm{~Hz}, 1 \mathrm{H}\right.$, minor $\left.\mathrm{C}_{\beta} \mathrm{H}\right), 3.68(\mathrm{ddd}, J=2.9$, $1.3,0.3 \mathrm{~Hz}, 1 \mathrm{H}$, major $\left.\mathrm{C}_{\gamma} \mathrm{H}\right), 3.65(\mathrm{ddd}, J=2.9,1.2,0.4 \mathrm{~Hz}, 1 \mathrm{H}$, minor $\left.\mathrm{C}_{\gamma} \mathrm{H}\right), 3.51\left(\mathrm{dd}, J=12.5,1.4 \mathrm{~Hz}\right.$, major $\left.\mathrm{C}_{\delta} \mathrm{H}^{\prime}\right), 3.48(\mathrm{dd}, J=$ $12.5,1.4 \mathrm{~Hz}$, minor $\left.\mathrm{C}_{\delta} \mathrm{H}^{\prime}\right), 1.45\left(\mathrm{~s}, 9 \mathrm{H}\right.$, minor $\left.\mathrm{CO}_{2} \mathrm{C}\left(\mathrm{C}_{3}\right)_{3}\right), 1.33$ (s, 9H, major $\left.\mathrm{CO}_{2} \mathrm{C}\left(\overline{\mathrm{CH}}_{3}\right)_{3}\right)$ ppm; ${ }^{13} \mathrm{C}\left\{{ }^{1} \mathrm{H}\right\}$ NMR $(100 \mathrm{MHz}$, $\mathrm{CDCl}_{3}$ ) (58:42 rotamer ratio) $\delta 169.2$ (major $\mathrm{C}_{\alpha}-\mathrm{CO}_{2}$ ), 169.1 (minor $\left.\mathrm{C}_{\alpha}-\mathrm{CO}_{2}\right), 154.4\left(\right.$ minor $\left.\mathrm{CO}_{2} \mathrm{C}\left(\mathrm{CH}_{3}\right)_{3}\right), 153.9$ (major $\left.\mathrm{CO}_{2} \mathrm{C}\left(\mathrm{CH}_{3}\right)_{3}\right)$, 135.2 (minor $\underline{\mathrm{C}}_{\mathrm{q}, \mathrm{Ph}}$ ), 135.1 (major $\underline{\mathrm{C}}_{\mathrm{q}, \mathrm{Ph}}$ ), $128.7+128.61+128.59+$ $128.4+128.1$ (major and minor overlap, $\underline{\mathrm{CH}}_{\mathrm{Ph}}$ ), 80.60 (major $\left.\mathrm{CO}_{2} \underline{\mathrm{C}}\left(\mathrm{CH}_{3}\right)_{3}\right), 80.58\left(\right.$ minor $\left.\mathrm{CO}_{2} \underline{\mathrm{C}}\left(\mathrm{CH}_{3}\right)_{3}\right), 67.27\left(\right.$ minor $\left.\underline{\mathrm{C}} \mathrm{H}_{2} \mathrm{Ph}\right)$, 67.25 (major $\mathrm{CH}_{2} \mathrm{Ph}$ ), 60.8 (major $\mathrm{C}_{\alpha}$ ), 60.4 (minor $\mathrm{C}_{\alpha}$ ), 57.3 (major $\mathrm{C}_{\beta}$ ), $56.6\left(\right.$ minor $\mathrm{C}_{\beta}$ ), 54.9 (minor $\mathrm{C}_{\gamma}$ ), 54.5 (major $\mathrm{C}_{\gamma}$ ), 47.2 (minor $\mathrm{C}_{\delta}$ ), 46.8 (major $\mathrm{C}_{\delta}$ ), 28.3 (minor $\mathrm{CO}_{2} \mathrm{C}\left(\mathrm{CH}_{3}\right)_{3}$ ), 28.1 (major $\left.\mathrm{CO}_{2} \mathrm{C}\left(\mathrm{CH}_{3}\right)_{3}\right)$ ppm; $R_{f} 0.35$ (hexane/acetone 80:20); $[\alpha]_{\mathrm{D}}^{22}-45(c$ 1.1, $\left.\mathrm{CHCl}_{3}\right)$; MS (ESI) $(m / z) 342.4[\mathrm{M}+\mathrm{Na}]^{+}$; HRMS (ESI) for $\mathrm{C}_{17} \mathrm{H}_{22} \mathrm{NO}_{5}[\mathrm{M}+\mathrm{H}]^{+}$calcd for 320.1492, found 320.1487; IR 2977 $(\mathrm{w}), 2361(\mathrm{w}), 1750$ (s), 1703 (s), $1416(\mathrm{~m}), 1389(\mathrm{~m}), 1170$ (s) $\mathrm{cm}^{-1}$.

Data for N-(tert-Butoxycarbonyl)-(2S,3S,4R)-3,4-epoxyproline Benzyl Ester (9a): ${ }^{1} \mathrm{H}$ NMR (400 MHz, $\left.\mathrm{CDCl}_{3}\right)$ (60:40 rotamer ratio) $\delta 7.45-7.30(\mathrm{~m}, 5 \mathrm{H}$ major $\mathrm{Ph}+5 \mathrm{H}$ minor $\mathrm{Ph}), 5.35(\mathrm{~d}, J=$ $12.5 \mathrm{~Hz}, 1 \mathrm{H}$, minor $\left.\mathrm{CHH}^{\prime} \mathrm{Ph}\right), 5.27(\mathrm{~d}, J=12.5 \mathrm{~Hz}, 1 \mathrm{H}$, major $\left.\mathrm{CH}^{\prime} \mathrm{Ph}\right), 5.22\left(\mathrm{~d}, J=12.5 \mathrm{~Hz}, 1 \mathrm{H}\right.$, major $\left.\mathrm{CH} \underline{H}^{\prime} \mathrm{Ph}\right), 5.17(\mathrm{~d}, J=$ $12.5 \mathrm{~Hz}, 1 \mathrm{H}$, minor $\left.\mathrm{CH}^{\prime} \mathrm{Ph}\right), 4.45\left(\mathrm{~d}, J=1.6 \mathrm{~Hz}, 1 \mathrm{H}\right.$, minor $\left.\mathrm{C}_{\alpha} \mathrm{H}\right)$, $4.37\left(\mathrm{~d}, J=1.8 \mathrm{~Hz}, 1 \mathrm{H}\right.$, major $\mathrm{C}_{\alpha} \mathrm{H}$ ), 3.95 (app. t, $J=2.5 \mathrm{~Hz}, 1 \mathrm{H}$ major $\mathrm{C}_{\beta} \mathrm{H}+1 \mathrm{H}$ minor $\left.\mathrm{C}_{\beta} \mathrm{H}\right), 3.86(\mathrm{~d}, J=12.7 \mathrm{~Hz}, 1 \mathrm{H}$, major $\left.\mathrm{C}_{\delta} \mathrm{HH}^{\prime}\right), 3.82\left(\mathrm{~d}, J=12.7 \mathrm{~Hz}, 1 \mathrm{H}\right.$, minor $\left.\mathrm{C}_{\delta} \underline{\mathrm{H}}^{\prime}\right), 3.79-3.74(\mathrm{~m}, 1 \mathrm{H}$ major $\mathrm{C}_{\gamma} \mathrm{H}+1 \mathrm{H}$ minor $\left.\mathrm{C}_{\gamma} \mathrm{H}\right), 3.56(\mathrm{br} \mathrm{dd}, J=12.7,1.2 \mathrm{~Hz}$, major $\left.\mathrm{C}_{\delta} \mathrm{H}^{\prime}\right), 3.51$ (br dd, $J=12.7,1.2 \mathrm{~Hz}$, minor $\left.\mathrm{C}_{\delta} \mathrm{H}_{\mathrm{H}^{\prime}}\right), 1.45(\mathrm{~s}, 9 \mathrm{H}$, minor $\left.\mathrm{CO}_{2} \mathrm{C}\left(\mathrm{C}_{3}\right)_{3}\right), 1.33$ (s, 9H, major $\left.\mathrm{CO}_{2} \mathrm{C}\left(\mathrm{CH}_{3}\right)_{3}\right)$ ppm; ${ }^{13} \mathrm{C}\left\{{ }^{1} \mathrm{H}\right\}$ NMR $\left(100 \mathrm{MHz}, \mathrm{CDCl}_{3}\right)\left(60: 40\right.$ ratio) $\delta 167.9$ (major $\mathrm{C}_{\alpha^{-}}$ $\underline{\mathrm{CO}_{2}}$ ), 167.5 (minor $\mathrm{C}_{\alpha}-\underline{\mathrm{CO}} \mathrm{O}_{2}$ ), 154.3 (minor $\left.\underline{\mathrm{CO}}_{2} \mathrm{C}\left(\mathrm{CH}_{3}\right)_{3}\right), 153.8$ (major $\underline{\mathrm{CO}}_{2} \mathrm{C}\left(\mathrm{CH}_{3}\right)_{3}$ ), 135.6 (minor $\underline{\mathrm{C}}_{\mathrm{q}, \mathrm{Ph}}$ ), 135.4 (major $\underline{\mathrm{C}}_{\mathrm{q}, \mathrm{Ph}}$ ), $128.8+128.6+128.51+128.48+128.41+128.2$ (major and minor overlap, $\underline{\mathrm{C}} \mathrm{H}_{\mathrm{Ph}}$ ), 80.8 (major $\left.\mathrm{CO}_{2} \underline{\mathrm{C}}\left(\mathrm{CH}_{3}\right)_{3}\right), 80.6$ (minor $\mathrm{CO}_{2} \mathrm{C}$ $\left.\left(\mathrm{CH}_{3}\right)_{3}\right), 67.2\left(2 \mathrm{C}\right.$, major $\mathrm{CH}_{2} \mathrm{Ph}+$ minor $\left.\underline{\mathrm{CH}}_{2} \mathrm{Ph}\right), 60.2$ (major $\mathrm{C}_{\alpha}$ ), $60.1\left(\right.$ minor $\left.\mathrm{C}_{\alpha}\right), 58.0\left(\right.$ major $\left.\mathrm{C}_{\beta}\right), 57.3\left(\right.$ minor $\left.\mathrm{C}_{\beta}\right), 56.1$ (minor $\mathrm{C}_{\gamma}$ ), 55.6 (major $\mathrm{C}_{\gamma}$ ), $48.0\left(\right.$ minor $\mathrm{C}_{\delta}$ ), $47.8\left(\right.$ major $\mathrm{C}_{\delta}$ ), 28.3 (minor $\left.\mathrm{CO}_{2} \mathrm{C}\left(\mathrm{CH}_{3}\right)_{3}\right), 28.1$ (major $\left.\mathrm{CO}_{2} \mathrm{C}\left(\underline{\mathrm{CH}}_{3}\right)_{3}\right)$ ppm; $R_{f} 0.25$ (hexane/ acetone 80:20); $[\alpha]_{\mathrm{D}}^{22}-53\left(\mathrm{c} 1.3, \mathrm{CHCl}_{3}\right) ; \mathrm{MS}(\mathrm{ESI})(\mathrm{m} / \mathrm{z}) 320.5[\mathrm{M}$ $+\mathrm{H}]^{+}, 342.4[\mathrm{M}+\mathrm{Na}]^{+}$; HRMS (ESI) for $\mathrm{C}_{17} \mathrm{H}_{22} \mathrm{NO}_{5}[\mathrm{M}+\mathrm{H}]^{+}$ calcd for 320.1492, found 320.1491; IR 2977 (w), 2361 (w), 1761 (s), $1703(\mathrm{~s}), 1379(\mathrm{~m}), 1170(\mathrm{~s}) \mathrm{cm}^{-1}$.

( \pm )- $N$-(Acetyl)-(2S,3R,4S)-3,4-epoxyproline Benzyl Ester (10) and ( \pm )- $N$-(Acetyl)-(2S,3S,4R)-3,4-epoxyproline Benzyl Ester (9b) (Scheme 4). Alkene ( \pm )-25b (16.2 g, $66.2 \mathrm{mmol})$ was dissolved in 1,2-dichloroethane $(250 \mathrm{~mL})$, meta-chloroperoxybenzoic acid ( $<77 \%$ pure, $34.3 \mathrm{~g}, 198.5 \mathrm{mmol}$ ) was added, and the mixture was refluxed at $70{ }^{\circ} \mathrm{C}$. TLC analysis indicated the reaction was finished after $25 \mathrm{~h}$. Next, the reaction mixture was cooled to room temperature and washed with a saturated aqueous solution of $\mathrm{Na}_{2} \mathrm{~S}_{2} \mathrm{O}_{3}(250 \mathrm{~mL})$, a saturated aqueous solution of $\mathrm{NaHCO}_{3}(250$ $\mathrm{mL})$, and brine $(250 \mathrm{~mL})$. The organic layer was dried over $\mathrm{MgSO}_{4}$ and evaporated in vacuo. Purification by flash chromatography (hexane/acetone 60:40 to $40: 60)$ yielded $10 b$ (9.42 g, 55\%) and $9 \mathbf{b}$ (4.26 g, 25\%) as slightly yellow/off-white solids. A sample of $9 \mathrm{~b}$ was recrystallized from DCM and submitted for X-ray analysis.

Data for ( \pm )-N-(Acetyl)-(2S,3R,4S)-3,4-epoxyproline Benzyl Ester (10b): ${ }^{1} \mathrm{H}$ NMR (400 MHz, $\mathrm{CDCl}_{3}$ ) (69:31 rotamer ratio) $\delta 7.42-$ $7.30(\mathrm{~m}, 5 \mathrm{H}$ major $\mathrm{Ph}+5 \mathrm{H}$ minor $\mathrm{Ph}), 5.26(\mathrm{~d}, J=12.2 \mathrm{~Hz}, 1 \mathrm{H}$, minor $\left.\mathrm{CH}^{\prime} \mathrm{Ph}\right), 5.24\left(\mathrm{~d}, J=12.3 \mathrm{~Hz}, 1 \mathrm{H}\right.$, major $\left.\mathrm{CHH}^{\prime} \mathrm{Ph}\right), 5.22(\mathrm{~d}$, $J=12.2 \mathrm{~Hz}, 1 \mathrm{H}$, minor $\left.\mathrm{CH}^{\prime} \mathrm{Ph}\right), 5.18(\mathrm{~d}, J=12.3 \mathrm{~Hz}, 1 \mathrm{H}$, major $\left.\mathrm{CH}^{\prime} \mathrm{Ph}\right), 4.90\left(\mathrm{~s}, 1 \mathrm{H}\right.$, major $\left.\mathrm{C}_{\alpha} \underline{\mathrm{H}}\right), 4.60\left(\mathrm{~s}, 1 \mathrm{H}\right.$, minor $\left.\mathrm{C}_{\alpha} \underline{\mathrm{H}}\right), 4.14$ $\left(\mathrm{d}, J=13.6 \mathrm{~Hz}, 1 \mathrm{H}\right.$, minor $\left.\mathrm{C}_{\delta} \mathrm{HH}^{\prime}\right), 3.87(\mathrm{~d}, J=2.9 \mathrm{~Hz}, 1 \mathrm{H}$, minor $\left.\mathrm{C}_{\beta} \underline{\mathrm{H}}\right), 3.84\left(\mathrm{~d}, J=11.6 \mathrm{~Hz}, 1 \mathrm{H}\right.$, major $\left.\mathrm{C}_{\delta} \underline{\mathrm{H}} \mathrm{H}^{\prime}\right), 3.77(\mathrm{~d}, J=2.9 \mathrm{~Hz}$, $1 \mathrm{H}$, major $\left.\mathrm{C}_{\beta} \underline{\mathrm{H}}\right), 3.72\left(\mathrm{~m}, 1 \mathrm{H}\right.$ major $\mathrm{C}_{\gamma} \underline{\mathrm{H}}+1 \mathrm{H}$ minor $\left.\mathrm{C}_{\gamma} \underline{\mathrm{H}}\right), 3.71(\mathrm{~d}$, $J=11.6 \mathrm{~Hz}, 1 \mathrm{H}$, major $\left.\mathrm{C}_{\delta} \mathrm{H}^{\prime}\right), 3.43(\mathrm{~d}, J=13.5 \mathrm{~Hz}, 1 \mathrm{H}$, minor $\left.\mathrm{C}_{\delta} \mathrm{H}_{\mathrm{H}^{\prime}}\right), 2.05$ (s, $3 \mathrm{H}$, major $\left.\mathrm{C}_{3}\right), 1.92$ (s, 3H, minor $\left.\mathrm{C}_{3}\right)$ ppm; ${ }^{13} \mathrm{C}\left\{{ }^{1} \mathrm{H}\right\}$ NMR $\left(100 \mathrm{MHz}, \mathrm{CDCl}_{3}\right)(69: 31$ rotamer ratio) $\delta 170.3$ (minor $\left.\mathrm{N}-\mathrm{COCH}_{3}\right), 170.2$ (major $\left.\mathrm{N}-\mathrm{COCH}_{3}\right), 168.4$ (major $\mathrm{C}_{\alpha}{ }^{-}$ $\underline{\mathrm{CO}}_{2}$ ), 168.2 (minor $\mathrm{C}_{\alpha}-\underline{\mathrm{CO}}_{2}$ ), 135.1 (major $\underline{\mathrm{C}}_{\mathrm{q}, \mathrm{Ph}}$ ), 134.6 (minor $\underline{\mathrm{C}}_{\mathrm{q}, \mathrm{Ph}}$ ), $128.9+128.8+128.6+128.5+128.4+128.1$ (major and minor overlap, $\underline{\mathrm{CH}}_{\mathrm{Ph}}$ ), 67.9 (minor $\underline{\mathrm{CH}}_{2} \mathrm{Ph}$ ), 67.4 (major $\underline{\mathrm{CH}}_{2} \mathrm{Ph}$ ), 61.6 (minor $\underline{\mathrm{C}}_{\alpha} \mathrm{H}$ ), 59.9 (major $\underline{\mathrm{C}}_{\alpha} \mathrm{H}$ ), 57.2 (minor $\underline{\mathrm{C}}_{\beta} \mathrm{H}$ ), 56.3 (major $\underline{\mathrm{C}}_{\beta} \mathrm{H}$ ), $54.9\left(\right.$ major $\left.\underline{\mathrm{C}}_{\gamma} \mathrm{H}\right), 53.8$ (minor $\left.\underline{\mathrm{C}}_{\gamma} \mathrm{H}\right), 48.3$ (major $\left.\underline{\mathrm{C}}_{\delta} \mathrm{H}_{2}\right), 46.5\left(\right.$ minor $\left.\underline{\mathrm{C}}_{\delta} \mathrm{H}_{2}\right), 22.1\left(\right.$ minor $\left.\underline{\mathrm{CH}}_{3}\right), 22.0$ (major $\left.\underline{\mathrm{CH}}_{3}\right)$ ppm; mp 70-72 ${ }^{\circ} \mathrm{C} ; R_{f} 0.38$ (hexane/acetone 60:40); MS (ESI) $(\mathrm{m} /$ z) $262.3[\mathrm{M}+\mathrm{H}]^{+}, 284.3[\mathrm{M}+\mathrm{Na}]^{+}$; HRMS (ESI) for $\mathrm{C}_{14} \mathrm{H}_{16} \mathrm{NO}_{4}$ $[\mathrm{M}+\mathrm{H}]^{+}$calcd for 262.1074, found 262.1067; IR 1747 (s), 1652 (s), $1213(\mathrm{~s}), 1175(\mathrm{~s}) \mathrm{cm}^{-1}$.

Data for ( \pm )-N-(Acetyl)-(2S,3S,4R)-3,4-epoxyproline Benzyl Ester (9b): ${ }^{1} \mathrm{H}$ NMR $\left(400 \mathrm{MHz}, \mathrm{CDCl}_{3}\right)$ (80:20 rotamer ratio) $\delta 7.41-$ $7.30(\mathrm{~m}, 5 \mathrm{H}$ major $\mathrm{Ph}+5 \mathrm{H}$ minor $\mathrm{Ph}), 5.28\left(\mathrm{~s}, 2 \mathrm{H}\right.$, minor $\left.\mathrm{C}_{2} \mathrm{Ph}\right)$, $5.25\left(\mathrm{~s}, 2 \mathrm{H}\right.$, major $\left.\mathrm{C}_{2} \mathrm{Ph}\right), 4.56\left(\mathrm{~d}, J=2.3 \mathrm{~Hz}, 1 \mathrm{H}\right.$, major $\left.\mathrm{C}_{\alpha} \underline{\mathrm{H}}\right), 4.55$ $\left(\mathrm{d}, J=2.8 \mathrm{~Hz}, 1 \mathrm{H}\right.$, minor $\left.\mathrm{C}_{\alpha} \underline{\mathrm{H}}\right), 4.12\left(\mathrm{t}, J=2.8 \mathrm{~Hz}, 1 \mathrm{H}\right.$, minor $\left.\mathrm{C}_{\beta} \underline{\mathrm{H}}\right)$, $3.98\left(\mathrm{t}, J=2.6 \mathrm{~Hz}, 1 \mathrm{H}\right.$, major $\left.\mathrm{C}_{\beta} \underline{\mathrm{H}}\right), 3.94(\mathrm{~d}, J=11.7 \mathrm{~Hz}, 1 \mathrm{H}$, major $\left.\mathrm{C}_{\delta} \underline{\mathrm{HH}^{\prime}}\right), 3.87\left(\mathrm{~d}, J=13.9 \mathrm{~Hz}, 1 \mathrm{H}\right.$, minor $\left.\mathrm{C}_{\delta} \underline{\mathrm{H}} \mathrm{H}^{\prime}\right), 3.86(\mathrm{dd}, J=2.9$, $2.1 \mathrm{~Hz}, 1 \mathrm{H}$ major $\left.\mathrm{C}_{\gamma} \underline{\mathrm{H}}\right), 3.83\left(\mathrm{dd}, J=2.8,2.3 \mathrm{~Hz}, 1 \mathrm{H}\right.$ minor $\left.\mathrm{C}_{\gamma} \underline{\mathrm{H}}\right)$, $3.72\left(\mathrm{dd}, J=13.9,2.1 \mathrm{~Hz}, 1 \mathrm{H}\right.$, minor $\left.\mathrm{C}_{\delta} \mathrm{HH}^{\prime}\right), 3.87(\mathrm{dd}, J=11.7,2.0$ $\mathrm{Hz}, 1 \mathrm{H}$, major $\left.\mathrm{C}_{\delta} \mathrm{H}^{\prime}\right), 2.06$ (s, $3 \mathrm{H}$, major $\left.\mathrm{CH}_{3}\right), 1.86(\mathrm{~s}, 3 \mathrm{H}$, minor $\left.\mathrm{CH}_{3}\right)$ ppm; ${ }^{13} \mathrm{C}\left\{{ }^{1} \mathrm{H}\right\}$ NMR $\left(100 \mathrm{MHz}, \mathrm{CDCl}_{3}\right)(80: 20$ rotamer ratio) $\delta 170.5$ (minor $\mathrm{N}-\mathrm{COCH}_{3}$ ), 170.1 (major $\mathrm{N}-\mathrm{COCH}_{3}$ ), 167.5 (minor $\mathrm{C}_{\alpha}-\mathrm{CO}_{2}$ ), 166.6 (major $\mathrm{C}_{\alpha}-\mathrm{CO}_{2}$ ), 135.5 (major $\underline{\mathrm{C}}_{\mathrm{q}, \mathrm{Ph}}$ ), 134.9 (minor $\left.\underline{\mathrm{C}}_{\mathrm{q}, \mathrm{Ph}}\right), 128.7+128.5+128.4+128.2+128.1$ (major and minor overlap, $\underline{\mathrm{C}} \mathrm{H}_{\mathrm{Ph}}$ ), 67.8 (minor $\left.\underline{\mathrm{CH}}_{2} \mathrm{Ph}\right), 67.2\left(\right.$ major $\left.\underline{\mathrm{CH}}_{2} \mathrm{Ph}\right), 61.1$ (minor $\underline{\mathrm{C}}_{\alpha} \mathrm{H}$ ), 59.8 (major $\underline{\mathrm{C}}_{\alpha} \mathrm{H}$ ), 59.1 (minor $\underline{\mathrm{C}}_{\beta} \mathrm{H}$ ), 56.9 (major $\left.\underline{\mathrm{C}}_{\beta} \mathrm{H}\right), 56.2$ (major $\left.\underline{\mathrm{C}}_{\gamma} \mathrm{H}\right), 55.8$ (minor $\left.\underline{\mathrm{C}}_{\gamma} \mathrm{H}\right), 48.8\left(\right.$ major $\left.\underline{\mathrm{C}}_{\delta} \mathrm{H}_{2}\right), 48.3$ (minor $\left.\underline{\mathrm{C}}_{\delta} \mathrm{H}_{2}\right), 21.8$ (major $\left.\mathrm{CH}_{3}\right), 21.3$ (minor $\left.\mathrm{CH}_{3}\right)$ ppm; mp 96$100{ }^{\circ} \mathrm{C} ; R_{f} 0.15$ (hexane/acetone 60:40); MS (ESI) $(\mathrm{m} / z) 262.2[\mathrm{M}$ $+\mathrm{H}]^{+}, 284.3[\mathrm{M}+\mathrm{Na}]^{+}$; HRMS (ESI) for $\mathrm{C}_{14} \mathrm{H}_{16} \mathrm{NO}_{4}[\mathrm{M}+\mathrm{H}]^{+}$ calcd for 262.1074, found 262.1069; IR 1756 (s), 1650 (s), 1170 (s) $\mathrm{cm}^{-1}$.

Benzyl 1H-Pyrrole-2-carboxylate (35) (Table 1, Entry 1). Epoxide 10a $(150.0 \mathrm{mg}, 0.470 \mathrm{mmol})$ was dissolved in $\mathrm{Et}_{3} \mathrm{~N} \cdot 3 \mathrm{HF}$, and the mixture was stirred at $90^{\circ} \mathrm{C}$. After $24 \mathrm{~h}$, no reaction was observed and the temperature was increased to $130{ }^{\circ} \mathrm{C}$. Overnight, a white/ brown gel formed. Next, the mixture was poured in a saturated aqueous solution of $\mathrm{NaHCO}_{3}(120 \mathrm{~mL})$, and the aqueous layer was extracted with $\mathrm{Et}_{2} \mathrm{O}(3 \times 200 \mathrm{~mL})$. The combined organic phases were dried over $\mathrm{MgSO}_{4}$ and evaporated in vacuo to yield $120.3 \mathrm{mg}$ of crude product. NMR analysis of the crude product allowed us to 
conclude that pyrrole 35 was the only product: ${ }^{1} \mathrm{H}$ NMR $(500 \mathrm{MHz}$, $\left.\mathrm{CDCl}_{3}\right) \delta 9.30(\mathrm{br} \mathrm{s}, 1 \mathrm{H}, \mathrm{N} \underline{\mathrm{H}}), 7.46-7.32(\mathrm{~m}, 5 \mathrm{H}, \mathrm{C} \underline{\mathrm{H}} \mathrm{Ar}), 6.99$ $\left(\mathrm{ddd}, J=3.8,2.4,1.5 \mathrm{~Hz}, 1 \mathrm{H}, \mathrm{C}_{\delta} \mathrm{H}\right), 6.96(\mathrm{td}, J=2.7,1.5 \mathrm{~Hz}, 1 \mathrm{H}$, $\left.\mathrm{C}_{\beta} \mathrm{H}\right), 6.28\left(\mathrm{dt}, J=3.8,2.5 \mathrm{~Hz}, 1 \mathrm{H}, \mathrm{C}_{\gamma} \mathrm{H}\right), 5.33\left(\mathrm{~s}, 2 \mathrm{H}, \mathrm{C}_{2} \mathrm{Ph}\right) \mathrm{ppm}$; ${ }^{13} \mathrm{C}\left\{{ }^{1} \mathrm{H}\right\} \operatorname{NMR}\left(126 \mathrm{MHz}, \mathrm{CDCl}_{3}\right) \delta 161.0\left(\mathrm{C}_{\alpha}-\underline{C O}_{2}\right), 136.1\left(\underline{\mathrm{C}}_{\mathrm{q}, \mathrm{Ph}}\right)$, $128.6\left(2 \times \underline{\mathrm{CH}}_{\mathrm{Ar}}\right), 128.2\left(\underline{\mathrm{CH}}_{\mathrm{Ar}}\right), 128.1\left(2 \times \underline{\mathrm{CH}}_{\mathrm{Ar}}\right), 123.1\left(\mathrm{C}_{\beta}\right)$, $122.6\left(\mathrm{C}_{\alpha}\right), 115.6\left(\mathrm{C}_{\delta}\right), 110.5\left(\mathrm{C}_{\gamma}\right), 66.0\left(\underline{\mathrm{CH}}_{2} \mathrm{Ph}\right) \mathrm{ppm} ; R_{f} 0.44$ (hexane/acetone 80:20); MS (ESI) $(m / z) 202.1[\mathrm{M}+\mathrm{H}]^{+}$; HRMS (ESI) for $\mathrm{C}_{12} \mathrm{H}_{11} \mathrm{NNaO}_{2}[\mathrm{M}+\mathrm{Na}]^{+}$calcd for 224.0682, found 224.0678; IR 3413 (br m), 3312 (br m), 2360 (m), 2340 (m), 1680 (s), $1410(\mathrm{~s}), 1304(\mathrm{~s}), 1156(\mathrm{~m}), 1124(\mathrm{~s}) \mathrm{cm}^{-1}$. Chemical shift data correspond to literature data. ${ }^{84}$

$( \pm)-N$-(Acetyl)-(2S,3R,4R)-3-hydroxy-4-fluoroproline Benzyl Ester (33b) (Table 1, Entry 5). DMPU.HF $(0.4 \mathrm{~mL})$ was added dropwise to a solution of epoxide $( \pm)-10 b(130.0 \mathrm{mg}, 0.498 \mathrm{mmol})$ in DCM $(3.0 \mathrm{~mL})$, and the mixture was stirred at room temperature. Within $1 \mathrm{~h}$, a white gel had formed in the reaction mixture. TLC analysis after $19 \mathrm{~h}$ indicated the presence of the starting material, upon which the reaction temperature was increased to $50^{\circ} \mathrm{C}$. After an additional $24 \mathrm{~h}$, the reaction mixture was quenched with a saturated aqueous solution of $\mathrm{NaHCO}_{3}(8 \mathrm{~mL})$ and the mixture was stirred over basic $\mathrm{Al}_{2} \mathrm{O}_{3}$ for $10 \mathrm{~min}$. After removal of the basic alumina via filtration, the aqueous layer was extracted with DCM $(3 \times 10 \mathrm{~mL})$. The combined organic phases were washed with brine $(1 \times 10 \mathrm{~mL})$, dried over $\mathrm{MgSO}_{4}$, and evaporated in vacuo. The crude product was purified via HPLC (hexane/acetone 60:40) to yield recovered the starting material $\mathbf{1 0 b}(38.5 \mathrm{mg}, 30 \%)$ and $33 \mathbf{b}$ as a clear oil $(21.1 \mathrm{mg}$, $15 \%)$, along with a trace amount of DMPU $(<3 \%)$.

Data for ( \pm )- $N$-(Acetyl)-(2S,3R,4R)-3-hydroxy-4-fluoroproline Benzyl Ester (33b): ${ }^{1} \mathrm{H}$ NMR (400 $\left.\mathrm{MHz}, \mathrm{CDCl}_{3}\right)$ (67:33 rotamer ratio) $\delta 7.42-7.28(\mathrm{~m}, 5 \mathrm{H}$ major $\mathrm{Ph}+5 \mathrm{H}$ minor $\mathrm{Ph}), 5.25$ $\left(\mathrm{d}, J=12.1 \mathrm{~Hz}, 1 \mathrm{H}\right.$, minor $\left.\mathrm{C}_{\underline{H}} \mathrm{H}^{\prime} \mathrm{Ph}\right), 5.19(\mathrm{~d}, J=12.4 \mathrm{~Hz}, 1 \mathrm{H}$, major $\left.\mathrm{CH}^{\prime} \mathrm{Ph}\right), 5.16\left(\mathrm{~d}, J=12.1 \mathrm{~Hz}, 1 \mathrm{H}\right.$, minor $\left.\mathrm{CH} \underline{H}^{\prime} \mathrm{Ph}\right), 5.12(\mathrm{~d}, J=$ $12.2 \mathrm{~Hz}, 1 \mathrm{H}$, major $\left.\mathrm{CH}^{\prime} \mathrm{Ph}\right), 5.00(\mathrm{dd}, J=50.1,3.9 \mathrm{~Hz}, 1 \mathrm{H}$, major $\left.\mathrm{C}_{\gamma} \mathrm{H}\right), 4.92\left(\mathrm{dd}, J=49.9,3.3 \mathrm{~Hz}, 1 \mathrm{H}\right.$, minor $\left.\mathrm{C}_{\gamma} \mathrm{H}\right), 4.76-4.55(\mathrm{~m}, 1 \mathrm{H}$ major $\mathrm{C}_{\beta} \mathrm{H}+1 \mathrm{H}$ minor $\left.\mathrm{C}_{\beta} \mathrm{H}\right), 4.72\left(\mathrm{~s}, 1 \mathrm{H}\right.$, major $\left.\mathrm{C}_{\alpha} \mathrm{H}\right), 4.45(\mathrm{~s}, 1 \mathrm{H}$, minor $\left.\mathrm{C}_{\alpha} \mathrm{H}\right), 4.33-4.13(\mathrm{~m}, 1 \mathrm{H}$ major $\mathrm{OH}+1 \mathrm{H}$ minor $\mathrm{OH}), 4.03-$ $3.57\left(\mathrm{~m}, 2 \mathrm{H}\right.$ major $\mathrm{C}_{\delta} \mathrm{H}_{2}+2 \mathrm{H}$ minor $\left.\mathrm{C}_{\delta} \mathrm{H}_{2}\right), 2.09$ (s, $3 \mathrm{H}$, major $\left.\mathrm{CH}_{3}\right), 1.96\left(\mathrm{~s}, 3 \mathrm{H}\right.$, minor $\left.\mathrm{CH}_{3}\right)$ ppm; ${ }^{13} \mathrm{C}\left\{{ }^{1} \mathrm{H}\right\}$ NMR $(100 \mathrm{MHz}$, $\mathrm{CDCl}_{3}$ ) (67:33 rotamer ratio) $\delta 171.1$ (minor $\left.\mathrm{N}-\mathrm{COCH}_{3}\right), 170.8$ (major $\mathrm{N}-\mathrm{COCH}_{3}$ ), $168.2\left(\right.$ minor $\mathrm{C}_{\alpha}-\mathrm{CO}_{2}$ ), 167.9 (major $\mathrm{C}_{\alpha}-\mathrm{CO}_{2}$ ), 135.3 (major $\underline{\mathrm{C}}_{\mathrm{q}, \mathrm{Ph}}$ ), 134.9 (minor $\underline{\mathrm{C}}_{\mathrm{q}, \mathrm{Ph}}$ ), $128.64+128.62+128.5+$ $128.4+128.3+128.1$ (major and minor overlap, $\left.\underline{\mathrm{C}}_{\mathrm{Ph}}\right), 94.6(\mathrm{~d}, J=$ $181.2 \mathrm{~Hz}$, major $\left.\mathrm{C}_{\gamma}\right), 93.3\left(\mathrm{~d}, J=179.0 \mathrm{~Hz}\right.$, minor $\left.\mathrm{C}_{\gamma}\right), 77.5(\mathrm{~d}, J=$ $28.6 \mathrm{~Hz}$, minor $\mathrm{C}_{\beta}$ ), $75.6\left(\mathrm{~d}, J=28.6 \mathrm{~Hz}\right.$, major $\mathrm{C}_{\beta}$ ), 67.7 (minor $\mathrm{CH}_{2} \mathrm{Ph}$ ), 67.3 (major $\mathrm{CH}_{2} \mathrm{Ph}$ ), 65.7 (major $\mathrm{C}_{\alpha}$ ), 65.6 (minor $\mathrm{C}_{\alpha}$ ), $52.1\left(\mathrm{~d}, J=23.5 \mathrm{~Hz}\right.$, major $\left.\mathrm{C}_{\delta}\right), 50.9\left(\mathrm{~d}, J=23.5 \mathrm{~Hz}\right.$, minor $\left.\mathrm{C}_{\delta}\right)$, $22.02\left(\right.$ major N-COCH$\left.{ }_{3}\right), 21.98\left(\right.$ minor N-COC $\left.H_{3}\right)$ ppm; ${ }^{19} \mathrm{~F}$ NMR $\left(376 \mathrm{MHz}, \mathrm{CDCl}_{3}\right)(70: 30$ rotamer ratio) $\delta-180.47$ (dddd, $J=49.9$, $39.5,28.6,6.9 \mathrm{~Hz}, 1 \mathrm{~F}$, minor), -182.19 (dddd, $J=49.4,36.4,26.9$, $8.7 \mathrm{~Hz}, 1 \mathrm{~F}$, major) ppm; ${ }^{19} \mathrm{~F}\left\{{ }^{1} \mathrm{H}\right\}$ NMR $\left(376 \mathrm{MHz}, \mathrm{CDCl}_{3}\right)(70: 30$ rotamer ratio) $\delta-180.61$ (s, $1 \mathrm{~F}$, minor), -182.34 (s, 1F major) ppm; $R_{f} 0.22$ (hexane/acetone 60:40); MS (ESI) $(\mathrm{m} / z) 282.4[\mathrm{M}+\mathrm{H}]^{+}$, $304.3[\mathrm{M}+\mathrm{Na}]^{+}$; HRMS (ESI) for $\mathrm{C}_{14} \mathrm{H}_{17} \mathrm{FNO}_{4}[\mathrm{M}+\mathrm{H}]^{+}$calcd for 282.1136, found 282.1135; IR 3278 (br m), 1745 (s), 1626 (s), 1448 (m), $1420(\mathrm{~m}), 1176(\mathrm{~s}) \mathrm{cm}^{-1}$.

( \pm )- $N$-(tert-Butoxycarbonyl)-(2S,3R,4R)-3-hydroxy-4-chloroproline Benzyl Ester (32a) (Table 1, Entry 7). To a solution of epoxide $( \pm)-10 \mathrm{a}(129.8 \mathrm{mg}, 0.406 \mathrm{mmol})$ in DCE $(10 \mathrm{~mL})$ was added $\mathrm{Bu}_{4} \mathrm{NH}_{2} \mathrm{~F}_{3}$ (tech. $90 \%, 0.1 \mathrm{~mL}$ ), and the mixture was stirred at room temperature. After $2.5 \mathrm{~h}$, no reaction was observed and the reaction temperature was increased to $90{ }^{\circ} \mathrm{C}$. TLC analysis indicated complete consumption of the starting material after $26 \mathrm{~h}$. Next, the mixture was diluted with DCM $(3 \mathrm{~mL})$ and water $(3 \mathrm{~mL})$ and cooled to $0{ }^{\circ} \mathrm{C}$. The reaction was quenched with a saturated aqueous solution of $\mathrm{NaHCO}_{3}(5 \mathrm{~mL})$, and the aqueous layer was extracted with DCM $(3 \times 10 \mathrm{~mL})$. The combined organic phases were dried over $\mathrm{MgSO}_{4}$ and evaporated in vacuo. Purification by flash chromatography (hexane/EtOAc 75:25) yielded chlorohydrin 32a as a clear, yellowish oil (106.2 mg, 74\%): ${ }^{1} \mathrm{H}$ NMR (400 $\mathrm{MHz}, \mathrm{CDCl}_{3}$ ) (56:44 rotamer ratio) $\delta$ 7.43-7.29 (m, 5H major $\mathrm{Ph}+5 \mathrm{H}$ minor $\mathrm{Ph}), 5.29(\mathrm{~d}, J=$ $12.4 \mathrm{~Hz}, 1 \mathrm{H}$, minor $\left.\mathrm{CH}^{\prime} \mathrm{Ph}\right), 5.24(\mathrm{~d}, J=12.2 \mathrm{~Hz}, 1 \mathrm{H}$, major $\mathrm{CHH}^{\prime} \mathrm{Ph}$ ), 5.17 (d, $J=12.2 \mathrm{~Hz}, 1 \mathrm{H}$, major $\left.\mathrm{CH}^{\prime}{ }^{\prime} \mathrm{Ph}\right), 5.09(\mathrm{~d}, J=$ $12.4 \mathrm{~Hz}, 1 \mathrm{H}$, minor $\left.\mathrm{CH}^{\prime} \mathrm{Ph}\right), 4.55-4.45\left(\mathrm{~m}, 1 \mathrm{H}\right.$ major $\mathrm{C}_{\beta} \mathrm{H}+1 \mathrm{H}$ minor $\left.\mathrm{C}_{\beta} \mathrm{H}\right), 4.39\left(\mathrm{~d}, J=2.5 \mathrm{~Hz}, 1 \mathrm{H}\right.$, minor $\left.\mathrm{C}_{\alpha} \mathrm{H}\right), 4.26(\mathrm{~d}, J=3.3$ $\mathrm{Hz}, 1 \mathrm{H}$, major $\left.\mathrm{C}_{\alpha} \mathrm{H}\right), 4.18-3.98\left(\mathrm{~m}, 1 \mathrm{H}\right.$ major $\mathrm{C}_{\gamma} \mathrm{H}+1 \mathrm{H}$ minor $\mathrm{C}_{\gamma} \mathrm{H}$ $+1 \mathrm{H}$ major $\mathrm{C}_{\delta} \underline{\mathrm{H}} \mathrm{H}^{\prime}+1 \mathrm{H}$ minor $\left.\mathrm{C}_{\delta} \underline{\mathrm{H}} \mathrm{H}^{\prime}\right)$, 3.69-3.61 (m, $1 \mathrm{H}$ major $\mathrm{C}_{\delta} \mathrm{H}_{\mathrm{H}^{\prime}}+1 \mathrm{H}$ minor $\mathrm{C}_{\delta} \mathrm{HH}^{\prime}$ ), 3.60-3.42 (br m, $1 \mathrm{H}$ major $\mathrm{OH}+1 \mathrm{H}$ minor $\mathrm{OH}), 1.45\left(\mathrm{~s}, 9 \mathrm{H}\right.$, minor $\left.\mathrm{CO}_{2} \mathrm{C}\left(\mathrm{C}_{3}\right)_{3}\right), 1.32$ (s, $9 \mathrm{H}$, major $\left.\mathrm{CO}_{2} \mathrm{C}\left(\mathrm{CH}_{3}\right)_{3}\right)$ ppm; ${ }^{13} \mathrm{C}\left\{{ }^{1} \mathrm{H}\right\}$ NMR $\left(100 \mathrm{MHz}, \mathrm{CDCl}_{3}\right)$ (56:44 rotamer ratio) $\delta 169.9$ (major $\mathrm{C}_{\alpha}-\mathrm{CO}_{2}$ ), 169.4 (minor $\mathrm{C}_{\alpha}-\underline{\mathrm{CO}}_{2}$ ), 154.2 (minor $\mathrm{CO}_{2} \mathrm{C}\left(\mathrm{CH}_{3}\right)_{3}$ ), 153.6 (major $\underline{\mathrm{CO}}_{2} \mathrm{C}\left(\mathrm{CH}_{3}\right)_{3}$ ), 135.3 (minor $\underline{\mathrm{C}}_{\mathrm{q}, \mathrm{Ph}}$ ), $135.2\left(\right.$ major $\left.\underline{\mathrm{C}}_{\mathrm{q}, \mathrm{Ph}}\right), 128.6+128.53+128.48+128.45$ $+128.24+128.20$ (major and minor overlap, $\mathrm{CH}_{\mathrm{Ph}}$ ), 81.14 (major $\mathrm{C}_{\beta}$ ), 81.05 (major $\mathrm{CO}_{2} \underline{\mathrm{C}}\left(\mathrm{CH}_{3}\right)$ ), 81.0 (minor $\mathrm{CO}_{2} \underline{\mathrm{C}}\left(\mathrm{CH}_{3}\right)$ ), 80.2 (minor $\mathrm{C}_{\beta}$ ), 67.30 (minor $\mathrm{CH}_{2} \mathrm{Ph}$ ), 67.25 (major $\underline{\mathrm{CH}}_{2} \mathrm{Ph}$ ), 65.5 (major $\mathrm{C}_{\alpha}$ ), 65.4 (minor $\mathrm{C}_{\alpha}$ ), $59.2\left(\right.$ minor $\mathrm{C}_{\gamma}$ ), 58.5 (major $\mathrm{C}_{\gamma}$ ), 53.0 (minor $\mathrm{C}_{\delta}$ ), 52.2 (major $\mathrm{C}_{\delta}$ ), 28.3 (minor $\mathrm{CO}_{2} \mathrm{C}\left(\underline{\mathrm{CH}}_{3}\right)_{3}$ ), 28.1 (major $\left.\mathrm{CO}_{2} \mathrm{C}\left(\mathrm{CH}_{3}\right)_{3}\right)$ ppm; $R_{f} 0.34$ (hexane/acetone 70:30); MS (ESI) $(m / z) 356.1[\mathrm{M}+\mathrm{H}]^{+}$; HRMS (ESI) for $\mathrm{C}_{17} \mathrm{H}_{22} \mathrm{ClNNaO}_{5}[\mathrm{M}$ $+\mathrm{Na}]^{+}$calcd for 378.1079, found 378.1081; IR $3406(\mathrm{w}), 1738(\mathrm{~m})$, $1702(\mathrm{~s}), 1672(\mathrm{~s}), 1392(\mathrm{~s}), 1158(\mathrm{~s}), 966(\mathrm{~m}) \mathrm{cm}^{-1}$.

( \pm )-N-(tert-Butyloxycarbonyl)-(4R)-2,3-dehydro-4-hydroxyproline Benzyl Ester (34a) (Table 1, Entry 8). To a solution of epoxide 10a $(125.6 \mathrm{mg}, 0.393 \mathrm{mmol})$ in toluene $(10.0 \mathrm{~mL})$ was added $\mathrm{Bu}_{4} \mathrm{NH}_{2} \mathrm{~F}_{3}$ (tech. $90 \%, 0.1 \mathrm{~mL}$ ), and the mixture was stirred at 110 ${ }^{\circ} \mathrm{C}$. After $24 \mathrm{~h}$, the mixture was quenched with a saturated aqueous solution of $\mathrm{NaHCO}_{3}(3 \mathrm{~mL})$ and water $(10 \mathrm{~mL})$. The aqueous layer was extracted with EtOAc $(3 \times 15 \mathrm{~mL})$, and the combined organic phases were dried over $\mathrm{MgSO}_{4}$ and evaporated in vacuo. Purification by flash chromatography (hexane/EtOAc 70:30) yielded 34a as a clear oil (70.5 mg, 56\%): ${ }^{1} \mathrm{H} \mathrm{NMR}\left(400 \mathrm{MHz}, \mathrm{CDCl}_{3}\right) \delta 7.42-7.32$ $(\mathrm{m}, 5 \mathrm{H}, \mathrm{CH} \mathrm{Ar}), 5.72\left(\mathrm{~d}, J=2.8 \mathrm{~Hz}, 1 \mathrm{H}, \mathrm{C}_{\beta} \mathrm{H}\right), 5.27\left(\mathrm{~s}, 2 \mathrm{H}, \mathrm{C}_{2} \mathrm{Ph}\right)$, $4.84\left(\mathrm{tt}, J=8.0,2.8 \mathrm{~Hz}, 1 \mathrm{H}, \mathrm{C}_{\gamma} \mathrm{H}\right), 3.95(\mathrm{dd}, J=12.8,8.3 \mathrm{~Hz}, 1 \mathrm{H}$, $\left.\mathrm{C}_{\delta} \underline{\mathrm{H}} \mathrm{H}^{\prime}\right), 3.84\left(\mathrm{dd}, J=13.3,2.7 \mathrm{~Hz}, 1 \mathrm{H}, \mathrm{C}_{\delta} \mathrm{H}^{\prime}\right), 1.78(\mathrm{~d}, J=8.2 \mathrm{~Hz}$, $1 \mathrm{H}, \mathrm{OH}), 1.46\left(\mathrm{~s}, 9 \mathrm{H}, \mathrm{CO}_{2} \mathrm{C}\left(\mathrm{C}_{3}\right)_{3}\right) \mathrm{ppm} ;{ }^{13} \mathrm{C}\left\{{ }^{1} \mathrm{H}\right\}$ NMR (100 $\left.\mathrm{MHz}, \mathrm{CDCl}_{3}\right) \delta 162.1\left(\mathrm{C}_{\alpha}-\mathrm{CO}_{2}\right), 151.7\left(\mathrm{CO}_{2} \mathrm{C}\left(\mathrm{CH}_{3}\right)_{3}\right), 139.4\left(\mathrm{C}_{\alpha}\right)$, $135.1\left(\underline{\mathrm{C}}_{\mathrm{q}, \mathrm{Ph}}\right), 128.6\left(2 \mathrm{C}, \mathrm{CH}_{\mathrm{Ar}}\right), 128.5\left(1 \mathrm{C}, \mathrm{CH}_{\mathrm{Ar}}\right), 128.4(2 \mathrm{C}$, $\left.\mathrm{CH}_{\mathrm{Ar}}\right), 116.2\left(\mathrm{C}_{\beta}\right), 81.9\left(\mathrm{CO}_{2} \underline{\mathrm{C}}\left(\mathrm{CH}_{3}\right)_{3}\right), 71.0\left(\mathrm{C}_{\gamma}\right), 67.4\left(\underline{\mathrm{CH}}_{2} \mathrm{Ph}\right)$, $56.7\left(\mathrm{C}_{\delta}\right), 28.1\left(3 \mathrm{C}, \mathrm{CO}_{2} \mathrm{C}\left(\mathrm{CH}_{3}\right)_{3}\right)$ ppm; $R_{f} 0.34$ (hexane/acetone 70:30); MS (ESI) $(\mathrm{m} / z) 320.4[\mathrm{M}+\mathrm{H}]^{+}, 342.4[\mathrm{M}+\mathrm{Na}]^{+}$; HRMS (ESI) for $\mathrm{C}_{17} \mathrm{H}_{22} \mathrm{NO}_{5}[\mathrm{M}+\mathrm{H}]^{+}$calcd for 320.1492 , found 320.1495 ; IR 3434 (br w), 2978 (m), 1740 (s), 1707 (s), 1392 (s), 1368 (s), $1167(\mathrm{~s}) \mathrm{cm}^{-1}$.

( \pm )-N-(Acetyl)-(4R)-2,3-dehydro-4-hydroxyproline Benzyl Ester (34b) (Table 1, Entry 9). To a solution of epoxide $( \pm)-10 \mathrm{~b}$ $(553.0 \mathrm{mg}, 2.117 \mathrm{mmol})$ in $t-\mathrm{BuOH}(25.0 \mathrm{~mL})$ was added TBAF. $3 \mathrm{H}_{2} \mathrm{O}(1.67 \mathrm{~g}, 5.29 \mathrm{mmol})$, and the mixture was stirred at $70{ }^{\circ} \mathrm{C}$. After $4 \mathrm{~h}$, the mixture was diluted with water $(100 \mathrm{~mL})$ and the aqueous layer was extracted with DCM $(3 \times 80 \mathrm{~mL})$. The combined organic phases were dried over $\mathrm{MgSO}_{4}$ and evaporated in vacuo. Purification by flash chromatography using a Biotage purification system (hexane/acetone gradient) yielded $34 \mathrm{~b}(165.7 \mathrm{mg}, 30 \%)$ and 35 (53.2 $\mathrm{mg}, 13 \%)$ as clear oils.

Data for ( \pm )- $N$-(acetyl)-(4R)-2,3-dehydro-4-hydroxyproline Benzyl Ester (34b): ${ }^{1} \mathrm{H}$ NMR $\left(400 \mathrm{MHz}, \mathrm{CDCl}_{3}\right) \delta 7.42-7.32(\mathrm{~m}$, $\left.5 \mathrm{H}, \mathrm{CH}_{\mathrm{Ar}}\right), 5.88\left(\mathrm{~d}, J=2.9 \mathrm{~Hz}, 1 \mathrm{H}, \mathrm{C}_{\beta} \mathrm{H}\right), 5.32(\mathrm{~d}, J=12.6 \mathrm{~Hz}, 1 \mathrm{H}$, $\left.\mathrm{C}^{\mathrm{H}} \mathrm{H}^{\prime} \mathrm{Ph}\right), 5.19\left(\mathrm{~d}, J=12.6 \mathrm{~Hz}, 1 \mathrm{H}, \mathrm{CH}^{\prime} \mathrm{Ph}\right), 4.84\left(\mathrm{br} \mathrm{s}, 1 \mathrm{H}, \mathrm{C}_{\gamma} \mathrm{H}\right)$, $3.95\left(\mathrm{dd}, J=12.1,8.0 \mathrm{~Hz}, 1 \mathrm{H}, \mathrm{C}_{\delta} \underline{\mathrm{HH}^{\prime}}\right), 3.84(\mathrm{br} \mathrm{dd}, J=12.1,1.3 \mathrm{~Hz}$, $\left.1 \mathrm{H}, \mathrm{C}_{\delta} \mathrm{H}^{\prime}\right), 3.72(\mathrm{br} \mathrm{s}, 1 \mathrm{H}, \mathrm{OH}), 2.08\left(\mathrm{~s}, 3 \mathrm{H}, \mathrm{N}-\mathrm{COCH}_{3}\right) \mathrm{ppm}$; ${ }^{13} \mathrm{C}\left\{{ }^{1} \mathrm{H}\right\} \mathrm{NMR}\left(100 \mathrm{MHz}, \mathrm{CDCl}_{3}\right) \delta 168.9\left(\mathrm{~N}-\mathrm{COCH}_{3}\right), 162.2\left(\mathrm{C}_{\alpha^{-}}\right.$ $\left.\mathrm{CO}_{2}\right), 138.2\left(\mathrm{C}_{\alpha}\right), 135.1\left(\underline{\mathrm{C}}_{\mathrm{q}, \mathrm{Ph}}\right), 128.64\left(2 \mathrm{C}, \mathrm{CH}_{\mathrm{Ar}}\right), 128.59(2 \mathrm{C}$, $\left.{ }_{\mathrm{C}} \mathrm{H}_{\mathrm{Ar}}\right), 128.48\left(1 \mathrm{C}, \mathrm{CH}_{\mathrm{Ar}}\right), 119.3\left(\mathrm{C}_{\beta}\right), 71.2\left(\mathrm{C}_{\gamma}\right), 67.6\left(\mathrm{CH}_{2} \mathrm{Ph}\right)$, $56.9\left(\mathrm{C}_{\delta}\right), 22.5\left(\mathrm{~N}-\mathrm{COCH}_{3}\right) \mathrm{ppm} ; R_{f} 0.22$ (hexane/acetone 60:40); MS (ESI) $(m / z) 262.2[\mathrm{M}+\mathrm{H}]^{+}, 284.2[\mathrm{M}+\mathrm{Na}]^{+}$; HRMS (ESI) for $\mathrm{C}_{14} \mathrm{H}_{16} \mathrm{NO}_{4}[\mathrm{M}+\mathrm{H}]^{+}$calcd for 262.1074, found 262.1076; IR 3373 (br m), 2950 (m), 1733 (s), 1651 (s), 1404 (s), 1172 (s), 749 (m) $\mathrm{cm}^{-1}$.

2-Hydroxyethyl 1H-Pyrrole-2-carboxylate (36) (Table 1, Entry 10). Epoxide 10a (120.0 mg, $0.376 \mathrm{mmol})$ and $\mathrm{KHF}_{2}(146.7$ 
$\mathrm{mg}, 1.879 \mathrm{mmol})$ were dissolved in glycol $(2.0 \mathrm{~mL})$ and stirred at 120 ${ }^{\circ} \mathrm{C}$. After $4 \mathrm{~h}$, no reaction was observed and the temperature was increased to $150{ }^{\circ} \mathrm{C}$. After $21 \mathrm{~h}$, the reaction was quenched with a $5 \%$ aqueous solution of $\mathrm{K}_{2} \mathrm{CO}_{3}(4 \mathrm{~mL})$ and diluted with water $(50 \mathrm{~mL})$. Next, the aqueous layer was extracted with DCM $(5 \times 75 \mathrm{~mL})$, and the combined organic phases were dried over $\mathrm{MgSO}_{4}$ and evaporated in vacuo. Purification by flash chromatography (hexane/acetone $67: 33$ ) yielded 36 as a yellowish oil (34.4 $\mathrm{mg}, 59 \%)$, next to $1.4 \mathrm{mg}$ (2\%) of 35.

Data for 2-Hydroxyethyl Pyrrole-2-carboxylate (36): ${ }^{1} \mathrm{H}$ NMR $\left(500 \mathrm{MHz}, \mathrm{CDCl}_{3}\right) \delta 9.56($ br s, $1 \mathrm{H}, \mathrm{N} \underline{\mathrm{H}}), 6.99-6.95(\mathrm{~m}, 1 \mathrm{H}$ $\left.\mathrm{C}_{\delta} \mathrm{H}+1 \mathrm{H} \mathrm{C}_{\beta} \mathrm{H}\right), 6.27\left(\mathrm{dt}, J=3.6,2.6 \mathrm{~Hz}, 1 \mathrm{H}, \mathrm{C}_{\gamma} \mathrm{H}\right), 4.40(\mathrm{t}, J=1.7$ $\left.\mathrm{Hz}, 1 \mathrm{H}, \mathrm{CO}_{2}-\mathrm{C}_{\mathrm{H}} \mathrm{H}^{\prime}\right), 4.40$ (br d, $\left.J=9.3 \mathrm{~Hz}, 1 \mathrm{H}, \mathrm{CO}_{2}-\mathrm{CH}^{\prime}\right), 3.92$ (t, $\left.J=1.7 \mathrm{~Hz}, 1 \mathrm{H}, \mathrm{CH}^{\prime}-\mathrm{OH}\right), 3.92\left(\right.$ br d, $J=9.3 \mathrm{~Hz}, 1 \mathrm{H}, \mathrm{CH}^{\prime} \underline{\mathrm{H}}^{\prime}-$ $\mathrm{OH}), 2.64$ (br s, $1 \mathrm{H}, \mathrm{OH}) \mathrm{ppm} ;{ }^{13} \mathrm{C}\left\{{ }^{1} \mathrm{H}\right\} \mathrm{NMR}\left(126 \mathrm{MHz}, \mathrm{CDCl}_{3}\right) \delta$ $161.4\left(\mathrm{C}_{\alpha}-\underline{\mathrm{CO}_{2}}\right), 123.4\left(\mathrm{C}_{\beta}\right), 122.2\left(\mathrm{C}_{\alpha}\right), 115.9\left(\mathrm{C}_{\delta}\right), 110.6\left(\mathrm{C}_{\gamma}\right), 66.0$ $\left(\mathrm{CO}_{2}-\mathrm{CH}_{2}\right), 66.0\left(\mathrm{CH}_{2}-\mathrm{OH}\right) \mathrm{ppm} ; \mathrm{R}_{f} 0.28$ (hexane/acetone 70:30); MS (ESI) $(\mathrm{m} / z) 156.1[\mathrm{M}+\mathrm{H}]^{+}$; HRMS (ESI) for $\mathrm{C}_{7} \mathrm{H}_{9} \mathrm{NNaO}_{3}[\mathrm{M}+\mathrm{Na}]^{+}$calcd for 178.0475, found 178.0474; IR 3314 (br m), 2360 (m), 2340 (m), 1676 (s), 1408 (s), 1306 (s), 1160 (s), $742(\mathrm{~s}) \mathrm{cm}^{-1}$.

( \pm )-N-(Acetyl)-(2S,3R,4R)-3-hydroxy-4-chloroproline Benzyl Ester (32b) (Scheme 5). Epoxide ( \pm )-10b (2.55 g, $9.74 \mathrm{mmol})$ was dissolved in a $4 \mathrm{M}$ solution of $\mathrm{HCl}$ in dioxane $(50 \mathrm{~mL})$, and the mixture was stirred at room temperature for $1.5 \mathrm{~h}$. Next, the reaction mixture was cooled to $0{ }^{\circ} \mathrm{C}$ and quenched with a saturated aqueous solution of $\mathrm{NaHCO}_{3}(40 \mathrm{~mL})$. The aqueous layer was extracted with DCM $(3 \times 50 \mathrm{~mL})$, and the combined organic layers were subsequently washed with brine $(40 \mathrm{~mL})$, dried over $\mathrm{MgSO}_{4}$, and evaporated in vacuo. The crude product was purified by flash chromatography using a Biotage purification system (hexane/acetone gradient) to yield $32 \mathrm{~b}(2.77 \mathrm{~g}, 95 \%)$ as a colorless oil: ${ }^{1} \mathrm{H}$ NMR (400 $\left.\mathrm{MHz} \mathrm{CDCl}_{3}\right)$ (70:30 rotamer ratio) $\delta 7.41-7.29(\mathrm{~m}, 5 \mathrm{H}$ major $\mathrm{Ph}+$ $5 \mathrm{H}$ minor $\mathrm{Ph}$ ), $5.28\left(\mathrm{~d}, J=12.1 \mathrm{~Hz}, 1 \mathrm{H}\right.$, minor $\left.\mathrm{C}^{\mathrm{H}} \mathrm{H}^{\prime} \mathrm{Ph}\right), 5.22(\mathrm{~d}, J$ $=12.3 \mathrm{~Hz}, 1 \mathrm{H}$, major $\left.\mathrm{CHH}^{\prime} \mathrm{Ph}\right), 5.17(\mathrm{~d}, J=12.1 \mathrm{~Hz}, 1 \mathrm{H}$, minor $\mathrm{CH} \underline{\mathrm{H}}^{\prime} \mathrm{Ph}$ ), 5.14 (d, $J=12.3 \mathrm{~Hz}, 1 \mathrm{H}$, major $\mathrm{CH}^{\prime} \mathrm{Ph}$ ), 4.74 (br m, $1 \mathrm{H}$, minor $\left.\mathrm{C}_{\beta} \underline{\mathrm{H}}\right), 4.57\left(\right.$ br d, $J=2.6 \mathrm{~Hz}, 1 \mathrm{H}$, major $\left.\mathrm{C}_{\alpha} \mathrm{H}\right), 4.53(\mathrm{~m}$, $1 \mathrm{H}$, major $\left.\mathrm{C}_{\beta} \underline{\mathrm{H}}\right), 4.40\left(\mathrm{br} \mathrm{d}, J=1.2 \mathrm{~Hz}, 1 \mathrm{H}\right.$, minor $\left.\mathrm{C}_{\alpha} \underline{\mathrm{H}}\right), 4.27-4.04$ (m, $1 \mathrm{H}$ major $\mathrm{C}_{\gamma} \underline{\mathrm{H}}+1 \mathrm{H}$ minor $\mathrm{C}_{\gamma} \underline{\mathrm{H}}+1 \mathrm{H}$ major $\mathrm{C}_{\delta} \underline{\mathrm{HH}^{\prime}}+1 \mathrm{H}$ minor $\mathrm{C}_{\delta} \mathrm{HH}^{\prime}+1 \mathrm{H}$ major $\mathrm{OH}+1 \mathrm{H}$ minor $\left.\mathrm{OH}\right), 3.81-3.74(\mathrm{~m}, 1 \mathrm{H}$ major $\mathrm{C}_{\delta} \mathrm{H}^{\prime}+1 \mathrm{H}$ minor $\left.\mathrm{C}_{\delta} \mathrm{HH}^{\prime}\right), 2.08\left(\mathrm{~s}, 3 \mathrm{H}\right.$, major $\left.\mathrm{CH}_{3}\right), 1.96(\mathrm{~s}, 3 \mathrm{H}$, minor $\left.\mathrm{CH}_{3}\right)$ ppm; ${ }^{13} \mathrm{C}\left\{{ }^{1} \mathrm{H}\right\}$ NMR $\left(100 \mathrm{MHz}, \mathrm{CDCl}_{3}\right)(70: 30$ rotamer ratio) $\delta 171.1$ (minor $\left.\mathrm{N}-\mathrm{COCH}_{3}\right), 170.4$ (major $\left.\mathrm{N}-\mathrm{COCH}_{3}\right), 168.6$ (1C major $\mathrm{C}_{\alpha}-\underline{\mathrm{CO}}_{2}+1 \mathrm{C}$ minor $\mathrm{C}_{\alpha}-\underline{\mathrm{CO}}_{2}$ ), 135.3 (major $\underline{\mathrm{C}}_{\mathrm{q}, \mathrm{Ph}}$ ), 134.8 $\left(\right.$ minor $\left.\underline{\mathrm{C}}_{\mathrm{q}, \mathrm{Ph}}\right), 128.74+128.70+128.68+128.6+128.52+128.46+$ $128.4+128.3$ (major and minor overlap, $\underline{\mathrm{CH}}_{\mathrm{Ph}}$ ), $81.3\left(\right.$ minor $\left.\underline{\mathrm{C}}_{\beta} \mathrm{H}\right)$, 79.5 (major $\left.\underline{\mathrm{C}}_{\beta} \mathrm{H}\right), 67.9$ (minor $\left.\underline{\mathrm{CH}}_{2} \mathrm{Ph}\right), 67.4$ (major $\left.\underline{\mathrm{CH}}_{2} \mathrm{Ph}\right), 67.0$ (minor $\underline{\mathrm{C}}_{\alpha} \mathrm{H}$ ), 65.3 (major $\underline{\mathrm{C}}_{\alpha} \mathrm{H}$ ), 59.2 (major $\underline{\mathrm{C}}_{\gamma} \mathrm{H}$ ), 58.3 (minor $\underline{\mathrm{C}}_{\gamma} \mathrm{H}$ ), 54.2 (major $\underline{\mathrm{C}}_{\delta} \mathrm{H}_{2}$ ), 53.4 (minor $\underline{\mathrm{C}}_{\delta} \mathrm{H}_{2}$ ), 22.0 (minor $\underline{\mathrm{CH}}_{3}$ ), 21.9 (major $\mathrm{CH}_{3}$ ) ppm; $R_{f} 0.25$ (hexane/acetone 60:40); MS (ESI) $(m / z) 298.3[\mathrm{M}+\mathrm{H}]^{+}, 320.3[\mathrm{M}+\mathrm{Na}]^{+}$; HRMS (ESI) for $\mathrm{C}_{14} \mathrm{H}_{17}$ $\mathrm{ClNO}_{4}[\mathrm{M}+\mathrm{H}]^{+}$calcd for 298.0841, found 298.0834; IR 3304 (br m), $1747(\mathrm{~s}), 1628(\mathrm{~s}), 1189(\mathrm{~m}), 1175(\mathrm{~m}), 698(\mathrm{~m}) \mathrm{cm}^{-1}$.

(t)-N-(Acetyl)-(2S,3R,4R)-3-hydroxy-4-(4-methylphenyl)sulfonyl Proline Benzyl Ester (37b) (Scheme 5). $p$-Toluenesulfonic acid monohydrate $(640.6 \mathrm{mg}, 3.368 \mathrm{mmol})$ was added to a solution of epoxide $( \pm)-10 \mathrm{~b}(220.0 \mathrm{mg}, 0.842 \mathrm{mmol})$ in DCM $(6.0$ $\mathrm{mL}$ ) and the mixture stirred at $45{ }^{\circ} \mathrm{C}$ for $6 \mathrm{~h}$. Next, the mixture was diluted with DCM $(10 \mathrm{~mL})$, and the organic phase was washed with a saturated aqueous solution of $\mathrm{NaHCO}_{3}(2 \times 7 \mathrm{~mL})$ and brine $(1 \times 10$ $\mathrm{mL})$. The organic phase was then dried over $\mathrm{MgSO}_{4}$ and evaporated in vacuo. Purification via HPLC (hexane/acetone 60:40) yielded $37 \mathbf{b}$ as a clear oil $(265.0 \mathrm{mg}, 73 \%):{ }^{1} \mathrm{H}$ NMR $\left(400 \mathrm{MHz}, \mathrm{CDCl}_{3}\right)(66: 34$ rotamer ratio) $\delta 7.75-7.65(\mathrm{~m}, 2 \mathrm{H}$ major $\mathrm{CH} \mathrm{Ar}+2 \mathrm{H}$ minor $\mathrm{CH}$ $\mathrm{Ar}), 7.41-7.28(\mathrm{~m}, 7 \mathrm{H}$ major $\mathrm{CH} \mathrm{Ar}+7 \mathrm{H}$ minor $\mathrm{CH} \mathrm{Ar}), 5.19(\mathrm{~d}, J$ $=12.1 \mathrm{~Hz}, 1 \mathrm{H}$, minor $\left.\mathrm{CHH}^{\prime} \mathrm{Ph}\right), 5.14(\mathrm{~d}, J=12.5 \mathrm{~Hz}, 1 \mathrm{H}$, major $\left.\mathrm{CH}^{\prime} \mathrm{Ph}\right), 5.10\left(\mathrm{~d}, J=12.1 \mathrm{~Hz}, 1 \mathrm{H}\right.$, minor $\left.\mathrm{CH} \underline{H}^{\prime} \mathrm{Ph}\right), 5.03(\mathrm{~d}, J=$ $12.5 \mathrm{~Hz}, 1 \mathrm{H}$, major $\left.\mathrm{CHH}^{\prime} \mathrm{Ph}\right), 4.86(\mathrm{dt}, J=5.2,2.5 \mathrm{~Hz}, 1 \mathrm{H}$, major $\left.\mathrm{C}_{\gamma} \mathrm{H}\right)$, 4.81-4.76 $\left(\mathrm{m}, 1 \mathrm{H}\right.$ minor $\mathrm{C}_{\gamma} \mathrm{H}+1 \mathrm{H}$ minor $\left.\mathrm{C}_{\beta} \mathrm{H}\right), 4.58(\mathrm{~d}, J=$ $1.8 \mathrm{~Hz}, 1 \mathrm{H}$ major $\left.\mathrm{C}_{\alpha} \mathrm{H}\right), 4.50$ (br s, $1 \mathrm{H}$ major $\mathrm{C}_{\beta} \mathrm{H}$ ), $4.38($ br s, $1 \mathrm{H}$ minor $\mathrm{C}_{\alpha} \mathrm{H}$ ), 4.06 (br s, $1 \mathrm{H}$ minor $\mathrm{OH}$ ), 3.95-3.83 (m, $1 \mathrm{H}$ major $\mathrm{OH}+1 \mathrm{H}$ major $\mathrm{C}_{\delta} \underline{\mathrm{H}} \mathrm{H}^{\prime}+1 \mathrm{H}$ minor $\left.\mathrm{C}_{\delta} \underline{\mathrm{H}}^{\prime}\right), 3.67(\mathrm{~d}, J=11.9,2.4$ $\mathrm{Hz}, 1 \mathrm{H}$ major $\left.\mathrm{C}_{\delta} \mathrm{H}^{\prime}\right)$, $3.53\left(\mathrm{br} \mathrm{d}, J=14.1 \mathrm{~Hz}, 1 \mathrm{H}\right.$ minor $\left.\mathrm{C}_{\delta} \mathrm{H}^{\prime}\right)$, $2.44\left(\mathrm{~s}, 3 \mathrm{H}\right.$, major OTs $\left.\mathrm{CH}_{3}\right), 2.43\left(\mathrm{~s}, 3 \mathrm{H}\right.$, minor $\left.\mathrm{OTs}_{\mathrm{CH}}\right), 2.00(\mathrm{~s}$, $3 \mathrm{H}$, major $\left.\mathrm{N}-\mathrm{COCH}_{3}\right), 1.91\left(\mathrm{~s}, 3 \mathrm{H}\right.$, minor $\left.\mathrm{N}-\mathrm{COCH}_{3}\right) \mathrm{ppm}$; ${ }^{13} \mathrm{C}\left\{{ }^{1} \mathrm{H}\right\}$ NMR (100 MHz, $\left.\mathrm{CDCl}_{3}\right)(66: 34$ rotamer ratio) $\delta 170.8$ $\left(\right.$ minor $\left.\mathrm{N}-\underline{\mathrm{COCH}}{ }_{3}\right), 170.4$ (major $\left.\mathrm{N}-\underline{\mathrm{COCH}}{ }_{3}\right), 168.2$ (minor $\mathrm{C}_{\alpha^{-}}$ $\mathrm{CO}_{2}$ ), 168.1 (major $\mathrm{C}_{\alpha}-\mathrm{CO}_{2}$ ), 145.53 (major OTs $\underline{\mathrm{C}}_{\mathrm{q}(\mathrm{Me})}$ ), 145.50 (minor OTs $\underline{\mathrm{C}}_{\mathrm{q}(\mathrm{Me})}$ ), 135.3 (major $\left.\mathrm{C}_{\mathrm{q}(\mathrm{Bn})}\right), 134.8$ (minor $\mathrm{C}_{\mathrm{q}(\mathrm{Bn})}$ ), 133.0 (minor OTs $\underline{C}_{\mathrm{q}(\mathrm{SO} 2)}$ ), 132.9 (major OTs $\mathrm{C}_{\mathrm{q}(\mathrm{SO} 2)}$ ), 130.09 (minor $\underline{\mathrm{C}} \mathrm{H}_{\mathrm{Ar}}$ ), 130.05 (major $\left.\underline{\mathrm{CH}}_{\mathrm{Ar}}\right), 128.74+128.67+128.6+$ $128.5+128.3+128.2+128.0+127.9+127.7$ (major and minor overlap, $\mathrm{CH}_{\mathrm{Ar}}$ ), 81.8 (major $\mathrm{C}_{\gamma}$ ), 80.9 (minor $\mathrm{C}_{\gamma}$ ), 78.3 (minor $\mathrm{C}_{\beta}$ ), 76.2 (major $\mathrm{C}_{\beta}$ ), 68.0 (minor $\left.\underline{\mathrm{C}}_{2} \mathrm{Ph}\right), 67.4$ (major $\left.\underline{\mathrm{C}}_{2} \mathrm{Ph}\right), 66.8$ (minor $\left.\mathrm{C}_{\alpha}\right), 65.1\left(\right.$ major $\left.\mathrm{C}_{\alpha}\right), 51.1\left(\right.$ major $\left.\mathrm{C}_{\delta}\right), 49.9\left(\right.$ minor $\left.\mathrm{C}_{\delta}\right), 21.9$ (2C, minor $\mathrm{N}-\mathrm{CO}_{\mathrm{CH}} \mathrm{H}_{3}+$ major $\left.\mathrm{N}-\mathrm{CO} \underline{\mathrm{CH}}_{3}\right), 21.7$ (2C, minor OTs $\mathrm{CH}_{3}+$ major OTs $\mathrm{CH}_{3}$ ) ppm; $R_{f} 0.34$ (hexane/acetone 60:40); MS (ESI) $(m / z) 434.3[\mathrm{M}+\mathrm{H}]^{+}, 456.1[\mathrm{M}+\mathrm{Na}]^{+}$; HRMS (ESI) for $\mathrm{C}_{21} \mathrm{H}_{24} \mathrm{NO}_{7} \mathrm{~S}[\mathrm{M}+\mathrm{H}]^{+}$calcd for 434.1268, found 434.1271; IR 3322 (br w), 1749 (m), 1627 (m), 1189 (s), 1175 (s), $732(\mathrm{~s}) \mathrm{cm}^{-1}$.

( \pm -) $N$-(Acetyl)-(2S,R,4R)-3-hydroxy-4-bromoproline Benzyl Ester (38b) (Scheme 5). Reaction with $\mathrm{MgBr}_{2}$. To a solution of epoxide $( \pm)-10 \mathrm{~b}(147.7 \mathrm{mg}, 0.565 \mathrm{mmol})$ in DCM $(4.0 \mathrm{~mL})$ was added $\mathrm{MgBr}_{2}$ (156.1 mg, $0.848 \mathrm{mmol}$ ), and the mixture was stirred at room temperature. After $20 \mathrm{~h}$, the mixture was diluted with DCM (10 $\mathrm{mL})$, water $(10 \mathrm{~mL})$, and a saturated aqueous solution of $\mathrm{NaHCO}_{3}(5$ $\mathrm{mL})$. The aqueous layer was extracted with DCM $(3 \times 10 \mathrm{~mL})$, and the combined organic phases were dried over $\mathrm{MgSO}_{4}$ and evaporated in vacuo. Bromohydrin $\mathbf{3 8 b}$ was obtained without purification as a clear oil (169.9 mg, 88\%).

Reaction with $\mathrm{HBr} . \mathrm{HBr}\left(48\right.$ wt $\%$ in $\mathrm{H}_{2} \mathrm{O}, 1.0 \mathrm{~mL}$ ) was added dropwise to a solution of epoxide $( \pm)-10 \mathrm{~b}(142.1 \mathrm{mg}, 0.543 \mathrm{mmol})$ in DCM $(3.0 \mathrm{~mL})$ at $0{ }^{\circ} \mathrm{C}$. After $2 \mathrm{~h}$, the mixture was quenched with a saturated aqueous solution of $\mathrm{NaHCO}_{3}(5 \mathrm{~mL})$, and the aqueous layer was extracted with $\mathrm{DCM}(3 \times 7 \mathrm{~mL})$. The combined organic phases were washed with brine $(1 \times 10 \mathrm{~mL})$, dried over $\mathrm{MgSO}_{4}$, and evaporated in vacuo. Bromohydrin $\mathbf{3 8 b}$ was obtained without purification as a clear oil (177.5 mg, 95\%): ${ }^{1} \mathrm{H}$ NMR $(400 \mathrm{MHz}$, $\left.\mathrm{CDCl}_{3}\right)(71: 29$ rotamer ratio) $\delta 7.42-7.30(\mathrm{~m}, 5 \mathrm{H}$ major $\mathrm{Ph}+5 \mathrm{H}$ minor $\mathrm{Ph}), 5.29\left(\mathrm{~d}, J=12.0 \mathrm{~Hz}, 1 \mathrm{H}\right.$, minor $\left.\mathrm{CH}^{\prime} \mathrm{Ph}\right), 5.24(\mathrm{~d}, J=$ $12.4 \mathrm{~Hz}, 1 \mathrm{H}$, major $\left.\mathrm{CHH}^{\prime} \mathrm{Ph}\right), 5.19(\mathrm{~d}, J=12.0 \mathrm{~Hz}, 1 \mathrm{H}$, minor $\mathrm{CH} \underline{H}^{\prime} \mathrm{Ph}$ ), $5.16\left(\mathrm{~d}, J=12.4 \mathrm{~Hz}, 1 \mathrm{H}\right.$, major $\mathrm{CH} \underline{H}^{\prime} \mathrm{Ph}$ ), 4.82 (br app. t, $1 \mathrm{H}$, minor $\left.\mathrm{C}_{\beta} \mathrm{H}\right), 4.59$ (app. t, $J=3.7 \mathrm{~Hz}, 1 \mathrm{H}$, major $\left.\mathrm{C}_{\beta} \mathrm{H}\right), 4.51(\mathrm{~d}, J$ $=3.6 \mathrm{~Hz}, 1 \mathrm{H}$, major $\left.\mathrm{C}_{\alpha} \mathrm{H}\right), 4.51\left(\mathrm{~d}, J=2.0 \mathrm{~Hz}, 1 \mathrm{H}\right.$, minor $\left.\mathrm{C}_{\alpha} \mathrm{H}\right)$, 4.38-4.11 (m, $1 \mathrm{H}$ major $\mathrm{C}_{\gamma} \mathrm{H}+1 \mathrm{H}$ minor $\mathrm{C}_{\gamma} \mathrm{H}+1 \mathrm{H}$ major $\mathrm{C}_{\delta} \underline{\mathrm{H}} \mathrm{H}^{\prime}+$ $1 \mathrm{H}$ minor $\left.\mathrm{C}_{\delta} \underline{\mathrm{HH}^{\prime}}\right), 3.92-3.80\left(\mathrm{~m}, 1 \mathrm{H}\right.$ major $\mathrm{C}_{\delta} \mathrm{HH}^{\prime}+1 \mathrm{H}$ major $\mathrm{C}_{\delta} \mathrm{H}^{\prime}$ ), 3.74-3.57 (br s, $1 \mathrm{H}$ major $\mathrm{OH}+1 \mathrm{H}$ minor $\mathrm{OH}$ ), 2.09 (s, $3 \mathrm{H}$, major $\left.\mathrm{CH}_{3}\right), 1.96\left(\mathrm{~s}, 3 \mathrm{H}\right.$, minor $\left.\mathrm{CH}_{3}\right) \mathrm{ppm} ;{ }^{13} \mathrm{C}\left\{{ }^{1} \mathrm{H}\right\}$ NMR $(100$ $\mathrm{MHz}, \mathrm{CDCl}_{3}$ ) (71:29 rotamer ratio) $\delta 170.8$ (minor $\mathrm{N}-\mathrm{COCH}_{3}$ ), 169.9 (major $\mathrm{N}-\mathrm{COCH}_{3}$ ), 168.9 (major $\mathrm{C}_{\alpha^{-}}-\mathrm{CO}_{2}$ ), 168.8 (minor $\mathrm{C}_{\alpha^{-}}$ $\mathrm{CO}_{2}$ ), 135.3 (major $\underline{\mathrm{C}}_{\mathrm{q}, \mathrm{Ph}}$ ), 134.7 (minor $\underline{\mathrm{C}}_{\mathrm{q}, \mathrm{Ph}}$ ), $128.8+128.72+$ $128.70+128.6+128.4+128.3$ (major and minor overlap, $\underline{\mathrm{CH}}_{\mathrm{Ph}}$ ), 81.8 (minor $\mathrm{C}_{\beta}$ ), 80.0 (major $\mathrm{C}_{\beta}$ ), 68.0 (minor $\mathrm{CH}_{2} \mathrm{Ph}$ ), 67.4 (major $\mathrm{CH}_{2} \mathrm{Ph}$ ), 66.9 (minor $\mathrm{C}_{\alpha}$ ), 65.0 (major $\mathrm{C}_{\alpha}$ ), $54.3\left(\right.$ major $\mathrm{C}_{\delta}$ ), 53.6 (minor $\mathrm{C}_{\delta}$ ), 47.7 (major $\mathrm{C}_{\gamma}$ ), 47.2 (minor $\mathrm{C}_{\gamma}$ ), 21.9 (minor $\mathrm{N}$ $\mathrm{COCH}_{3}$ ), 21.8 (major N-COC $\left.\mathrm{CH}_{3}\right)$ ppm; $R_{f} 0.32$ (hexane/acetone 60:40); MS (ESI) $(\mathrm{m} / z) 342.1[\mathrm{M}+\mathrm{H}]^{+}, 364.1[\mathrm{M}+\mathrm{Na}]^{+}$; HRMS (ESI) for $\mathrm{C}_{14} \mathrm{H}_{17} \mathrm{BrNO}_{4}[\mathrm{M}+\mathrm{H}]^{+}$calcd for 342.0335, found 342.0332; IR 3296 (br w), 1742 (s), 1625 (s), 1171 (s), 733 (s), 697 (m) $\mathrm{cm}^{-1}$.

Benzyl N-Acetyl pyrrole-2-carboxylate (39b) (Scheme 5). At $-78{ }^{\circ} \mathrm{C}$, DAST $(60.0 \mu \mathrm{L}, 0.420 \mathrm{mmol})$ was added to a solution of chlorohydrin 32b $(83.4 \mathrm{mg}, 0.280 \mathrm{mmol})$ in DCM $(1.0 \mathrm{~mL})$. The reaction was allowed to warm to room temperature, and after $22 \mathrm{~h}$, TLC analysis indicated full conversion of the starting material. Next, the reaction was diluted with DCM $(4 \mathrm{~mL})$ and quenched with a saturated aqueous solution of $\mathrm{NaHCO}_{3}(2 \mathrm{~mL})$ and water $(2 \mathrm{~mL})$. The aqueous layer was extracted with DCM $(3 \times 8 \mathrm{~mL})$, and the combined organic phases were dried over $\mathrm{MgSO}_{4}$ and evaporated in vacuo. Purification via HPLC (hexane/acetone 80:20) yielded pyrrole 
39b as a clear oil $(27.4 \mathrm{mg}, 40 \%):{ }^{1} \mathrm{H}$ NMR $\left(400 \mathrm{MHz}, \mathrm{CDCl}_{3}\right) \delta$ 7.45-7.32 (m, 6H, $\left.1 \mathrm{H} \mathrm{C}_{\beta} \mathrm{H} / \mathrm{C}_{\delta} \mathrm{H}+5 \mathrm{H} \mathrm{Ph}\right), 7.02(\mathrm{dd}, J=3.6,1.7 \mathrm{~Hz}$, $\left.1 \mathrm{H}, \mathrm{C}_{\beta} \mathrm{H} / \mathrm{C}_{\delta} \mathrm{H}\right), 6.23\left(\mathrm{t}, J=3.4 \mathrm{~Hz}, 1 \mathrm{H}, \mathrm{C}_{\gamma} \mathrm{H}\right), 5.31\left(\mathrm{~s}, 2 \mathrm{H}, \mathrm{C}_{2} \mathrm{Ph}\right)$, $2.60\left(\mathrm{~s}, 3 \mathrm{H}, \mathrm{N}-\mathrm{COC} \mathrm{H}_{3}\right) \mathrm{ppm} ;{ }^{13} \mathrm{C}\left\{{ }^{1} \mathrm{H}\right\}$ NMR $\left(100 \mathrm{MHz}, \mathrm{CDCl}_{3}\right) \delta$ $169.2\left(\mathrm{~N}-\mathrm{COCH}_{3}\right), 160.8\left(\mathrm{C}_{\alpha}-\underline{\mathrm{CO}_{2}}\right), 135.7\left(\underline{\mathrm{C}}_{\mathrm{q}, \mathrm{Ph}}\right), 128.6(2 \mathrm{C}$, $\left.\underline{\mathrm{C}}_{\mathrm{Ph}}\right), 128.3\left(1 \mathrm{C}, \underline{\mathrm{C}}_{\mathrm{Ph}}\right), 128.2\left(2 \mathrm{C}, \underline{\mathrm{C}} \mathrm{H}_{\mathrm{Ph}}\right), 126.5,124.8\left(\underline{\mathrm{C}}_{\alpha}\right)$, 123.1, $110.9\left(\underline{\mathrm{C}}_{\gamma}\right), 66.7\left(\underline{\mathrm{CH}}_{2} \mathrm{Ph}\right), 24.9\left(\mathrm{~N}-\mathrm{COCH}_{3}\right) \mathrm{ppm} ; R_{f} 0.64$ (hexane/acetone 60:40); MS (ESI) $(\mathrm{m} / \mathrm{z}) 244.2[\mathrm{M}+\mathrm{H}]^{+}, 266.2[\mathrm{M}$ $+\mathrm{Na}]^{+}$; HRMS (ESI) for $\mathrm{C}_{14} \mathrm{H}_{13} \mathrm{NNaO}_{3}[\mathrm{M}+\mathrm{Na}]^{+}$calcd for 266.0788, found 266.0785; IR 1715 (s), 1257 (s), 1096 (s), 756 (m) $\mathrm{cm}^{-1}$.

(+)-N-(Acetyl)-(2R,3S,4R)-3-fluoro-4-(4-methylphenyl)sulfonyl Proline Benzyl Ester (40b) (Scheme 5). At $-78{ }^{\circ} \mathrm{C}$, DAST $(31.0 \mu \mathrm{L}, 0.238 \mathrm{mmol})$ was added to a solution of $( \pm)-37 \mathbf{b}$ $(102.2 \mathrm{mg}, 0.238 \mathrm{mmol})$ in DCM $(2.0 \mathrm{~mL})$. After the mixture was stirred at $-78{ }^{\circ} \mathrm{C}$ for $8 \mathrm{~h}$, another 2 equiv of DAST $(62.0 \mu \mathrm{L}, 0.476$ $\mathrm{mmol}$ ) was added. Overnight, the mixture was allowed to warm to room temperature. Next, the reaction was diluted with DCM $(10 \mathrm{~mL})$ and quenched with a saturated aqueous solution of $\mathrm{NaHCO}_{3}(5 \mathrm{~mL})$. The aqueous layer was then extracted with DCM $(3 \times 10 \mathrm{~mL})$, and the combined organic phases were dried over $\mathrm{MgSO}_{4}$ and evaporated in vacuo. Purification via HPLC (hexane/acetone 70:30) yielded pyrrole $39 \mathrm{~b}(35.8 \mathrm{mg}, 62 \%)$ and $40 \mathrm{~b}(6.3 \mathrm{mg}, 6 \%)$.

Data for ( \pm )-N-(Acetyl)-(2R,3S,4R)-3-fluoro-4-(4-methylphenyl)sulfonyl Proline Benzyl Ester (40b): ${ }^{1} \mathrm{H}$ NMR(400 $\left.\mathrm{MHz}, \mathrm{CDCl}_{3}\right)$ (83:17 rotamer ratio) $\delta 7.86-7.77(\mathrm{~m}, 2 \mathrm{H}$ major $\mathrm{CH} \mathrm{Ar}+2 \mathrm{H}$ minor $\mathrm{CH} \mathrm{Ar}), 7.43-7.29(\mathrm{~m}, 7 \mathrm{H}$ major $\mathrm{CH} \mathrm{Ar}+7 \mathrm{H}$ minor $\mathrm{CH} \mathrm{Ar}), 5.38$ $\left(\mathrm{dt}, J=52.9,4.8 \mathrm{~Hz}, 1 \mathrm{H}\right.$, minor $\left.\mathrm{C}_{\beta} \mathrm{H}\right), 5.27-5.08\left(\mathrm{~m}, 1 \mathrm{H}\right.$ major $\mathrm{C}_{\beta} \mathrm{H}$ $+2 \mathrm{H}$ major $\underline{\mathrm{C}}_{2} \mathrm{Ph}+2 \mathrm{H}$ minor $\left.\underline{\mathrm{C}}_{2} \mathrm{Ph}\right), 4.88-4.75(\mathrm{~m}, 1 \mathrm{H}$ major $\mathrm{C}_{\gamma} \mathrm{H}+1 \mathrm{H}$ minor $\left.\mathrm{C}_{\gamma} \mathrm{H}\right), 4.70\left(\mathrm{dd}, J=28.1,4.8 \mathrm{~Hz}, 1 \mathrm{H}\right.$, major $\left.\mathrm{C}_{\alpha} \mathrm{H}\right)$, $4.63\left(\mathrm{dd}, J=23.5,5.1 \mathrm{~Hz}, 1 \mathrm{H}\right.$, minor $\left.\mathrm{C}_{\alpha} \mathrm{H}\right), 4.18(\mathrm{dd}, J=11.7,7.3$ $\mathrm{Hz}, 1 \mathrm{H}$, minor $\left.\mathrm{C}_{\delta} \mathrm{H}^{\prime}\right), 3.97(\mathrm{dd}, J=10.0,7.5 \mathrm{~Hz}, 1 \mathrm{H}$, major $\left.\mathrm{C}_{\delta} \underline{\mathrm{H}} \mathrm{H}^{\prime}\right), 3.80\left(\mathrm{t}, J=10.0 \mathrm{~Hz}, 1 \mathrm{H}\right.$, major $\left.\mathrm{C}_{\delta} \mathrm{H}^{\prime}\right), 3.45(\mathrm{t}, J=10.6 \mathrm{~Hz}$, $1 \mathrm{H}$, minor $\left.\mathrm{C}_{\delta} \mathrm{HH}^{\prime}\right), 2.48\left(\mathrm{~s}, 3 \mathrm{H}\right.$, major OTs $\left.\mathrm{CH}_{3}\right), 2.47$ (s, 3H, minor OTs $\left.\mathrm{CH}_{3}\right), 2.11\left(\mathrm{~s}, 3 \mathrm{H}\right.$, major $\left.\mathrm{N}-\mathrm{COCH}_{3}\right), 1.84(\mathrm{~s}, 3 \mathrm{H}$, minor $\mathrm{N}$ $\left.\mathrm{COCH}_{3}\right)$ ppm; ${ }^{13} \mathrm{C}\left\{{ }^{1} \mathrm{H}\right\}$ NMR $\left(100 \mathrm{MHz}, \mathrm{CDCl}_{3}\right)(83: 17$ rotamer ratio) $\delta 169.7$ (minor $\left.\mathrm{N}-\mathrm{COCH}_{3}\right), 169.2\left(\right.$ major $\left.\mathrm{N}-\mathrm{COCH}_{3}\right), 168.2$ $\left(\mathrm{d}, J=7.3 \mathrm{~Hz}\right.$, minor $\left.\mathrm{C}_{\alpha}-\underline{\mathrm{C}} \mathrm{O}_{2}\right), 164.8\left(\mathrm{~d}, J=8.1 \mathrm{~Hz}\right.$, major $\left.\mathrm{C}_{\alpha}-\mathrm{CO}_{2}\right)$, 145.9 (major OTs $\underline{\mathrm{C}}_{\mathrm{q}(\mathrm{Me})}$ ), 145.8 (minor OTs $\underline{\mathrm{C}}_{\mathrm{q}(\mathrm{Me})}$ ), 135.1 (major $\left.\mathrm{C}_{\mathrm{q}(\mathrm{Bn})}\right), 134.5\left(\operatorname{minor} \mathrm{C}_{\mathrm{q}(\mathrm{Bn})}\right), 132.6$ (minor OTs $\left.\underline{\mathrm{C}}_{\mathrm{q}(\mathrm{SO} 2)}\right), 132.4$ (major OTs $\left.\underline{\mathrm{C}}_{\mathrm{q}(\mathrm{SO} 2)}\right), 130.2+128.8+128.7+128.5+128.4+128.2$ $+127.9+127.9$ (major and minor overlap, $\left.\mathrm{CH}_{\mathrm{Ar}}\right), 90.0(\mathrm{~d}, J=196.6$ $\mathrm{Hz}$, minor $\left.\mathrm{C}_{\beta}\right), 88.2\left(\mathrm{~d}, J=193.7\right.$, major $\left.\mathrm{C}_{\beta}\right), 73.8(\mathrm{~d}, J=17.6 \mathrm{~Hz}$, major $\left.\mathrm{C}_{\gamma}\right), 73.4\left(\mathrm{~d}, J=16.9 \mathrm{~Hz}\right.$, minor $\left.\mathrm{C}_{\gamma}\right), 68.2\left(\operatorname{minor} \mathrm{CH}_{2} \mathrm{Ph}\right), 67.6$ (major $\left.\mathrm{CH}_{2} \mathrm{Ph}\right), 62.5\left(\mathrm{~d}, J=22.0 \mathrm{~Hz}\right.$, minor $\left.\mathrm{C}_{\alpha}\right), 61.2(\mathrm{~d}, J=21.3 \mathrm{~Hz}$, major $\left.\mathrm{C}_{\alpha}\right), 48.0\left(\mathrm{~d}, J=1.5 \mathrm{~Hz}\right.$, major $\left.\mathrm{C}_{\delta}\right), 46.0(\mathrm{~d}, J=1.5 \mathrm{~Hz}$, minor $\mathrm{C}_{\delta}$ ), 22.0 (major $\mathrm{N}-\mathrm{COCH}_{3}$ ), 21.73 (major OTs- $\mathrm{CH}_{3}$ ), 21.71 (minor OTs- $\left.\mathrm{CH}_{3}\right), 21.2\left(\right.$ minor $\left.\mathrm{N}-\mathrm{COCH}_{3}\right)$ ppm; ${ }^{19} \mathrm{~F}$ NMR $(376 \mathrm{MHz}$, $\left.\mathrm{CDCl}_{3}\right)(82: 18$ rotamer ratio) $\delta-202.6(\mathrm{ddd}, J=52.9,23.4,19.1 \mathrm{~Hz}$, $1 \mathrm{~F}$, minor F), -204.5 (ddd, $J=53.3,27.3,21.7 \mathrm{~Hz}, 1 \mathrm{~F}$, major $\mathrm{F}$ ) ppm; ${ }^{19} \mathrm{~F}\left\{{ }^{1} \mathrm{H}\right\}$ NMR (376 $\left.\mathrm{MHz}, \mathrm{CDCl}_{3}\right)$ (82:18 rotamer ratio) $\delta$ -202.6 (s, 1F, minor F), -204.5 (s, 1F, major F) ppm; $R_{f} 0.42$ (hexane/acetone 60:40); MS (ESI) $(\mathrm{m} / \mathrm{z}) 436.3[\mathrm{M}+\mathrm{H}]^{+}, 458.3[\mathrm{M}$ $+\mathrm{Na}]^{+}$; HRMS (ESI) for $\mathrm{C}_{21} \mathrm{H}_{23} \mathrm{FNO}_{6} \mathrm{~S}[\mathrm{M}+\mathrm{H}]^{+}$calcd for 436.1225, found 436.1229; IR $1759(\mathrm{~m}), 1659(\mathrm{~m}), 1414(\mathrm{~m}), 1368$ (m), $1191(\mathrm{~s}), 1176(\mathrm{~s}) \mathrm{cm}^{-1}$.

Reaction of $37 b$ with TBAF/t-BuOH (Scheme 5). TBAF $3 \mathrm{H}_{2} \mathrm{O}$ (143.6 mg, $0.455 \mathrm{mmol}$ ) was added to a solution of $37 \mathrm{~b}(78.9 \mathrm{mg}$, $0.154 \mathrm{mmol})$ in $t-\mathrm{BuOH}(5.0 \mathrm{~mL})$, and the resulting mixture was stirred at $50{ }^{\circ} \mathrm{C}$. After $2 \mathrm{~h}$, the mixture was poured in water $(20 \mathrm{~mL})$ and extracted with DCM $(3 \times 15 \mathrm{~mL})$. The combined organic phases were dried over $\mathrm{MgSO}_{4}$ and evaporated in vacuo. Purification by flash chromatography (hexane/acetone 60:40) yielded allylic alcohol 34b $(22.7 \mathrm{mg}, 48 \%)$ and epoxide $10 \mathrm{~b}(9.0 \mathrm{mg}, 19 \%)$.

Reaction of $38 \mathrm{~b}$ with TBAF/t-BuOH (Scheme 5). TBAF- $3 \mathrm{H}_{2} \mathrm{O}$ (194.4 mg, $0.616 \mathrm{mmol}$ ) was added to a solution of $38 \mathrm{~b}(52.7 \mathrm{mg}$, $0.154 \mathrm{mmol})$ in $t-\mathrm{BuOH}(2.5 \mathrm{~mL})$, and the resulting mixture was stirred at $80^{\circ} \mathrm{C}$. After $1 \mathrm{~h}$, the mixture was poured in water $(20 \mathrm{~mL})$ and extracted with DCM $(3 \times 10 \mathrm{~mL})$. The combined organic phases were dried over $\mathrm{MgSO}_{4}$ and evaporated in vacuo. Purification by flash chromatography (hexane/acetone 60:40) yielded allylic alcohol 34b $(33.7 \mathrm{mg}, 84 \%)$ as a clear oil.

Reaction of $\mathbf{3 8 b}$ with $\mathrm{AgF}$ (Scheme 5). To a solution of $\mathbf{3 8 \mathbf { b }}$ (99.0 $\mathrm{mg}, 0.289 \mathrm{mmol})$ in nitromethane $(5.0 \mathrm{~mL})$ was added AgF $(183.5$ $\mathrm{mg}, 1.447 \mathrm{mmol}$ ), and the resulting mixture was stirred at room temperature. After $2 \mathrm{~h}$, no conversion of the starting material was observed on TLC and subsequently the mixture was heated at $45{ }^{\circ} \mathrm{C}$. After $15 \mathrm{~h}$, the mixture was filtered through Celite and the solvent evaporated in vacuo to yield $10 \mathrm{~b}(77.0 \mathrm{mg}$, quant) as a clear oil.

$N$-(tert-Butoxycarbonyl)-(2S,3R,4S)-3,4-dihydroxyproline Benzyl Ester (19a) (Table 2, Entry 1). To a solution of alkene 25a $(3.60 \mathrm{~g}, 11.9 \mathrm{mmol})$ in dioxane $(60.0 \mathrm{~mL})$ and water $(15.0 \mathrm{~mL})$ were added NMO (3.48 g, $29.7 \mathrm{mmol}$ ) and $\mathrm{OsO}_{4}\left(4 \mathrm{wt} \%\right.$ in $\mathrm{H}_{2} \mathrm{O}, 0.5$ $\mathrm{mL}$ ). After the mixture was stirred for 2 days at room temperature, TLC analysis indicated complete conversion of the starting material. The mixture was quenched with a saturated aqueous solution of $\mathrm{Na}_{2} \mathrm{~S}_{2} \mathrm{O}_{3}(40 \mathrm{~mL})$ and stirred at room temperature for $15 \mathrm{~min}$. Next, the aqueous layer was extracted with EtOAc $(4 \times 100 \mathrm{~mL})$. The combined organic phases were washed with a saturated aqueous solution of $\mathrm{NaHCO}_{3}(100 \mathrm{~mL})$ and brine $(100 \mathrm{~mL})$, dried over $\mathrm{MgSO}_{4}$, and evaporated in vacuo. Purification by flash chromatography (hexane/acetone 70:30) yielded diol 19a (3.69 g, 92\%) as a clear oil. Data correspond to literature data. ${ }^{34}$

Reaction with $A D-M i x-\alpha$ (Table 2, Entry 3). To a solution of alkene $25 \mathrm{a}(280.0 \mathrm{mg}, 0.923 \mathrm{mmol})$ in $t-\mathrm{BuOH}(3.0 \mathrm{~mL})$ and water $(3.0 \mathrm{~mL})$ were added $\mathrm{AD}$-mix- $\alpha(1.29 \mathrm{~g})$ and $\mathrm{CH}_{3} \mathrm{SO}_{2} \mathrm{NH}_{2}(87.8 \mathrm{mg}$, $0.923 \mathrm{mmol})$. After 3 days, the mixture was quenched with a saturated aqueous solution of $\mathrm{Na}_{2} \mathrm{~S}_{2} \mathrm{O}_{3}(10 \mathrm{~mL})$ and stirred at room temperature for $15 \mathrm{~min}$. Next, the aqueous layer was extracted with EtOAc $(4 \times 15 \mathrm{~mL})$. The combined organic phases were washed with a saturated aqueous solution of $\mathrm{NaHCO}_{3}(15 \mathrm{~mL})$, dried over $\mathrm{MgSO}_{4}$, and evaporated in vacuo. Purification by HPLC (hexane/ acetone $75: 25)$ yielded diol $19 \mathrm{a}(256.0 \mathrm{mg}, 82 \%)$ as a clear oil. Data correspond to literature data. ${ }^{34}$

Reaction with $A D-M i x-\beta$ (Table 2, Entry 4). To a solution of alkene $25 \mathrm{a}(203.2 \mathrm{mg}, 0.670 \mathrm{mmol})$ in $t-\mathrm{BuOH}(3.0 \mathrm{~mL})$ and water $(3.0 \mathrm{~mL})$ were added $\mathrm{AD}-\mathrm{mix}-\beta(938.0 \mathrm{mg})$ and $\mathrm{CH}_{3} \mathrm{SO}_{2} \mathrm{NH}_{2}(63.7$ $\mathrm{mg}, 0.670 \mathrm{mmol})$. After 7 days, the mixture was quenched with a saturated aqueous solution of $\mathrm{Na}_{2} \mathrm{~S}_{2} \mathrm{O}_{3}(10 \mathrm{~mL})$ and stirred at room temperature for $15 \mathrm{~min}$. Next, the aqueous layer was extracted with EtOAc $(4 \times 15 \mathrm{~mL})$. The combined organic phases were washed with a saturated aqueous solution of $\mathrm{NaHCO}_{3}(15 \mathrm{~mL})$, dried over $\mathrm{MgSO}_{4}$, and evaporated in vacuo. Purification by HPLC (hexane/ acetone $75: 25)$ yielded diol 19a (150.1 mg, 66\%) as a clear oil. Data correspond to literature data. ${ }^{34}$

$N$-(9-Fluorenylmethyloxycarbonyl)-(2S,3R,4S)-3,4-dihydroxyproline Benzyl Ester (19c) (Table 2, Entry 5). To a solution of alkene $25 \mathrm{c}(645.2 \mathrm{mg}, 1.516 \mathrm{mmol})$ in acetone $(4.5 \mathrm{~mL})$ and water $(1.5 \mathrm{~mL})$ were added $\mathrm{NMO}(444.1 \mathrm{mg}, 3.791 \mathrm{mmol})$ and $\mathrm{K}_{2} \mathrm{OsO}_{4}$. $2 \mathrm{H}_{2} \mathrm{O}(20.0 \mathrm{mg}, 0.054 \mathrm{mmol})$. After the mixture was stirred for $14 \mathrm{~h}$ at room temperature, TLC analysis indicated complete conversion of the starting material. The mixture was quenched with a saturated aqueous solution of $\mathrm{Na}_{2} \mathrm{~S}_{2} \mathrm{O}_{3}(10 \mathrm{~mL})$ and stirred at room temperature for $1 \mathrm{~h}$. Next, the aqueous layer was extracted with EtOAc $(4 \times 15 \mathrm{~mL})$, and the combined organic phases were washed with brine $(20 \mathrm{~mL})$, dried over $\mathrm{MgSO}_{4}$, and evaporated in vacuo. Purification by flash chromatography (hexane/acetone 70:30) yielded diol 19c as a white solid (553.8 mg, 80\%): ${ }^{1} \mathrm{H}$ NMR (500 MHz, $\left.\mathrm{CDCl}_{3}\right)(51: 49$ rotamer ratio) $\delta 7.82-7.20(\mathrm{~m}, 13 \mathrm{H}$ major $\mathrm{Ar}-\mathrm{H}+$ $13 \mathrm{H}$ minor $\mathrm{Ar}-\mathrm{H}$ ), $5.24\left(\mathrm{~d}, J=12.4 \mathrm{~Hz}, 1 \mathrm{H}\right.$, major $\left.\mathrm{CHH}^{\prime} \mathrm{Ph}\right), 5.18$ $\left(\mathrm{d}, J=12.4 \mathrm{~Hz}, 1 \mathrm{H}\right.$, major $\left.\mathrm{CH}^{\prime} \mathrm{Ph}\right), 5.14(\mathrm{~d}, J=12.2 \mathrm{~Hz}, 1 \mathrm{H}$, minor $\left.\mathrm{CHH}^{\prime} \mathrm{Ph}\right), 5.08\left(\mathrm{~d}, J=12.2 \mathrm{~Hz}, 1 \mathrm{H}, \operatorname{minor} \mathrm{CHH}^{\prime} \mathrm{Ph}\right), 4.59-4.21(\mathrm{~m}$, $1 \mathrm{H}$ major $\mathrm{C}_{\alpha} \mathrm{H}+1 \mathrm{H}$ minor $\mathrm{C}_{\alpha} \mathrm{H}+1 \mathrm{H}$ major $\mathrm{C}_{\beta} \mathrm{H}+1 \mathrm{H}$ minor $\mathrm{C}_{\beta} \mathrm{H}$ $+1 \mathrm{H}$ major $\mathrm{C}_{\gamma} \mathrm{H}+1 \mathrm{H}$ minor $\mathrm{C}_{\gamma} \mathrm{H}+2 \mathrm{H}$ major $\mathrm{NCO}_{2}-\mathrm{CH}_{2}-\mathrm{CH}+$ $2 \mathrm{H}$ minor $\mathrm{NCO}_{2}-\mathrm{CH}_{2}-\mathrm{CH}+1 \mathrm{H}$ major $\left.\mathrm{NCO}_{2}-\mathrm{CH}_{2}-\mathrm{CH}\right), 3.96(\mathrm{t}$, $J=6.9 \mathrm{~Hz}, 1 \mathrm{H}$ minor $\mathrm{NCO}_{2}-\mathrm{CH}_{2}-\mathrm{CH}$ ), $3.85-3.53$ (m, $2 \mathrm{H}$ major $\mathrm{C}_{\delta} \mathrm{H}_{2}+2 \mathrm{H}$ minor $\mathrm{C}_{\delta} \mathrm{H}_{2}$ ), 3.29 (br d, $J=5.0 \mathrm{~Hz}, 1 \mathrm{H}$ minor $\mathrm{OH}$ ), 3.19 (br d, $J=5.5 \mathrm{~Hz}, 1 \mathrm{H}$ major $\mathrm{OH}), 2.91(\mathrm{br} \mathrm{d}, J=4.6 \mathrm{~Hz}, 1 \mathrm{H}$ minor $\left.\mathrm{O}^{\prime} \underline{\mathrm{H}^{\prime}}\right), 2.85$ (br d, $J=5.3 \mathrm{~Hz}, 1 \mathrm{H}$ major $\left.\mathrm{O}^{\prime} \underline{\mathrm{H}^{\prime}}\right) \mathrm{ppm} ;{ }^{13} \mathrm{C}\left\{{ }^{1} \mathrm{H}\right\} \mathrm{NMR}$ $\left(126 \mathrm{MHz}, \mathrm{CDCl}_{3}\right)\left(51: 49\right.$ rotamer ratio) $\delta 170.7$ (minor $\mathrm{C}_{\alpha}-\mathrm{CO}_{2}$ ), 
170.6 (major $\mathrm{C}_{\alpha}-\underline{\mathrm{CO}}_{2}$ ), 155.0 (major $\mathrm{NCO}_{2}$ ), 154.6 (minor $\mathrm{NCO}_{2}$ ), $143.9+143.8+143.7+143.5$ (major and minor overlap, $\mathrm{C}_{q}$, Fmoc), $141.3+141.24+141.15$ (major and minor overlap, $\mathrm{C}_{\mathrm{q}, \mathrm{Fmoc}}$ ), 135.2 (major $\underline{\mathrm{C}}_{\mathrm{q}, \mathrm{Ph}}$ ), $135.1\left(\right.$ minor $\left.\underline{\mathrm{C}}_{\mathrm{q}, \mathrm{Ph}}\right), 128.59+128.56+128.48+128.4$ $+128.3+128.1+127.74+127.71+127.6+127.07+127.04+$ $125.09+125.05+124.9+120.0+119.91+119.89$ (major and minor overlap, $\mathrm{CH}_{\mathrm{Ar}}$ ), 75.8 (minor $\mathrm{C}_{\beta} / \mathrm{C}_{\gamma}$ ), 74.6 (major $\mathrm{C}_{\beta} / \mathrm{C}_{\gamma}$ ), 70.5 (major $\mathrm{C}_{\beta} / \mathrm{C}_{\gamma}$ ), 69.7 (minor $\mathrm{C}_{\beta} / \mathrm{C}_{\gamma}$ ), 67.8 (major and minor overlap, $\mathrm{NCO}_{2}-$ $\underline{\mathrm{C}} \mathrm{H}_{2}-\mathrm{CH}$ ), $67.4\left(\right.$ major $\left.\underline{\mathrm{CH}}_{2} \mathrm{Ph}\right), 67.3$ (minor $\left.\mathrm{CH}_{2} \mathrm{Ph}\right), 64.9$ (major $\mathrm{C}_{\alpha}$ ), 64.6 (minor $\mathrm{C}_{\alpha}$ ), 51.2 (minor $\mathrm{C}_{\delta}$ ), 50.8 (major $\mathrm{C}_{\delta}$ ), 47.1 (major $\mathrm{NCO}_{2}-\mathrm{CH}_{2}-\underline{\mathrm{CH}}$ ), 47.0 (minor $\mathrm{NCO}_{2}-\mathrm{CH}_{2}-\underline{\mathrm{CH}}$ ) ppm; $R_{f} 0.21$ (hexane/acetone 70:30); $[\alpha]_{\mathrm{D}}^{22}-184.1\left(c 1.2, \mathrm{CHCl}_{3}\right) ; \mathrm{MS}$ (ESI) $(\mathrm{m} /$ z) $460.4[\mathrm{M}+\mathrm{H}]^{+}, 482.4[\mathrm{M}+\mathrm{Na}]^{+}$; HRMS (ESI) for $\mathrm{C}_{27} \mathrm{H}_{25} \mathrm{NNaO}_{6}[\mathrm{M}+\mathrm{Na}]^{+}$calcd for 482.1574 , found 482.1575; IR 3412 (br m), 1743(s), 1681 (s), 1451 (m), 1424 (s), 1185 (s), 738 (s) $\mathrm{cm}^{-1}$.

( \pm )- $N$-(tert-Butoxycarbonyl)-(2R,3S,4R)-3,4-difluoroproline Benzyl Ester (20a) (Scheme 7). To a solution of diol ( \pm )-19a $(950.0 \mathrm{mg}, 2.816 \mathrm{mmol})$ in DCM $(8.0 \mathrm{~mL})$ at $0{ }^{\circ} \mathrm{C}$ was added DAST $(3.72 \mathrm{~mL}, 28.16 \mathrm{mmol})$ dropwise. After the mixture was stirred at room temperature for $22 \mathrm{~h}$, an extra portion of DAST ( $2.26 \mathrm{~g}$, 14.08 $\mathrm{mmol}$ ) was added. After another $32 \mathrm{~h}$ of stirring at room temperature, the reaction mixture was cooled to $0{ }^{\circ} \mathrm{C}$ and quenched by dropwise addition of a saturated aqueous solution of $\mathrm{NaHCO}_{3}(30 \mathrm{~mL})$. Next, the aqueous layer was extracted with DCM $(5 \times 30 \mathrm{~mL})$, and the combined organic phases were washed with brine $(50 \mathrm{~mL})$, dried over $\mathrm{MgSO}_{4}$, and evaporated in vacuo. Purification by flash chromatography (hexane/EtOAc 70:30) yielded difluoroproline $( \pm$ )-20a and a cyclic sulphite side product as an inseparable mixture. This mixture was dissolved in water $(5.0 \mathrm{~mL})$ and acetonitrile $(7.0 \mathrm{~mL})$, and sodium periodate $(278.9 \mathrm{mg}, 1.304 \mathrm{mmol})$ and a catalytic amount of ruthenium(III) chloride were added. After $3 \mathrm{~h}$, a saturated aqueous solution of $\mathrm{NaHCO}_{3}(10 \mathrm{~mL})$ was added and the aqueous layer was extracted with DCM $(3 \times 30 \mathrm{~mL})$. Next, the combined organic phases were dried over $\mathrm{MgSO}_{4}$ and evaporated in vacuo. Purification via HPLC (hexane/EtOAc 80:20) yielded difluoroproline ( \pm )-20a (248.1 mg, 26\%) as a clear oil, which spontaneously crystallized upon standing. Data correspond to literature data. ${ }^{34}$

$N$-(9-Fluorenylmethyloxycarbonyl)-(2R,3S,4R)-3,4-difluoroproline Benzyl Ester (20c) (Scheme 7). To a solution of diol 19c $(155.7 \mathrm{mg}, 0.339 \mathrm{mmol})$ in DCM $(3.0 \mathrm{~mL})$ at $0{ }^{\circ} \mathrm{C}$ was added DAST $(0.224 \mathrm{~mL}, 1.694 \mathrm{mmol})$ dropwise. After stirring at room temperature for $16 \mathrm{~h}$, an extra portion of DAST $(0.224 \mathrm{~mL}, 1.694 \mathrm{mmol})$ was added. After another $50 \mathrm{~h}$ of stirring at room temperature, the reaction mixture was cooled to $0{ }^{\circ} \mathrm{C}$, diluted with DCM $(10 \mathrm{~mL})$, and quenched by dropwise addition of a saturated aqueous solution of $\mathrm{NaHCO}_{3}(20 \mathrm{~mL})$. Next, the aqueous layer was extracted with DCM $(3 \times 20 \mathrm{~mL})$, and the combined organic phases were washed with brine $(20 \mathrm{~mL})$, dried over $\mathrm{MgSO}_{4}$, and evaporated in vacuo. Purification by flash chromatography (hexane/acetone 80:20) yielded difluoroproline 20c and a cyclic sulphite side product as an inseparable mixture. This mixture was dissolved in water $(1.0 \mathrm{~mL})$ and acetonitrile $(1.0 \mathrm{~mL})$, and sodium periodate $(16.9 \mathrm{mg}, 0.079$ mmol) and a catalytic amount of ruthenium(III) chloride were added. After $3 \mathrm{~h}$, a saturated aqueous solution of $\mathrm{NaHCO}_{3}(7 \mathrm{~mL})$ was added and the aqueous layer was extracted with DCM $(4 \times 10 \mathrm{~mL})$. Next, the combined organic phases were dried over $\mathrm{MgSO}_{4}$ and evaporated in vacuo. Purification via HPLC (hexane/EtOAc 80:20) yielded difluoroproline $20 \mathrm{c}(21.4 \mathrm{mg}, 14 \%)$ as a clear oil: ${ }^{1} \mathrm{H}$ NMR (500 $\mathrm{MHz}, \mathrm{CDCl}_{3}$ ) (51:49 rotamer ratio) $\delta 7.82-7.24(\mathrm{~m}, 13 \mathrm{H}$ major $\mathrm{Ar}-$ $\mathrm{H}+13 \mathrm{H}$ minor $\mathrm{Ar}-\mathrm{H}), 5.30\left(\mathrm{~d}, J=12.4 \mathrm{~Hz}, 1 \mathrm{H}\right.$, major $\left.\mathrm{CHH}^{\prime} \mathrm{Ph}\right)$, $5.22\left(\mathrm{~d}, J=12.4 \mathrm{~Hz}, 1 \mathrm{H}\right.$, major $\left.\mathrm{CH}_{\mathrm{H}^{\prime}} \mathrm{Ph}\right), 5.19(\mathrm{~d}, J=12.4 \mathrm{~Hz}, 1 \mathrm{H}$, minor $\left.\mathrm{CHH}^{\prime} \mathrm{Ph}\right), 5.08\left(\mathrm{~d}, J=12.5 \mathrm{~Hz}, 1 \mathrm{H}\right.$, minor $\left.\mathrm{CH}^{\prime} \mathrm{Ph}\right), 5.35-$ $4.95\left(\mathrm{~m}, 1 \mathrm{H}\right.$ major $\mathrm{C}_{\beta} \mathrm{H}+1 \mathrm{H}$ minor $\mathrm{C}_{\beta} \mathrm{H}+1 \mathrm{H}$ major $\mathrm{C}_{\gamma} \mathrm{H}+1 \mathrm{H}$ minor $\left.\mathrm{C}_{\gamma} \mathrm{H}\right), 4.74-4.33\left(\mathrm{~m}, 1 \mathrm{H}\right.$ major $\mathrm{C}_{\alpha} \mathrm{H}+1 \mathrm{H}$ minor $\mathrm{C}_{\alpha} \mathrm{H}+2 \mathrm{H}$ major $\mathrm{NCO}_{2}-\mathrm{CH}_{2}-\mathrm{CH}+2 \mathrm{H}$ minor $\left.\mathrm{NCO}_{2}-\mathrm{CH}_{2}-\mathrm{CH}\right), 4.26(\mathrm{t}, J=$ $6.7 \mathrm{~Hz}, 1 \mathrm{H}$ major $\left.\mathrm{NCO}_{2}-\mathrm{CH}_{2}-\mathrm{C} \underline{\mathrm{H}}\right), 4.03(\mathrm{t}, J=6.4 \mathrm{~Hz}, 1 \mathrm{H}$ minor $\left.\mathrm{NCO}_{2}-\mathrm{CH}_{2}-\mathrm{CH}\right), 4.01-3.76\left(\mathrm{~m}, 2 \mathrm{H}\right.$ major $\mathrm{C}_{\delta} \mathrm{H}_{2}+2 \mathrm{H}$ minor $\mathrm{C}_{\delta} \mathrm{H}_{2}$ ) ppm; ${ }^{13} \mathrm{C}\left\{{ }^{1} \mathrm{H}\right\}$ NMR $\left(126 \mathrm{MHz}, \mathrm{CDCl}_{3}\right.$ ) (51:49 rotamer ratio) $\delta 166.1\left(\mathrm{~d}, J=5.5 \mathrm{~Hz}\right.$, minor $\left.\mathrm{C}_{\alpha}-\underline{\mathrm{CO}}_{2}\right), 165.9(\mathrm{~d}, J=6.4 \mathrm{~Hz}$, major $\mathrm{C}_{\alpha}-\underline{\mathrm{CO}}_{2}$ ), 154.4 (major $\mathrm{NCO}_{2}$ ), 154.0 (minor $\mathrm{NCO}_{2}$ ), 143.85 $+143.84+143.4+143.3$ (major and minor overlap, $\mathrm{C}_{\mathrm{q}, \mathrm{Fmoc}}$ ), $141.4+$ $141.3+141.24+141.18$ (major and minor overlap, $\mathrm{C}_{\mathrm{q}, \mathrm{Fmoc}}$ ), 135.1 (major $\underline{\mathrm{C}}_{\mathrm{q}, \mathrm{Ph}}$ ), 135.0 (minor $\underline{\mathrm{C}}_{\mathrm{q}, \mathrm{Ph}}$ ), $128.51+128.50+128.40+$ $128.35+128.26+128.20+127.82+127.79+127.7+127.14+$ $127.12+127.09+127.06+125.0+124.9+124.83+124.77+$ $120.02+199.99+119.95$ (major and minor overlap, $\underline{\mathrm{C}} \mathrm{H}_{\mathrm{Ar}}$ ), $88.9(\mathrm{dd}$, $J=199.8,15.4 \mathrm{~Hz}$, minor $\left.\mathrm{C}_{\beta} / \mathrm{C}_{\gamma}\right), 88.2(\mathrm{dd}, J=198.0,15.4 \mathrm{~Hz}$, major $\left.\mathrm{C}_{\beta} / \mathrm{C}_{\gamma}\right), 87.3\left(\mathrm{dd}, J=197.1,16.4 \mathrm{~Hz}\right.$, major $\left.\mathrm{C}_{\beta} / \mathrm{C}_{\gamma}\right), 86.8(\mathrm{dd}, J=$ 195.3, $15.4 \mathrm{~Hz}$, minor $\mathrm{C}_{\beta} / \mathrm{C}_{\gamma}$ ), 67.9 (minor $\mathrm{NCO}_{2}-\mathrm{CH}_{2}-\mathrm{CH}$ ), 67.8 (major $\mathrm{NCO}_{2}-\mathrm{CH}_{2}-\mathrm{CH}$ ), 67.63 (major $\mathrm{CH}_{2} \mathrm{Ph}$ ), 67.55 (minor $\left.\mathrm{CH}_{2} \mathrm{Ph}\right), 60.4\left(\mathrm{~d}, J=21.8 \mathrm{~Hz}\right.$, major $\left.\mathrm{C}_{\alpha}\right), 60.0(\mathrm{~d}, J=21.8 \mathrm{~Hz}$, minor $\left.\mathrm{C}_{\alpha}\right), 48.0\left(\mathrm{dd}, J=25.4,1.8 \mathrm{~Hz}\right.$, minor $\left.\mathrm{C}_{\delta}\right), 47.6(\mathrm{dd}, J=26.3,1.8 \mathrm{~Hz}$, major $\mathrm{C}_{\delta}$ ), 47.06 (major $\mathrm{NCO}_{2}-\mathrm{CH}_{2}-\underline{\mathrm{CH}}$ ), 47.04 (minor $\mathrm{NCO}_{2}-$ $\mathrm{CH}_{2}-\mathrm{CH}$ ) ppm; ${ }^{19} \mathrm{~F}$ NMR $\left(376 \mathrm{MHz}, \mathrm{CDCl}_{3}\right)$ (51:49 rotamer ratio) $\delta-204.6$ to $-204.9(\mathrm{~m}, 1 \mathrm{~F}$, minor $\mathrm{F}),-205.1$ to $-205.4(\mathrm{~m}, 1 \mathrm{~F}$, major F), -207.6 to $-208.0\left(\mathrm{~m}, 1 \mathrm{~F}\right.$, minor $\left.\mathrm{F}^{\prime}\right),-208.3$ to $-208.6(\mathrm{~m}$, $1 \mathrm{~F}$, major $\left.\mathrm{F}^{\prime}\right) \mathrm{ppm}^{19}{ }^{19} \mathrm{~F}\left\{{ }^{1} \mathrm{H}\right\}$ NMR (376 $\left.\mathrm{MHz}, \mathrm{CDCl}_{3}\right) \quad(51: 49$ rotamer ratio) $\delta-204.7$ (br d, $J=5.2 \mathrm{~Hz}, 1 \mathrm{~F}$, minor $\mathrm{F}$ ), $-205.2(\mathrm{br} \mathrm{d}$, $J=5.2 \mathrm{~Hz}, 1 \mathrm{~F}$, major $\mathrm{F}),-207.7\left(\mathrm{br} \mathrm{d}, J=3.5 \mathrm{~Hz}, 1 \mathrm{~F}\right.$, minor $\left.\mathrm{F}^{\prime}\right)$, -208.4 (br d, $J=5.2 \mathrm{~Hz}, 1 \mathrm{~F}$, major $\mathrm{F}^{\prime}$ ) ppm; $R_{f} 0.35$ (hexane/EtOAc 75:25); $[\alpha]_{\mathrm{D}}^{24}-46.4\left(c 0.9, \mathrm{CHCl}_{3}\right) ; \mathrm{MS}(\mathrm{ESI})(\mathrm{m} / z) 464.1[\mathrm{M}+$ $\mathrm{H}]^{+}$; HRMS (ESI) for $\mathrm{C}_{27} \mathrm{H}_{23} \mathrm{~F}_{2} \mathrm{NNaO}_{4}[\mathrm{M}+\mathrm{Na}]^{+}$calcd for 486.1487, found 486.1481; IR $1756(\mathrm{~m}), 1708(\mathrm{~s}), 1450(\mathrm{~m}), 1416$ (s), $1176(\mathrm{~s}), 1100(\mathrm{~s}), 736(\mathrm{~s}) \mathrm{cm}^{-1}$.

$\mathrm{N}$-(tert-Butoxycarbonyl)-(2S)-4-oxoproline Benzyl Ester (29a). To a solution of $26 \mathrm{a}(4.40 \mathrm{~g}, 13.69 \mathrm{mmol})$ in $\mathrm{CH}_{2} \mathrm{Cl}_{2}(40$ $\mathrm{mL})$ was added Dess-Martin periodinane $(6.39 \mathrm{~g}, 15.06 \mathrm{mmol})$ in three portions over $15 \mathrm{~min}$, and the solution was stirred at rt. After $105 \mathrm{~min}$, the reaction mixture was quenched with a saturated aqueous solution of $\mathrm{Na}_{2} \mathrm{~S}_{2} \mathrm{O}_{3}(30 \mathrm{~mL})$ and a saturated aqueous solution of $\mathrm{NaHCO}_{3}(30 \mathrm{~mL})$. The aqueous phase was extracted with $\mathrm{CH}_{2} \mathrm{Cl}_{2}(3$ $\times 40 \mathrm{~mL})$. The combined organic phases were dried over $\mathrm{MgSO}_{4}$, and the solvent was evaporated in vacuo. Purification by flash chromatography using a Biotage purification system (hexane/acetone gradient) yielded ketone 29a (4.12 g, 94\%) as a clear oil: ${ }^{1} \mathrm{H}$ NMR $\left(400 \mathrm{MHz}, \mathrm{CDCl}_{3}\right)(52: 48$ rotamer ratio) $\delta 7.41-7.30(\mathrm{~m}, 5 \mathrm{H}$ major $\mathrm{Ph}+5 \mathrm{H}$ minor $\mathrm{Ph}), 5.30-5.07\left(\mathrm{~m}, 2 \mathrm{H}\right.$ major $\mathrm{CH}_{2}-\mathrm{Ph}+2 \mathrm{H}$ minor $\mathrm{CH}_{2}-\mathrm{Ph}$ ), $4.87\left(\right.$ br d, $J=9.9 \mathrm{~Hz}, 1 \mathrm{H}$, major $\left.\mathrm{C}_{\alpha} \mathrm{H}\right), 4.74($ br d, $\mathrm{J}=$ $10.6 \mathrm{~Hz}, 1 \mathrm{H}$, minor $\left.\mathrm{C}_{\alpha} \mathrm{H}\right), 3.99-3.82\left(\mathrm{~m}, 2 \mathrm{H}\right.$ minor $\mathrm{C}_{\delta} \mathrm{H} 2+2 \mathrm{H}$ major $\left.\mathrm{C}_{\delta} \mathrm{H} 2\right)$, 3.02-2.84 (m, $1 \mathrm{H}$ major $\mathrm{C}_{\beta} \mathrm{HH}^{\prime}+1 \mathrm{H}$ minor $\left.\mathrm{C}_{\beta} \mathrm{HH}^{\prime}\right)$, 2.64-2.50 (m, 1H major $\mathrm{C}_{\beta} \mathrm{HH}^{\prime}+1 \mathrm{H}$ minor $\left.\mathrm{C}_{\beta} \mathrm{HH}^{\prime}\right), 1.48(\mathrm{~s}, 9 \mathrm{H}$, minor $\left.\mathrm{CO}_{2} \mathrm{C}\left(\mathrm{CH}_{3}\right)_{3}\right)$, 1.38 (s, 9H, major $\left.\mathrm{CO}_{2} \mathrm{C}\left(\mathrm{CH}_{3}\right)_{3}\right)$ ppm; ${ }^{13} \mathrm{C}\left\{{ }^{1} \mathrm{H}\right\}$ NMR $\left(100 \mathrm{MHz}, \mathrm{CDCl}_{3}\right)$ (52:48 rotamer ratio) $\delta 208.4$ (minor $\mathrm{C}_{\gamma}$ ), 207.6 (major $\mathrm{C}_{\gamma}$ ), 171.6 (major and minor overlap, $\mathrm{C}_{\alpha^{-}}$ $\mathrm{CO}_{2}$ ), 154.2 (minor $\left.\mathrm{CO}_{2} \mathrm{C}\left(\mathrm{CH}_{3}\right)_{3}\right), 153.8$ (major $\mathrm{CO}_{2} \mathrm{C}\left(\mathrm{CH}_{3}\right)_{3}$ ), 135.1 (minor $\left.\mathrm{C}_{\mathrm{q}, \mathrm{Ph}}\right), 135.0$ (major $\left.\mathrm{C}_{\mathrm{q}, \mathrm{Ph}}\right), 128.72+128.66+128.5+$ 128.2 (major and minor overlap, $\mathrm{CH}_{\mathrm{Ph}}$ ), 81.3 (major and minor overlap, $\left.\mathrm{CO}_{2} \mathrm{C}\left(\mathrm{CH}_{3}\right)_{3}\right), 67.4$ (major and minor overlap, $\mathrm{CH}_{2} \mathrm{Ph}$ ), 53.4 (major $\mathrm{C}_{\alpha}$ ), 55.7 (minor $\mathrm{C}_{\alpha}$ ), 52.9 (minor $\mathrm{C}_{\delta}$ ), 52.5 (major $\mathrm{C}_{\delta}$ ), 41.2 (major $\mathrm{C}_{\beta}$ ), 40.7 (minor $\mathrm{C}_{\beta}$ ), 28.3 (minor $\mathrm{CO}_{2} \mathrm{C}\left(\mathrm{CH}_{3}\right)_{3}$ ), 28.1 (major $\left.\mathrm{CO}_{2} \mathrm{C}\left(\mathrm{CH}_{3}\right)_{3}\right)$ ppm; $R_{f} 0.35$ (hexane/acetone 80:20); $[\alpha]_{\mathrm{D}}^{22}$ -16.8 (c 1.0, $\left.\mathrm{CHCl}_{3}\right)$. Data is consistent with literature data. ${ }^{65}$

$\mathrm{N}$-(tert-Butoxycarbonyl)-(2R,3S,4R)-3-fluoro-4-hydroxyproline Benzyl Ester (50a) and $\mathrm{N}$-(tert-Butoxycarbonyl)(2R,3R,4S)-3-fluoro-4-hydroxyproline Benzyl Ester (51a) (Scheme 8). To a solution of diisopropylamine $(2.56 \mathrm{~mL}, 18.23$ mmol) in THF $(50.0 \mathrm{~mL})$ at $-78{ }^{\circ} \mathrm{C}$ was added $n$-BuLi $(2.5 \mathrm{M}$ in hexanes, $6.81 \mathrm{~mL}, 17.02 \mathrm{mmol}$ ) dropwise, and the mixture was stirred for $20 \mathrm{~min}$. A precooled solution of ketone $29 \mathrm{a}(3.88 \mathrm{~g}, 12.16 \mathrm{mmol})$ in THF $(50.0 \mathrm{~mL})$ was cannulated into the mixture and was then stirred for $30 \mathrm{~min}$ at $-78{ }^{\circ} \mathrm{C}$. Next, TMSCl $(3.09 \mathrm{~mL}, 24.32 \mathrm{mmol})$ was added, and the mixture was allowed to warm to room temperature. After $1 \mathrm{~h}$, the solvent was removed in vacuo. The residue was redissolved in EtOAc $(50.0 \mathrm{~mL})$, and the residual solids were removed via filtration. The solvent was again removed in vacuo. The crude product was redissolved in acetonitrile $(80.0 \mathrm{~mL})$, and Selectfluor $(10.77 \mathrm{~g}, 30.40 \mathrm{mmol})$ was added. After the mixture was stirred at room temperature for $16 \mathrm{~h}$, the solvent was removed in vacuo. The residue was dissolved in water $(60 \mathrm{~mL})$ and extracted with 
EtOAc $(4 \times 60 \mathrm{~mL})$. The combined organic phases were dried over $\mathrm{MgSO}_{4}$ and evaporated in vacuo. Purification by flash chromatography (hexane/acetone 80:20) yielded a mixture of diastereomeric fluoroketones $27 \mathrm{a} / \mathbf{2 8} \mathrm{a}(1.26 \mathrm{~g}, 31 \%)$. Subsequently, the diastereomeric mixture $(1.10 \mathrm{~g}, 3.26 \mathrm{mmol})$ was dissolved in THF $(20.0 \mathrm{~mL})$ and methanol $(4.0 \mathrm{~mL})$ and was cooled to $0{ }^{\circ} \mathrm{C}$, and sodium borohydride $(185.0 \mathrm{mg}, 4.89 \mathrm{mmol})$ was added in one portion. After $3 \mathrm{~h}$, the reaction was quenched with a saturated aqueous solution of $\mathrm{NaHCO}_{3}(20 \mathrm{~mL})$ and the aqueous phase was extracted with EtOAc $(4 \times 30 \mathrm{~mL})$. The combined organic phases were dried over $\mathrm{MgSO}_{4}$ and evaporated in vacuo. Purification by flash chromatography (hexane/acetone 85:15 to $60: 40$ and hexane/EtOAc 80:20 to 60:40) yielded the fluorohydrins 50a ( $460.3 \mathrm{mg}, 40 \%$ (contaminated with an additional $\sim 4 \%$ of 51a)) and 51a $(138.2 \mathrm{mg}, 13 \%)$ as clear oils.

Data for N-(tert-Butoxycarbonyl)-(2R,3S,4R)-3-fluoro-4-hydroxyproline Benzyl Ester (50a): ${ }^{1} \mathrm{H}$ NMR $\left(400 \mathrm{MHz}, \mathrm{CDCl}_{3}\right)(60: 40$ rotamer ratio) $\delta 7.42-7.29(\mathrm{~m}, 5 \mathrm{H}$ major $\mathrm{Ph}+5 \mathrm{H}$ minor $\mathrm{Ph}), 5.34$ $\left(\mathrm{d}, J=12.4 \mathrm{~Hz}, 1 \mathrm{H}\right.$, minor $\left.\mathrm{CH}^{\prime} \mathrm{Ph}\right), 5.27(\mathrm{~d}, J=12.2 \mathrm{~Hz}, 1 \mathrm{H}$, major $\left.\mathrm{CHH}^{\prime} \mathrm{Ph}\right), 5.21\left(\mathrm{~d}, J=12.1 \mathrm{~Hz}, 1 \mathrm{H}\right.$, major $\left.\mathrm{CH}^{\prime} \mathrm{Ph}\right), 5.16(\mathrm{~d}, J=$ $12.4 \mathrm{~Hz}, 1 \mathrm{H}$, minor $\left.\mathrm{CH}^{\prime} \mathrm{Ph}\right), 5.27-5.08\left(\mathrm{~m}, 1 \mathrm{H}\right.$ major $\mathrm{C}_{\beta} \mathrm{H}+1 \mathrm{H}$ minor $\left.\mathrm{C}_{\beta} \mathrm{H}\right), 4.64\left(\mathrm{dd}, J=21.0,5.9 \mathrm{~Hz}, 1 \mathrm{H}, \operatorname{minor} \mathrm{C}_{\alpha} \mathrm{H}\right), 4.55(\mathrm{dd}, J$ $=20.7,5.4 \mathrm{~Hz}, 1 \mathrm{H}$, major $\left.\mathrm{C}_{\alpha} \mathrm{H}\right), 4.34-4.21\left(\mathrm{~m}, 1 \mathrm{H}\right.$ major $\mathrm{C}_{\gamma} \mathrm{H}+1 \mathrm{H}$ minor $\left.\mathrm{C}_{\gamma} \mathrm{H}\right), 3.92-3.77\left(\mathrm{~m}, 1 \mathrm{H}\right.$ major $\mathrm{C}_{\delta} \underline{\mathrm{HH}^{\prime}}+1 \mathrm{H}$ minor $\left.\mathrm{C}_{\delta} \underline{\mathrm{H}} \mathrm{H}^{\prime}\right)$, 3.50-3.41 (m, $1 \mathrm{H}$ major $\mathrm{C}_{\delta} \mathrm{H}^{\prime}+1 \mathrm{H}$ minor $\left.\mathrm{C}_{\delta} \mathrm{H}^{\prime}\right)$, 2.83-2.75 (m, $1 \mathrm{H}$ major $\mathrm{OH}+1 \mathrm{H}$ minor $\mathrm{OH}), 1.47\left(\mathrm{~s}, 9 \mathrm{H}\right.$, minor $\left.\mathrm{CO}_{2} \mathrm{C}\left(\mathrm{CH}_{3}\right)_{3}\right)$, 1.33 (s, 9H, major $\left.\mathrm{CO}_{2} \mathrm{C}\left(\mathrm{CH}_{3}\right)_{3}\right)$ ppm; ${ }^{13} \mathrm{C}\left\{{ }^{1} \mathrm{H}\right\}$ NMR $(100 \mathrm{MHz}$, $\left.\mathrm{CDCl}_{3}\right)\left(60: 40\right.$ rotamer ratio) $\delta 168.2\left(\mathrm{~d}, J=6.6 \mathrm{~Hz}\right.$, major $\left.\mathrm{C}_{\alpha}-\mathrm{CO}_{2}\right)$, $167.9\left(\mathrm{~d}, J=7.3 \mathrm{~Hz}\right.$, minor $\left.\mathrm{C}_{\alpha}-\mathrm{CO}_{2}\right), 153.9\left(\right.$ minor $\left.\mathrm{CO}_{2} \mathrm{C}\left(\mathrm{CH}_{3}\right)_{3}\right)$, 153.2 (major $\mathrm{CO}_{2} \mathrm{C}\left(\mathrm{CH}_{3}\right)_{3}$ ), 135.2 (minor $\underline{\mathrm{C}}_{\mathrm{q}, \mathrm{Ph}}$ ), 135.0 (major $\underline{\mathrm{C}}_{\mathrm{q}, \mathrm{Ph}}$ ), $128.63+128.60+128.5+128.3+128.2$ (major and minor overlap, $\left.\underline{\mathrm{CH}}_{\mathrm{Ph}}\right), 91.5\left(\mathrm{~d}, J=189.3 \mathrm{~Hz}\right.$, major $\left.\mathrm{C}_{\beta}\right), 90.8(\mathrm{~d}, J=190.7$ $\mathrm{Hz}$, minor $\mathrm{C}_{\beta}$ ), 81.1 (major $\left.\mathrm{CO}_{2} \mathrm{C}\left(\mathrm{CH}_{3}\right)_{3}\right), 81.0$ (minor $\mathrm{CO}_{2} \mathrm{C}$ $\left.\left(\mathrm{CH}_{3}\right)_{3}\right), 70.6\left(\mathrm{~d}, J=18.3 \mathrm{~Hz}\right.$, minor $\left.\mathrm{C}_{\gamma}\right), 70.1(\mathrm{~d}, J=17.6 \mathrm{~Hz}$, major $\mathrm{C}_{\gamma}$ ), 67.6 (major and minor overlap, $\left.\mathrm{CH}_{2} \mathrm{Ph}\right), 61.7(\mathrm{~d}, J=22.0 \mathrm{~Hz}$, major $\left.\mathrm{C}_{\alpha}\right), 61.3\left(\mathrm{~d}, J=22.0 \mathrm{~Hz}\right.$, minor $\left.\mathrm{C}_{\alpha}\right), 50.5\left(\right.$ minor $\left.\mathrm{C}_{\delta}\right), 49.9$ (major $\mathrm{C}_{\delta}$ ), 28.3 (minor $\left.\mathrm{CO}_{2} \mathrm{C}\left(\mathrm{CH}_{3}\right)_{3}\right), 28.1$ (major $\left.\left.\mathrm{CO}_{2} \mathrm{C}(\underline{\mathrm{CH}})_{3}\right)_{3}\right)$ ppm; $\left.{ }^{19} \mathrm{~F} \mathrm{NMR} \mathrm{(376} \mathrm{MHz,} \mathrm{CDCl}_{3}\right)$ (60:40 rotamer ratio) $\delta-207.3$ (br dt, $J=53.8,20.8 \mathrm{~Hz}, 1 \mathrm{~F}$, major $\mathrm{F}$ ), -207.9 (br dt, $J=54.6,18.6$ $\mathrm{Hz}, 1 \mathrm{~F}$, minor F) ppm; ${ }^{19} \mathrm{~F}\left\{{ }^{1} \mathrm{H}\right\}$ NMR $\left(376 \mathrm{MHz}, \mathrm{CDCl}_{3}\right)(60: 40$ rotamer ratio) $\delta-207.4(\mathrm{~s}, 1 \mathrm{~F}$, major $\mathrm{F}),-208.0(\mathrm{~s}, 1 \mathrm{~F}$, minor $\mathrm{F})$ ppm; $R_{f} 0.30$ (hexane/acetone 70:30); $[\alpha]_{\mathrm{D}}^{22}-29.8\left(\right.$ c 1.5, $\left.\mathrm{CHCl}_{3}\right)$; MS (ESI) $(m / z) 362.3[\mathrm{M}+\mathrm{Na}]^{+}$; HRMS (ESI) for $\mathrm{C}_{17} \mathrm{H}_{22} \mathrm{FNNaO}_{5}$ $[\mathrm{M}+\mathrm{Na}]^{+}$calcd for 362.1374, found 362.1379; IR 3427 (br m), 1760 (s), $1681(\mathrm{~s}), 1400(\mathrm{~m}), 1155$ (s), $1102(\mathrm{~s}) \mathrm{cm}^{-1}$. The NMR data are consistent with literature data. ${ }^{39}$

Data for N-(tert-Butoxycarbonyl)-(2R,3R,4S)-3-fluoro-4-hydroxyproline Benzyl Ester (51a): ${ }^{1} \mathrm{H}$ NMR $\left(400 \mathrm{MHz}, \mathrm{CDCl}_{3}\right)(58: 42$ rotamer ratio) $\delta 7.43-7.28(\mathrm{~m}, 5 \mathrm{H}$ major $\mathrm{Ph}+5 \mathrm{H}$ minor $\mathrm{Ph}), 5.28$ $\left(\mathrm{d}, J=12.4 \mathrm{~Hz}, 1 \mathrm{H}\right.$, minor $\mathrm{C}_{\mathrm{HH}} \mathrm{H}^{\prime} \mathrm{Ph}, 5.21(\mathrm{~d}, J=12.1 \mathrm{~Hz}, 1 \mathrm{H}$, major $\left.\mathrm{CH}^{\prime} \mathrm{Ph}\right), 5.17\left(\mathrm{~d}, J=11.9 \mathrm{~Hz}, 1 \mathrm{H}\right.$, major $\left.\mathrm{CH}^{\prime} \mathrm{Ph}\right), 5.13(\mathrm{~d}, J=$ $12.4 \mathrm{~Hz}, 1 \mathrm{H}$, minor $\mathrm{CH}^{\prime} \mathrm{Ph}$ ), $4.93($ br dm, $J=52.5 \mathrm{~Hz}, 1 \mathrm{H}$ major $\mathrm{C}_{\beta} \mathrm{H}+1 \mathrm{H}$ minor $\left.\mathrm{C}_{\beta} \mathrm{H}\right), 4.61\left(\right.$ br d, $J=21.6 \mathrm{~Hz}, 1 \mathrm{H}$, minor $\left.\mathrm{C}_{\alpha} \mathrm{H}\right)$, $4.49\left(\mathrm{br} \mathrm{dd}, J=21.4,1.1 \mathrm{~Hz}, 1 \mathrm{H}\right.$, major $\left.\mathrm{C}_{\alpha} \mathrm{H}\right), 4.45-4.28(\mathrm{~m}, 1 \mathrm{H}$ major $\mathrm{C}_{\gamma} \mathrm{H}+1 \mathrm{H}$ minor $\left.\mathrm{C}_{\gamma} \mathrm{H}\right), 3.97-3.79\left(\mathrm{~m}, 1 \mathrm{H}\right.$ major $\mathrm{C}_{\delta} \mathrm{HH}^{\prime}+1 \mathrm{H}$ minor $\left.\mathrm{C}_{\delta} \underline{\mathrm{H}} \mathrm{H}^{\prime}\right), 3.40-3.26\left(\mathrm{~m}, 1 \mathrm{H}\right.$ major $\mathrm{C}_{\delta} \mathrm{HH}^{\prime}+1 \mathrm{H}$ minor $\left.\mathrm{C}_{\delta} \mathrm{HH}^{\prime}\right), 2.54-2.41(\mathrm{~m}, 1 \mathrm{H}$ major $\mathrm{OH}+1 \mathrm{H}$ minor $\mathrm{OH}), 1.47(\mathrm{~s}$, $9 \mathrm{H}$, minor $\left.\mathrm{CO}_{2} \mathrm{C}\left(\mathrm{CH}_{3}\right)_{3}\right), 1.33\left(\mathrm{~s}, 9 \mathrm{H}\right.$, major $\left.\mathrm{CO}_{2} \mathrm{C}\left(\mathrm{CH}_{3}\right)_{3}\right)$ ppm; ${ }^{13} \mathrm{C}\left\{{ }^{1} \mathrm{H}\right\} \operatorname{NMR}\left(100 \mathrm{MHz}, \mathrm{CDCl}_{3}\right) \delta 169.1\left(\mathrm{~d}, J=13.2 \mathrm{~Hz}\right.$, major $\mathrm{C}_{\alpha^{-}}$ $\left.\mathrm{CO}_{2}\right), 168.8\left(\mathrm{~d}, J=13.9 \mathrm{~Hz}\right.$, minor $\left.\mathrm{C}_{\alpha}-\mathrm{CO}_{2}\right), 154.1$ (minor $\left.\underline{\mathrm{CO}}_{2} \mathrm{C}\left(\mathrm{CH}_{3}\right)_{3}\right), 153.3$ (major $\left.\underline{\mathrm{CO}}_{2} \mathrm{C}\left(\mathrm{CH}_{3}\right)_{3}\right), 135.1$ (minor $\underline{\mathrm{C}}_{\mathrm{q}, \mathrm{Ph}}$ ), 134.9 (major $\underline{\mathrm{C}}_{\mathrm{q}, \mathrm{Ph}}$ ), $128.7+128.63+128.55+128.5+128.4+128.2$ (major and minor overlap, $\left.\underline{\mathrm{C}} \mathrm{H}_{\mathrm{Ph}}\right), 94.0\left(\mathrm{~d}, J=190.7 \mathrm{~Hz}\right.$, major $\left.\mathrm{C}_{\beta}\right)$, $93.2\left(\mathrm{~d}, J=190.0 \mathrm{~Hz}\right.$, minor $\left.\mathrm{C}_{\beta}\right), 81.0$ (major $\left.\mathrm{CO}_{2} \mathrm{C}\left(\mathrm{CH}_{3}\right)_{3}\right), 80.9$ $\left(\right.$ minor $\left.\mathrm{CO}_{2} \mathrm{C}\left(\mathrm{CH}_{3}\right)_{3}\right), 70.2\left(\mathrm{~d}, J=18.3 \mathrm{~Hz}\right.$, minor $\left.\mathrm{C}_{\gamma}\right), 69.5(\mathrm{~d}, J=$ $17.6 \mathrm{~Hz}$, major $\mathrm{C}_{\gamma}$ ), 67.6 (major and minor overlap, $\underline{\mathrm{CH}}_{2} \mathrm{Ph}$ ), 63.8 (d, $J=23.5 \mathrm{~Hz}$, major $\mathrm{C}_{\alpha}$ ), $63.5\left(\mathrm{~d}, J=24.2 \mathrm{~Hz}\right.$, minor $\mathrm{C}_{\alpha}$ ), 49.7 (minor $\mathrm{C}_{\delta}$ ), 49.2 (major $\mathrm{C}_{\delta}$ ), 28.3 (minor $\mathrm{CO}_{2} \mathrm{C}\left(\mathrm{CH}_{3}\right)_{3}$ ), 28.1 (major $\left.\mathrm{CO}_{2} \mathrm{C}\left(\mathrm{CH}_{3}\right)_{3}\right)$ ppm; ${ }^{19} \mathrm{~F} \mathrm{NMR}\left(376 \mathrm{MHz}, \mathrm{CDCl}_{3}\right)$ (59:41 rotamer ratio) $\delta-199.8(\mathrm{dt}, J=52.0,20.8 \mathrm{~Hz}, 1 \mathrm{~F}$, major $\mathrm{F}),-200.3(\mathrm{dt}, J=$ 52.9, $21.2 \mathrm{~Hz}, 1 \mathrm{~F}$, minor F) ppm, ${ }^{19} \mathrm{~F}\left\{{ }^{1} \mathrm{H}\right\}$ NMR $\left(376 \mathrm{MHz}, \mathrm{CDCl}_{3}\right)$
(58:42 rotamer ratio) $\delta-199.7(\mathrm{~s}, 1 \mathrm{~F}$, major $\mathrm{F}),-200.2(\mathrm{~s}, 1 \mathrm{~F}$, minor F) ppm; $R_{f} 0.32$ (hexane/acetone 70:30); $[\alpha]_{\mathrm{D}}^{22}-18.2\left(c 0.5, \mathrm{CHCl}_{3}\right)$; MS (ESI) $(m / z) 362.4[\mathrm{M}+\mathrm{Na}]^{+}$; HRMS (ESI) for $\mathrm{C}_{17} \mathrm{H}_{22} \mathrm{FNNaO}_{5}$ $[\mathrm{M}+\mathrm{Na}]^{+}$calcd for 362.1374, found 362.1376; IR 3426 (br m), 1749 (s), $1701(\mathrm{~s}), 1404(\mathrm{~m}), 1187(\mathrm{~s}), 1118(\mathrm{~s}) \mathrm{cm}^{-1}$. The NMR data are consistent with literature data. ${ }^{39}$

$N$-(tert-Butoxycarbonyl)-(2R,3S,4S)-3,4-difluoroproline Benzyl Ester (23a) (Scheme 8). To a solution of fluorohydrin 50a (245.7 $\mathrm{mg}, 0.724 \mathrm{mmol}$, including $4 \%$ of $51 \mathrm{a})$ in THF $(6.0 \mathrm{~mL})$ were added tetrabutylammonium difluorotriphenylsilicate $(312.7 \mathrm{mg}$, $0.579 \mathrm{mmol})$, DIPEA $(0.32 \mathrm{~mL}, 1.810 \mathrm{mmol})$, and nonafluorobutanesulfonyl fluoride (NfF) $(0.286 \mathrm{~mL}, 1.593 \mathrm{mmol})$ consecutively. After $18 \mathrm{~h}$, the solvent was removed in vacuo and the crude mixture was purified by flash chromatography (hexane/EtOAc 90:10) to yield difluoroproline 23 a $(190.7 \mathrm{mg}, 78 \%$, including $\sim 4 \%$ of $24 a$ ) as a clear oil: ${ }^{1} \mathrm{H}$ NMR $\left(400 \mathrm{MHz}, \mathrm{CDCl}_{3}\right)$ (60:40 rotamer ratio) $\delta 7.40-7.30$ (m, $5 \mathrm{H}$ major $\mathrm{Ph}+5 \mathrm{H}$ minor $\mathrm{Ph}), 5.43-5.04\left(\mathrm{~m}, 2 \mathrm{H}\right.$ major $\mathrm{C}_{2} \mathrm{Ph}$ $+2 \mathrm{H}$ minor $\mathrm{C}_{2} \mathrm{Ph}+1 \mathrm{H}$ major $\mathrm{C}_{\beta} \mathrm{H}+1 \mathrm{H}$ minor $\mathrm{C}_{\beta} \mathrm{H}+1 \mathrm{H}$ major $\mathrm{C}_{\gamma} \mathrm{H}+1 \mathrm{H}$ minor $\left.\mathrm{C}_{\gamma} \mathrm{H}\right), 4.78\left(\mathrm{dd}, J=26.5,5.1 \mathrm{~Hz}, 1 \mathrm{H}\right.$, minor $\left.\mathrm{C}_{\alpha} \mathrm{H}\right)$, $4.68\left(\mathrm{dd}, J=26.5,4.2 \mathrm{~Hz}, 1 \mathrm{H}\right.$, major $\left.\mathrm{C}_{\alpha} \mathrm{H}\right), 4.05-3.70(\mathrm{~m}, 2 \mathrm{H}$ major $\mathrm{C}_{\delta} \mathrm{H}_{2}+2 \mathrm{H}$ minor $\left.\mathrm{C}_{\delta} \mathrm{H}_{2}\right), 1.49\left(\mathrm{~s}, 9 \mathrm{H}\right.$, minor $\left.\mathrm{CO}_{2} \mathrm{C}\left(\mathrm{CH}_{3}\right)_{3}\right), 1.35$ (s, 9H, major $\left.\mathrm{CO}_{2} \mathrm{C}\left(\mathrm{CH}_{3}\right)_{3}\right)$ ppm; ${ }^{13} \mathrm{C}\left\{{ }^{1} \mathrm{H}\right\}$ NMR $\left(100 \mathrm{MHz}, \mathrm{CDCl}_{3}\right)$ (60:40 rotamer ratio) $\delta 166.7\left(\mathrm{~d}, J=8.8 \mathrm{~Hz}\right.$, major $\left.\mathrm{C}_{\alpha}-\mathrm{CO}_{2}\right), 166.4$ (d, $J=8.8 \mathrm{~Hz}$, minor $\left.\mathrm{C}_{\alpha}-\mathrm{CO}_{2}\right), 154.0\left(\right.$ minor $\left.\mathrm{CO}_{2} \mathrm{C}\left(\mathrm{CH}_{3}\right)_{3}\right), 153.4$ (major $\underline{\mathrm{CO}}_{2} \mathrm{C}\left(\mathrm{CH}_{3}\right)_{3}$ ), 135.2 (minor $\underline{\mathrm{C}}_{\mathrm{q}, \mathrm{Ph}}$ ), 135.1 (major $\underline{\mathrm{C}}_{\mathrm{q}, \mathrm{Ph}}$ ), $128.6+128.53+128.51+128.3+128.2$ (major and minor overlap, $\underline{\mathrm{C}}_{\mathrm{Ph}}$ ), $93.1\left(\mathrm{dd}, J=190.0,33.0 \mathrm{~Hz}\right.$, major $\left.\mathrm{C}_{\beta}\right), 92.2(\mathrm{dd}, J=187.1$, $27.1 \mathrm{~Hz}$, minor $\left.\mathrm{C}_{\beta}\right), 91.9\left(\mathrm{dd}, J=185.6,24.9 \mathrm{~Hz}\right.$, minor $\left.\mathrm{C}_{\gamma}\right), 91.2(\mathrm{dd}$, $J=182.7,30.1 \mathrm{~Hz}$, major $\mathrm{C}_{\gamma}$ ), 81.1 (major and minor overlap, $\left.\mathrm{CO}_{2} \mathrm{C}\left(\mathrm{CH}_{3}\right)_{3}\right), 67.4$ (major and minor overlap, $\mathrm{CH}_{2} \mathrm{Ph}$ ), $62.2(\mathrm{~d}, \mathrm{~J}=$ $21.3 \mathrm{~Hz}$, major $\left.\mathrm{C}_{\alpha}\right), 61.9\left(\mathrm{~d}, J=22.0 \mathrm{~Hz}\right.$, minor $\left.\mathrm{C}_{\alpha}\right), 50.5(\mathrm{~d}, J=22.0$ $\mathrm{Hz}$, minor $\mathrm{C}_{\delta}$ ), $49.9\left(\mathrm{~d}, J=22.7 \mathrm{~Hz}\right.$, major $\mathrm{C}_{\delta}$ ), 28.3 (minor $\left.\mathrm{CO}_{2} \mathrm{C}\left(\mathrm{CH}_{3}\right)_{3}\right), 28.0$ (major $\left.\mathrm{CO}_{2} \mathrm{C}\left(\mathrm{CH}_{3}\right)_{3}\right)$ ppm; ${ }^{19} \mathrm{~F}$ NMR (376 $\left.\mathrm{MHz}, \mathrm{CDCl}_{3}\right)(60: 40$ rotamer ratio) $\delta-192.3$ to $-192.7(\mathrm{~m}, 1 \mathrm{~F}$, minor $\mathrm{F}$ ), -192.8 to -193.2 (m, $1 \mathrm{~F}$, major $\mathrm{F}$ ), -196.2 to -196.9 (m, major $\mathrm{F}^{\prime}+$ minor $\left.\mathrm{F}^{\prime}\right)$ ppm; ${ }^{19} \mathrm{~F}\left\{{ }^{1} \mathrm{H}\right\}$ NMR $\left(376 \mathrm{MHz}, \mathrm{CDCl}_{3}\right)(60: 40$ rotamer ratio $) \delta-192.4(\mathrm{~d}, J=12.1 \mathrm{~Hz}, 1 \mathrm{~F}$, minor $\mathrm{F}),-192.9(\mathrm{~d}, J=$ $12.1 \mathrm{~Hz}, 1 \mathrm{~F}$, major F), $-196.3\left(\mathrm{~d}, J=12.1 \mathrm{~Hz}, 1 \mathrm{~F}\right.$, major $\left.\mathrm{F}^{\prime}\right),-196.6$ (d, $J=12.1 \mathrm{~Hz}, 1 \mathrm{~F}$, minor $\mathrm{F}^{\prime}$ ) ppm; $R_{f} 0.41$ (hexane/acetone 80:20); $[\alpha]_{\mathrm{D}}^{22}-45.0\left(\right.$ c 1.4, $\left.\mathrm{CHCl}_{3}\right)$; MS (ESI) $(\mathrm{m} / z) 342.4[\mathrm{M}+\mathrm{H}]^{+}, 364.4$ $[\mathrm{M}+\mathrm{Na}]^{+}$; HRMS (ESI) for $\mathrm{C}_{17} \mathrm{H}_{21} \mathrm{~F}_{2} \mathrm{NNaO}_{4}[\mathrm{M}+\mathrm{Na}]^{+}$calcd for 364.1331, found 364.1332; IR 1764 (m), 1702 (s), 1394 (s), 1367 (m), $1159(\mathrm{~s}) \mathrm{cm}^{-1}$.

$N$-(tert-Butoxycarbonyl)-(2R,3R,4R)-3,4-difluoroproline Benzyl Ester (24a) (Scheme 8). To a solution of fluorohydrin 51a $(110.0 \mathrm{mg}, \quad 0.324 \mathrm{mmol})$ in THF $(3.0 \mathrm{~mL})$ were added tetrabutylammonium difluorotriphenylsilicate $(140.0 \mathrm{mg}, 0.259$ $\mathrm{mmol})$, DIPEA $(0.141 \mathrm{~mL}, 0.810 \mathrm{mmol})$, and nonafluorobutanesulfonyl fluoride (NfF) $(0.128 \mathrm{~mL}, 0.713 \mathrm{mmol})$ consecutively. After $18 \mathrm{~h}$, the solvent was removed in vacuo and the crude mixture was purified by flash chromatography (hexane/EtOAc 90:10) to yield difluoroproline $24 \mathrm{a}(80.0 \mathrm{mg}, 72 \%)$ as a clear oil: ${ }^{1} \mathrm{H}$ NMR (400 $\left.\mathrm{MHz}, \mathrm{CDCl}_{3}\right)(57: 43$ rotamer ratio) $\delta 7.42-7.29(\mathrm{~m}, 5 \mathrm{H}$ major $\mathrm{Ph}+$ $5 \mathrm{H}$ minor $\mathrm{Ph}), 5.36-5.04\left(\mathrm{~m}, 2 \mathrm{H}\right.$ major $\mathrm{CH}_{2} \mathrm{Ph}+2 \mathrm{H}$ minor $\mathrm{C}_{2} \mathrm{Ph}$ $+1 \mathrm{H}$ major $\mathrm{C}_{\beta} \mathrm{H}+1 \mathrm{H}$ minor $\mathrm{C}_{\beta} \mathrm{H}+1 \mathrm{H}$ major $\mathrm{C}_{\gamma} \mathrm{H}+1 \mathrm{H}$ minor $\left.\mathrm{C}_{\gamma} \mathrm{H}\right), 4.79\left(\mathrm{~d}, J=23.6 \mathrm{~Hz}, 1 \mathrm{H}\right.$, minor $\left.\mathrm{C}_{\alpha} \mathrm{H}\right), 4.63(\mathrm{~d}, J=23.6 \mathrm{~Hz}$, $1 \mathrm{H}$, major $\left.\mathrm{C}_{\alpha} \mathrm{H}\right)$, 3.94-3.75 (m, $2 \mathrm{H}$ major $\mathrm{C}_{\delta} \mathrm{H}_{2}+2 \mathrm{H}$ minor $\left.\mathrm{C}_{\delta} \mathrm{H}_{2}\right)$, $1.49\left(\mathrm{~s}, 9 \mathrm{H}\right.$, minor $\left.\mathrm{CO}_{2} \mathrm{C}\left(\mathrm{CH}_{3}\right)_{3}\right), 1.36\left(\mathrm{~s}, 9 \mathrm{H}\right.$, major $\left.\mathrm{CO}_{2} \mathrm{C}\left(\mathrm{CH}_{3}\right)_{3}\right)$ ppm; ${ }^{13} \mathrm{C}\left\{{ }^{1} \mathrm{H}\right\}$ NMR (100 $\mathrm{MHz}, \mathrm{CDCl}_{3}$ ) (57:43 rotamer ratio) $\delta$ $167.4\left(\mathrm{~d}, J=14.7 \mathrm{~Hz}\right.$, major $\left.\mathrm{C}_{\alpha}-\mathrm{CO}_{2}\right), 167.2(\mathrm{~d}, J=16.1 \mathrm{~Hz}$, minor $\mathrm{C}_{\alpha}-\mathrm{CO}_{2}$ ), 153.9 (minor $\left.\mathrm{CO}_{2} \mathrm{C}\left(\mathrm{CH}_{3}\right)_{3}\right), 153.5$ (major $\mathrm{CO}_{2} \mathrm{C}\left(\mathrm{CH}_{3}\right)_{3}$ ), 135.13 (minor $\underline{\mathrm{C}}_{\mathrm{q}, \mathrm{Ph}}$ ), 135.05 (major $\underline{\mathrm{C}}_{\mathrm{q}, \mathrm{Ph}}$ ), $128.61+128.58+128.52$ $+128.46+128.4+128.1$ (major and minor overlap, $\mathrm{CH}_{\mathrm{Ph}}$ ), $95.2(\mathrm{dd}$, $J=182.7,27.1 \mathrm{~Hz}$, major $\left.\mathrm{C}_{\beta}\right), 94.1(\mathrm{dd}, J=186.7,32.7 \mathrm{~Hz}$, minor $\left.\mathrm{C}_{\beta}\right), 92.1\left(\mathrm{dd}, J=179.0,32.3 \mathrm{~Hz}\right.$, minor $\left.\mathrm{C}_{\gamma}\right), 91.2(\mathrm{dd}, J=179.0,31.5$ $\mathrm{Hz}$, major $\mathrm{C}_{\gamma}$ ), 81.0 (major and minor overlap, $\left.\mathrm{CO}_{2} \underline{\mathrm{C}}\left(\mathrm{CH}_{3}\right)_{3}\right), 67.5$ (major and minor overlap, $\left.\underline{\mathrm{C}}_{2} \mathrm{Ph}\right), 64.3\left(\mathrm{~d}, J=23.5 \mathrm{~Hz}\right.$, major $\mathrm{C}_{\alpha}$ ), $63.9\left(\mathrm{~d}, J=23.5 \mathrm{~Hz}\right.$, minor $\left.\mathrm{C}_{\alpha}\right), 50.8\left(\mathrm{~d}, J=23.5 \mathrm{~Hz}\right.$, minor $\left.\mathrm{C}_{\delta}\right), 50.4$ $\left(\mathrm{d}, J=23.5 \mathrm{~Hz}\right.$, major $\left.\mathrm{C}_{\delta}\right), 28.3\left(\right.$ minor $\left.\mathrm{CO}_{2} \mathrm{C}\left(\mathrm{CH}_{3}\right)_{3}\right), 28.1$ (major $\left.\mathrm{CO}_{2} \mathrm{C}\left(\mathrm{CH}_{3}\right)_{3}\right)$ ppm; ${ }^{19} \mathrm{~F}$ NMR $\left(376 \mathrm{MHz}, \mathrm{CDCl}_{3}\right) \delta-187.5$ to 
$-188.7\left(\mathrm{~m}, 1 \mathrm{~F}\right.$ major $\mathrm{F}+1 \mathrm{~F}$ minor $\mathrm{F}+1 \mathrm{~F}$ major $\mathrm{F}^{\prime}+1 \mathrm{~F}$ minor $\left.\mathrm{F}^{\prime}\right)$ ppm; ${ }^{19} \mathrm{~F}\left\{{ }^{1} \mathrm{H}\right\}$ NMR $\left(376 \mathrm{MHz}, \mathrm{CDCl}_{3}\right)$ (57:43 rotamer ratio) $\delta$ -187.7 (d, $J=13.9 \mathrm{~Hz}, 1 \mathrm{~F}$, major $\mathrm{F}),-188.0(\mathrm{~d}, J=15.6 \mathrm{~Hz}, 1 \mathrm{~F}$, minor F), $-188.1\left(\mathrm{~d}, J=13.9 \mathrm{~Hz}, 1 \mathrm{~F}\right.$, major $\left.\mathrm{F}^{\prime}\right),-188.6(\mathrm{~d}, J=13.9$ $\mathrm{Hz}, 1 \mathrm{~F}$, minor $\mathrm{F}^{\prime}$ ) ppm; $R_{f} 0.56$ (hexane/acetone $\left.70: 30\right)$; $[\alpha]_{\mathrm{D}}^{22}-32.7$ (c 1.0, $\left.\mathrm{CHCl}_{3}, 22^{\circ} \mathrm{C}\right)$; MS (ESI) $(\mathrm{m} / z) 364.3[\mathrm{M}+\mathrm{Na}]^{+}$; HRMS (ESI) for $\mathrm{C}_{17} \mathrm{H}_{21} \mathrm{~F}_{2} \mathrm{NNaO}_{4}[\mathrm{M}+\mathrm{Na}]^{+}$calcd for 364.1331, found 364.1330; IR 1762 (m), 1705 (s), $1396(\mathrm{~s}), 1368(\mathrm{~m}), 1168(\mathrm{~s}) \mathrm{cm}^{-1}$.

$\mathrm{N}$-(tert-Butoxycarbonyl)-(2R,3S,4S)-3,4-difluoroproline ( $N$ Boc-5) (Scheme 9). To a solution of $23 \mathrm{a}(161.0 \mathrm{mg}, 0.472 \mathrm{mmol})$ in methanol $(3.0 \mathrm{~mL})$ was added $10 \% \mathrm{Pd} / \mathrm{C}(20.0 \mathrm{mg})$. The mixture was purged with one balloon volume of hydrogen gas. Subsequently, the mixture was kept under a hydrogen atmosphere and stirred at room temperature. After $18 \mathrm{~h}$, the mixture was filtered through a plug of Celite and the solvent evaporated. Carboxylic acid ( $N$-Boc)-5 (118.5 mg, quantitative) was obtained as a clear oil. The product was used as such in the next reaction.

$N$-(Acetyl)-(2R,3S,4S)-3,4-difluoroproline Methyl Ester (21) (Scheme 9). Carboxylic acid ( $N$-Boc)-5 (118.5 mg, $0.472 \mathrm{mmol}$ ) was dissolved in methanol $(2.0 \mathrm{~mL})$, and the mixture was cooled to $0{ }^{\circ} \mathrm{C}$. Acetyl chloride $(0.167 \mathrm{~mL}, 2.360 \mathrm{mmol})$ was added dropwise, and stirring at $0{ }^{\circ} \mathrm{C}$ was continued. After $40 \mathrm{~min}$, the mixture was allowed to warm to room temperature. After $22 \mathrm{~h}$, the mixture was concentrated by rotary evaporation to yield an intermediate salt. The salt was redissolved in DCM $(3.0 \mathrm{~mL})$, the mixture was cooled to $0{ }^{\circ} \mathrm{C}$, and triethylamine $(0.132 \mathrm{~mL}, 0.944 \mathrm{mmol})$ was added. Next, acetyl chloride $(0.100 \mathrm{~mL}, 1.416 \mathrm{mmol})$ was added dropwise, and the solution was allowed to warm room temperature. After stirring for 24 $\mathrm{h}$, the mixture was poured into water $(10 \mathrm{~mL})$ and the aqueous layer was extracted with DCM $(4 \times 10 \mathrm{~mL})$. The combined organic layers were dried over $\mathrm{MgSO}_{4}$ and evaporated in vacuo. The crude product was purified by flash chromatography (hexane/acetone 70:30) to yield $21(29.3 \mathrm{mg}, 30 \%)$ as a colorless solid: ${ }^{1} \mathrm{H}$ NMR $(400 \mathrm{MHz}$, $\left.\mathrm{CDCl}_{3}\right)\left(80: 20\right.$ rotamer ratio) $\delta 5.53-5.09\left(\mathrm{~m}, 1 \mathrm{H}\right.$ major $\mathrm{C}_{\beta} \mathrm{H}+1 \mathrm{H}$ minor $\mathrm{C}_{\beta} \mathrm{H}+1 \mathrm{H}$ major $\mathrm{C}_{\gamma} \mathrm{H}+1 \mathrm{H}$ minor $\left.\mathrm{C}_{\gamma} \mathrm{H}\right), 4.92-4.73(\mathrm{~m}, 1 \mathrm{H}$ major $\mathrm{C}_{\alpha} \mathrm{H}+1 \mathrm{H}$ minor $\left.\mathrm{C}_{\alpha} \mathrm{H}\right), 4.35-3.59\left(\mathrm{~m}, 2 \mathrm{H}\right.$ major $\mathrm{C}_{\delta} \mathrm{H}_{2}+2 \mathrm{H}$ minor $\left.\mathrm{C}_{\delta} \mathrm{H}_{2}\right), 3.85\left(\mathrm{~s}, 3 \mathrm{H}\right.$, minor $\left.\mathrm{CO}_{2} \mathrm{CH}_{3}\right), 3.80$ (s, $3 \mathrm{H}$, major $\mathrm{CO}_{2} \mathrm{CH}_{3}$ ), 2.14 (s, $3 \mathrm{H}$, major $\left.\mathrm{NCOCH}_{3}\right), 1.98$ (s, $3 \mathrm{H}$, minor $\left.\mathrm{NCOCH_{3 }}\right)$ ppm; ${ }^{13} \mathrm{C}\left\{{ }^{1} \mathrm{H}\right\} \mathrm{NMR}\left(100 \mathrm{MHz}, \mathrm{CDCl}_{3}\right)(80: 20$ rotamer ratio) $\delta 169.9\left(\right.$ minor $\left.\mathrm{NCOCH}_{3}\right), 169.5\left(\right.$ major $\left.\mathrm{NCOCH}_{3}\right), 166.9(\mathrm{~d}$, $J=8.8 \mathrm{~Hz}$, minor $\left.\mathrm{C}_{\alpha}-\mathrm{CO}_{2}\right), 166.2\left(\mathrm{~d}, J=8.1 \mathrm{~Hz}\right.$, major $\left.\mathrm{C}_{\alpha}-\mathrm{CO}_{2}\right)$, $93.5\left(\mathrm{dd}, J=187.8,33.0 \mathrm{~Hz}\right.$, minor $\left.\mathrm{C}_{\beta}\right), 91.9(\mathrm{dd}, J=183.4,30.8 \mathrm{~Hz}$, major $\left.\mathrm{C}_{\gamma}\right), 91.4\left(\mathrm{dd}, J=186.0,32.7 \mathrm{~Hz}\right.$, major $\left.\mathrm{C}_{\beta}\right), 90.5(\mathrm{dd}, J=$ $181.2,30.8 \mathrm{~Hz}$, minor $\left.\mathrm{C}_{\gamma}\right), 62.9\left(\mathrm{~d}, J=22.0 \mathrm{~Hz}\right.$, minor $\left.\mathrm{C}_{\alpha}\right), 61.5(\mathrm{~d}, J$ $=22.0 \mathrm{~Hz}$, major $\left.\mathrm{C}_{\alpha}\right), 53.1$ (minor $\left.\mathrm{CO}_{2} \mathrm{CH}_{3}\right), 52.7$ (major $\left.\mathrm{CO}_{2} \mathrm{CH}_{3}\right)$, $51.3\left(\mathrm{~d}, J=22.0 \mathrm{~Hz}\right.$, major $\left.\mathrm{C}_{\delta}\right), 49.8\left(\mathrm{~d}, J=22.0 \mathrm{~Hz}\right.$, minor $\left.\mathrm{C}_{\delta}\right), 22.1$ $\left(\right.$ major $\left.\mathrm{NCOCH}_{3}\right), 21.6\left(\right.$ minor $\left.\mathrm{NCOCH}_{3}\right)$ ppm; ${ }^{19} \mathrm{~F}$ NMR (376 $\left.\mathrm{MHz}, \mathrm{CDCl}_{3}\right)(80: 20$ rotamer ratio) $\delta-192.2$ to $-192.8(\mathrm{~m}, 1 \mathrm{~F}$, major F), -193.4 to $-193.9(\mathrm{~m}, 1 \mathrm{~F}$, minor $\mathrm{F}),-194.6$ to -195.0 (m, $1 \mathrm{~F}$, minor $\left.\mathrm{F}^{\prime}\right),-196.4$ to $-196.9\left(\mathrm{~m}, 1 \mathrm{~F}\right.$, major $\left.\mathrm{F}^{\prime}\right) \mathrm{ppm} ;{ }^{19} \mathrm{~F}\left\{{ }^{1} \mathrm{H}\right\}$ $\operatorname{NMR}\left(376 \mathrm{MHz}, \mathrm{CDCl}_{3}\right)(80: 20$ rotamer ratio) $\delta-192.6(\mathrm{~d}, J=13.9$ $\mathrm{Hz}, 1 \mathrm{~F}$, major F), -193.8 (d, $J=13.9 \mathrm{~Hz}, 1 \mathrm{~F}$, minor F), -194.9 (d, $J$ $=13.9 \mathrm{~Hz}, 1 \mathrm{~F}$, minor $\left.\mathrm{F}^{\prime}\right),-196.8\left(\mathrm{~d}, J=13.9 \mathrm{~Hz}, 1 \mathrm{~F}\right.$, major $\left.\mathrm{F}^{\prime}\right)$ ppm; $R_{f} 0.19$ (hexane/acetone 70:30); $[\alpha]_{\mathrm{D}}^{22}-47.8\left(c 0.9, \mathrm{CHCl}_{3}\right) ; \mathrm{MS}$ (ESI) $(\mathrm{m} / z) 208.2[\mathrm{M}+\mathrm{H}]^{+}, 230.2[\mathrm{M}+\mathrm{Na}]^{+}$; HRMS (ESI) for $\mathrm{C}_{8} \mathrm{H}_{11} \mathrm{~F}_{2} \mathrm{NNaO}_{3}[\mathrm{M}+\mathrm{Na}]^{+}$calcd for 230.0599, found 230.0604; IR 1758 (s), 1653 (s), 1202 (s), 1176 (s), 1048 (s), 1039 (s) $\mathrm{cm}^{-1}$.

$N$-(tert-Butoxycarbonyl)-(2R,3R,4R)-3,4-difluoroproline ( $N$ Boc-6) (Scheme 9). To a solution of $24 \mathrm{a}(64.0 \mathrm{mg}, 0.187 \mathrm{mmol})$ in methanol $(2.0 \mathrm{~mL})$ was added $10 \% \mathrm{Pd} / \mathrm{C}(10.0 \mathrm{mg})$. The mixture was purged with one balloon volume of hydrogen gas. Subsequently, the mixture was kept under a hydrogen atmosphere and stirred at room temperature. After $23 \mathrm{~h}$, the mixture was filtered through a plug of Celite and the solvent evaporated. Carboxylic acid (N-Boc)-6 (47.3 $\mathrm{mg}$, quantitative) was obtained as a clear oil. The product was used as such in the next reaction.

$N$-(Acetyl)-(2R,3R,4R)-3,4-difluoroproline Methyl Ester (22) (Scheme 9). Carboxylic acid ( $N$-Boc)-6 (46.4 mg, $0.185 \mathrm{mmol}$ ) was dissolved in methanol $(2.0 \mathrm{~mL})$ and cooled to $0{ }^{\circ} \mathrm{C}$. Acetyl chloride $(65.6 \mu \mathrm{L}, 0.923 \mathrm{mmol})$ was added dropwise, and the mixture was allowed to warm to room temperature. After $15 \mathrm{~h}$, the mixture was concentrated by rotary evaporation to yield an intermediate salt. The salt was redissolved in DCM $(2.0 \mathrm{~mL})$, the mixture was cooled to 0 ${ }^{\circ} \mathrm{C}$, and DIPEA $(80.6 \mu \mathrm{L}, 0.463 \mathrm{mmol})$ was added. Next, acetyl chloride $(52.6 \mu \mathrm{L}, 0.740 \mathrm{mmol})$ was added dropwise, and the solution was allowed to warm room temperature. After stirring for $22 \mathrm{~h}$, the solvent was evaporated in vacuo and the crude product was purified by flash chromatography (hexane/acetone 70:30) to yield $22(22.2$ $\mathrm{mg}, 58 \%)$ as a clear oil: ${ }^{1} \mathrm{H} \mathrm{NMR}\left(500 \mathrm{MHz}, \mathrm{CDCl}_{3}\right)(65: 35$ rotamer ratio) $\delta 5.45\left(\mathrm{dd}, J=47.5,6.3 \mathrm{~Hz}, 1 \mathrm{H}, \operatorname{minor} \mathrm{C}_{\beta} \mathrm{H}\right), 5.30(\mathrm{ddd}, J=$ $47.7,7.4,1.5 \mathrm{~Hz}, 1 \mathrm{H}$, major $\left.\mathrm{C}_{\beta} \mathrm{H}\right), 5.31-5.11\left(\mathrm{~m}, 1 \mathrm{H}\right.$ major $\mathrm{C}_{\gamma} \mathrm{H}+$ $1 \mathrm{H}$ minor $\left.\mathrm{C}_{\gamma} \mathrm{H}\right), 4.97\left(\mathrm{~d}, J=23.7 \mathrm{~Hz}, 1 \mathrm{H}\right.$, major $\left.\mathrm{C}_{\alpha} \mathrm{H}\right), 4.67(\mathrm{~d}, J=$ $21.1 \mathrm{~Hz}, 1 \mathrm{H}$, minor $\left.\mathrm{C}_{\alpha} \mathrm{H}\right), 4.05-3.88\left(\mathrm{~m}, 2 \mathrm{H}\right.$ major $\mathrm{C}_{\delta} \mathrm{H}_{2}+2 \mathrm{H}$ minor $\left.\mathrm{C}_{\delta} \mathrm{H}_{2}\right), 3.83\left(\mathrm{~s}, 3 \mathrm{H}\right.$, minor $\left.\mathrm{CO}_{2} \mathrm{CH}_{3}\right), 3.78$ (s, $3 \mathrm{H}$, major $\left.\mathrm{CO}_{2} \mathrm{CH}_{3}\right), 2.16\left(\mathrm{~s}, 3 \mathrm{H}\right.$, major $\left.\mathrm{NCOCH}_{3}\right), 2.07$ (s, 3H, minor $\left.\mathrm{NCOCH}_{3}\right)$ ppm; ${ }^{13} \mathrm{C}\left\{{ }^{1} \mathrm{H}\right\}$ NMR $\left(126 \mathrm{MHz}, \mathrm{CDCl}_{3}\right)(65: 35$ rotamer ratio) $\delta 170.1$ (minor $\left.\mathrm{NCOCH}_{3}\right), 169.7$ (major $\left.\mathrm{NCOCH}_{3}\right), 167.0$ (minor $\mathrm{C}_{\alpha}{ }^{-} \underline{\mathrm{CO}}_{2}$ ), 166.9 (major $\mathrm{C}_{\alpha}-\mathrm{CO}_{2}$ ), $95.1(\mathrm{dd}, J=187.1,32.7$ $\mathrm{Hz}$, minor $\left.\mathrm{C}_{\beta}\right), 93.5\left(\mathrm{dd}, J=180.3,31.3 \mathrm{~Hz}\right.$, major $\left.\mathrm{C}_{\beta}\right), 92.1(\mathrm{dd}, J=$ $172.5,31.9 \mathrm{~Hz}$, major $\mathrm{C}_{\gamma}$ ), $90.4\left(\mathrm{dd}, J=178.0,31.8 \mathrm{~Hz}\right.$, minor $\mathrm{C}_{\gamma}$ ), $64.8\left(\mathrm{~d}, J=22.7 \mathrm{~Hz}\right.$, minor $\left.\mathrm{C}_{\alpha}\right), 63.4\left(\mathrm{~d}, J=23.6 \mathrm{~Hz}\right.$, major $\left.\mathrm{C}_{\alpha}\right), 53.3$ (minor $\left.\mathrm{CO}_{2} \mathrm{CH}_{3}\right), 52.9\left(\right.$ major $\left.\mathrm{CO}_{2} \mathrm{CH}_{3}\right), 51.6(\mathrm{~d}, \mathrm{~J}=23.6 \mathrm{~Hz}$, major $\left.\mathrm{C}_{\delta}\right), 50.7\left(\mathrm{~d}, J=23.6 \mathrm{~Hz}\right.$, minor $\left.\mathrm{C}_{\delta}\right), 22.1\left(\right.$ major $\left.\mathrm{NCOCH}_{3}\right), 22.0$ (minor $\left.\mathrm{NCOCH}_{3}\right)$ ppm; ${ }^{19} \mathrm{~F}$ NMR (471 $\mathrm{MHz}, \mathrm{CDCl}_{3}$ ) (66:34 rotamer ratio) $\delta-187.9$ to $-187.3(\mathrm{~m}, 1 \mathrm{~F}$, minor $\mathrm{F}),-187.5$ to $-187.9(\mathrm{~m}, 1 \mathrm{~F}$, major $\mathrm{F}),-187.9$ to $-188.2\left(\mathrm{~m}, 1 \mathrm{~F}\right.$, major $\left.\mathrm{F}^{\prime}\right)$, -188.8 to $-189.1\left(\mathrm{~m}, 1 \mathrm{~F}\right.$, minor $\left.\mathrm{F}^{\prime}\right) \mathrm{ppm} ;{ }^{19} \mathrm{~F}\left\{{ }^{1} \mathrm{H}\right\}$ NMR $(471 \mathrm{MHz}$, $\left.\mathrm{CDCl}_{3}\right)(66: 34$ rotamer ratio) $\delta-187.1(\mathrm{~d}, J=14.8 \mathrm{~Hz}, 1 \mathrm{~F}$, minor $\mathrm{F})$, $-187.7(\mathrm{~d}, J=14.9 \mathrm{~Hz}, 1 \mathrm{~F}$, major F), -188.1 (d, $J=14.8 \mathrm{~Hz}, 1 \mathrm{~F}$, major $\left.\mathrm{F}^{\prime}\right),-189.0\left(\mathrm{~d}, J=14.8 \mathrm{~Hz}, 1 \mathrm{~F}\right.$, minor $\left.\mathrm{F}^{\prime}\right) \mathrm{ppm} ; R_{f} 0.24$ (hexane/acetone 70:30); $[\alpha]_{\mathrm{D}}^{22}-67.2\left(c 1.1, \mathrm{CHCl}_{3}\right) ; \mathrm{MS}$ (ESI) $(\mathrm{m} /$ z) $208.2[\mathrm{M}+\mathrm{H}]^{+}$; HRMS (ESI) for $\mathrm{C}_{8} \mathrm{H}_{11} \mathrm{~F}_{2} \mathrm{NNaO}_{3}[\mathrm{M}+\mathrm{Na}]^{+}$ calcd for 230.0599, found 230.0601; IR 1758 (m), 1658 (s), 1417 (m), $1208(\mathrm{~m}) \mathrm{cm}^{-1}$.

\section{ASSOCIATED CONTENT}

\section{Supporting Information}

The Supporting Information is available free of charge on the ACS Publications website at DOI: 10.1021/acs.joc.8b02920.

${ }^{1} \mathrm{H},{ }^{13} \mathrm{C}$, and ${ }^{19} \mathrm{~F}$ NMR spectra of all novel compounds, chiral HPLC chromatograms (for 3,4-dehydroproline synthesis), $J$ value analysis for epoxides $\mathbf{9 a , b}, \mathbf{d}$ and $10 a, b, d, X$-ray crystallographic data for $9 b, 21$, and 24a, computational data of the proline conformers of 21, 22, 52-55, and Ac-Pro-OMe in $\mathrm{CHCl}_{3}$ and water calculated by DFT including Gibbs free energies, electronic energy values, and Cartesian coordinates, and general NMR conditions for conformational and kinetic analysis (PDF)

Crystallographic data for compound 21 (CIF)

Crystallographic data for compound $\mathbf{9 b}$ (CIF)

Crystallographic data for compound 24a (CIF)

\section{AUTHOR INFORMATION}

\section{Corresponding Author}

*E-mail: bruno.linclau@soton.ac.uk. Fax: +44-23-8059-3781.

ORCID ${ }^{\circ}$

Ilya Kuprov: 0000-0003-0430-2682

Davy Sinnaeve: 0000-0003-2556-5895

Bruno Linclau: 0000-0001-8762-0170

\section{Present Address}

${ }^{\perp}$ D. Sinnaeve: Unité de Glycobiologie Structurale et Fonctionnelle, CNRS UMR 8576, Université de Lille, Villeneuve d'Ascq 59655, France 


\section{Notes}

The authors declare no competing financial interest.

\section{ACKNOWLEDGMENTS}

We thank the University of Southampton for funding. The Research Foundation Flanders (FWO) is indebted for a research project to J.C.M. and D.S. (3G011015), Ph.D. and postdoctoral fellowships to E.O. and D.S., respectively, and staff exchange funding (FWO-WOG Multimar). The $500 \mathrm{MHz}$ used for the kinetic analysis was funded in part by a Hercules grant from the Hercules foundation (AUGE09/006). The computational resources (Stevin Supercomputer Infrastructure) and services used in this work were provided by the VSC (Flemish Supercomputer Center), funded by Ghent University, FWO, and the Flemish Government, department EWI. The EPSRC is thanked for a partial Ph.D. grant to G.-J.H. (EPSRC-DTG EP/M50662X/1) and instrument funding (core capability EP/K039466/1).

\section{REFERENCES}

(1) Meanwell, N. A. Fluorine and Fluorinated Motifs in the Design and Application of Bioisosteres for Drug Design. J. Med. Chem. 2018, $61,5822-5880$.

(2) Aufiero, M.; Gilmour, R. Informing Molecular Design by Stereoelectronic Theory: The Fluorine Gauche Effect in Catalysis. Acc. Chem. Res. 2018, 51 (7), 1701-1710.

(3) Kirsch, P. Fluorine in liquid crystal design for display applications. J. Fluorine Chem. 2015, 177, 29-36.

(4) Zhou, Y.; Wang, J.; Gu, Z. N.; Wang, S. N.; Zhu, W.; Acena, J. L.; Soloshonok, V. A.; Izawa, K.; Liu, H. Next Generation of FluorineContaining Pharmaceuticals, Compounds Currently in Phase II-III Clinical Trials of Major Pharmaceutical Companies: New Structural Trends and Therapeutic Areas. Chem. Rev. 2016, 116 (2), 422-518.

(5) Gillis, E. P.; Eastman, K. J.; Hill, M. D.; Donnelly, D. J.; Meanwell, N. A. Applications of Fluorine in Medicinal Chemistry. J. Med. Chem. 2015, 58 (21), 8315-8359.

(6) Hunter, L. The C-F bond as a Conformational Tool in Organic and Biological Chemistry. Beilstein J. Org. Chem. 2010, 6, 38.

(7) O'Hagan, D. Understanding organofluorine chemistry. An introduction to the C-F bond. Chem. Soc. Rev. 2008, 37 (2), 308-319.

(8) Verhoork, S. J. M.; Killoran, P. M.; Coxon, C. R. Fluorinated Prolines as Conformational Tools and Reporters for Peptide and Protein Chemistry. Biochemistry 2018, 57 (43), 6132-6143.

(9) DeRider, M. L.; Wilkens, S. J.; Waddell, M. J.; Bretscher, L. E.; Weinhold, F.; Raines, R. T.; Markley, J. L. Collagen Stability: Insights from NMR Spectroscopic and Hybrid Density Functional Computational Investigations of the Effect of Electronegative Substituents on Prolyl Ring Conformations. J. Am. Chem. Soc. 2002, 124 (11), 24972505.

(10) Siebler, C.; Maryasin, B.; Kuemin, M.; Erdmann, R. S.; Rigling, C.; Grünenfelder, C.; Ochsenfeld, C.; Wennemers, H. Importance of Dipole Moments and Ambient Polarity for the Conformation of XaaPro Moieties - a Combined Experimental and Theoretical Study. Chem. Sci. 2015, 6 (12), 6725-6730.

(11) Eberhardt, E. S.; Panasik, N.; Raines, R. T. Inductive Effects on the Energetics of Prolyl Peptide Bond Isomerization: Implications for Collagen Folding and Stability. J. Am. Chem. Soc. 1996, 118 (49), 12261-12266.

(12) Panasik, N.; Eberhardt, E. S.; Edison, A. S.; Powell, D. R.; Raines, R. T. Inductive Effects on the Structure of Proline Residues. Int. J. Pept. Protein Res. 1994, 44 (3), 262-269.

(13) Renner, C.; Alefelder, S.; Bae, J. H.; Budisa, N.; Huber, R.; Moroder, L. Fluoroprolines as Tools for Protein Design and Engineering. Angew. Chem., Int. Ed. 2001, 40 (5), 923-925.

(14) Salwiczek, M.; Nyakatura, E. K.; Gerling, U. I. M.; Ye, S.; Koksch, B. Fluorinated Amino Acids: Compatibility with Native
Protein Structures and Effects on Protein-Protein Interactions. Chem. Soc. Rev. 2012, 41 (6), 2135-2171.

(15) Kubyshkin, V.; Durkin, P.; Budisa, N. Energetic Contribution to Both Acidity and Conformational Stability in Peptide Models. New J. Chem. 2016, 40 (6), 5209-5220.

(16) Gottlieb, A. A.; Fujita, Y.; Udenfriend, S.; Witkop, B. Incorporation of cis- and trans-4-Fluoro-L-prolines into Proteins and Hydroxylation of the trans Isomer During Collagen Biosynthesis. Biochemistry 1965, 4 (11), 2507-2513.

(17) Bretscher, L. E.; Jenkins, C. L.; Taylor, K. M.; DeRider, M. L.; Raines, R. T. Conformational Stability of Collagen Relies on a Stereoelectronic Effect. J. Am. Chem. Soc. 2001, 123 (4), 777-778.

(18) Holmgren, S. K.; Taylor, K. M.; Bretscher, L. E.; Raines, R. T. Code for Collagen's Stability Deciphered. Nature 1998, 392, 666.

(19) Newberry, R. W.; Raines, R. T. The n->pi* Interaction. Acc. Chem. Res. 2017, 50 (8), 1838-1846.

(20) Chow, W. Y.; Bihan, D.; Forman, C. J.; Slatter, D. A.; Reid, D. G.; Wales, D. J.; Farndale, R. W.; Duer, M. J. Hydroxyproline Ring Pucker Causes Frustration of Helix Parameters in the Collagen Triple Helix. Sci. Rep. 2015, 5, 12556.

(21) Hodges, J. A.; Raines, R. T. Stereoelectronic and Steric Effects in the Collagen Triple Helix: Toward a Code for Strand Association. J. Am. Chem. Soc. 2005, 127 (45), 15923-15932.

(22) Voronina, L.; Scutelnic, V.; Masellis, C.; Rizzo, T. R. Can Mutational Analysis Be Used To Assist Structure Determination of Peptides? J. Am. Chem. Soc. 2018, 140 (7), 2401-2404.

(23) Gee, C. T.; Arntson, K. E.; Urick, A. K.; Mishra, N. K.; Hawk, L. M. L.; Wisniewski, A. J.; Pomerantz, W. C. K. Protein-Observed 19F-NMR for Fragment Screening, Affinity Quantification and Druggability Sssessment. Nat. Protoc. 2016, 11, 1414.

(24) Urick, A. K.; Calle, L. P.; Espinosa, J. F.; Hu, H.; Pomerantz, W. C. Protein-Observed Fluorine NMR Is a Complementary Ligand Discovery Method to (1)H CPMG Ligand-Observed NMR. ACS Chem. Biol. 2016, 11 (11), 3154-3164.

(25) Kim, T. H.; Mehrabi, P.; Ren, Z.; Sljoka, A.; Ing, C.; Bezginov, A.; Ye, L. B.; Pomes, R.; Prosser, R. S.; Pai, E. F. The Role of Dimer Asymmetry and Protomer Dynamics in Enzyme Catalysis. Science 2017, 355 (6322), eaag2355.

(26) Ye, L.; Van Eps, N.; Zimmer, M.; Ernst, O. P.; Scott Prosser, R. Activation of the A2A Adenosine G-protein-coupled Receptor by Conformational Selection. Nature 2016, 533, 265.

(27) Manglik, A.; Kim, T. H.; Masureel, M.; Altenbach, C.; Yang, Z.; Hilger, D.; Lerch, M. T.; Kobilka, T. S.; Thian, F. S.; Hubbell, W. L.; Prosser, R. S.; Kobilka, B. K. Structural Insights into the Dynamic Process of beta2-Adrenergic Receptor Signaling. Cell 2015, 161 (5), $1101-1111$.

(28) Leung, R. L. C.; Robinson, M. D. M.; Ajabali, A. A. A.; Karunanithy, G.; Lyons, B.; Raj, R.; Raoufmoghaddam, S.; Mohammed, S.; Claridge, T. D. W.; Baldwin, A. J.; Davis, B. G. Monitoring the Disassembly of Virus-like Particles by (19)F-NMR. J. Am. Chem. Soc. 2017, 139 (15), 5277-5280.

(29) Thomas, C. A.; Talaty, E. R.; Bann, J. G. 3S-Fluoroproline as a Probe to Monitor Proline Isomerization during Protein Folding by 19F-NMR. Chem. Commun. 2009, No. 23, 3366-8.

(30) Pandey, A. K.; Naduthambi, D.; Thomas, K. M.; Zondlo, N. J. Proline Editing: A General and Practical Approach to the Synthesis of Functionally and Structurally Diverse Peptides. Analysis of Steric versus Stereoelectronic Effects of 4-Substituted Prolines on Conformation within Peptides. J. Am. Chem. Soc. 2013, 135 (11), $4333-4363$.

(31) Tressler, C. M.; Zondlo, N. J. (2S,4R)- and (2S,4S)-Perfluorotert-butyl 4-Hydroxyproline: Two Conformationally Distinct Proline Amino Acids for Sensitive Application in 19F NMR. J. Org. Chem. 2014, 79 (12), 5880-5886.

(32) Kawahara, K.; Nemoto, N.; Motooka, D.; Nishi, Y.; Doi, M.; Uchiyama, S.; Nakazawa, T.; Nishiuchi, Y.; Yoshida, T.; Ohkubo, T.; Kobayashi, Y. Polymorphism of Collagen Triple Helix Revealed by 19F NMR of Model Peptide [Pro-4(R)-hydroxyprolyl-Gly]3-[Pro- 
4(R)-fluoroprolyl-Gly]-[Pro-4(R)-hydroxyprolyl -Gly]3. J. Phys. Chem. B 2012, 116 (23), 6908-15.

(33) Dietz, D.; Kubyshkin, V.; Budisa, N. Applying $\gamma$-Substituted Prolines in the Foldon Peptide: Polarity Contradicts Preorganization. ChemBioChem 2015, 16 (3), 403-406.

(34) Hofman, G. J.; Ottoy, E.; Light, M. E.; Kieffer, B.; Kuprov, I.; Martins, J. C.; Sinnaeve, D.; Linclau, B. Minimising Conformational Bias in Fluoroprolines Through Vicinal Difluorination. Chem. Commun. 2018, 54 (40), 5118-5121.

(35) For a recent example, see: Doebelin, C.; He, Y.; Kamenecka, T. M. Multigram-scale Synthesis of Enantiopure 3,3-Difluoroproline. Tetrahedron Lett. 2016, 57 (50), 5658-5660.

(36) Hommel, U.; Lorthiois, E. L. J.; Maibaum, J. K.; Ostermann, N.; Randl, S. A.; Vulpetti, A. Pyrrolidine Derivatives and Their Use as Complement Pathway Modulators. WO2014/002057, 2014.

(37) Liu, Z.; Jenkinson, S. F.; Vermaas, T.; Adachi, I.; Wormald, M. R.; Hata, Y.; Kurashima, Y.; Kaji, A.; Yu, C.-Y.; Kato, A.; Fleet, G. W. J. 3-Fluoroazetidinecarboxylic Acids and trans,trans-3,4-Difluoroproline as Peptide Scaffolds: Inhibition of Pancreatic Cancer Cell Growth by a Fluoroazetidine Iminosugar. J. Org. Chem. 2015, 80 (9), 42444258.

(38) Marson, C. M.; Melling, R. C. The First Enantioselective Syntheses of Vicinal Difluoropyrrolidines and the First Catalytic Asymmetric Synthesis Mediated by the C-2 Symmetry of a -CHFCHF- Unit. Chem. Commun. 1998, 1223-1224.

(39) Testa, A.; Lucas, X.; Castro, G. V.; Chan, K. H.; Wright, J. E.; Runcie, A. C.; Gadd, M. S.; Harrison, W. T. A.; Ko, E. J.; Fletcher, D.; Ciulli, A. 3-Fluoro-4-hydroxyprolines: Synthesis, Conformational Analysis, and Stereoselective Recognition by the VHL E3 Ubiquitin Ligase for Targeted Protein Degradation. J. Am. Chem. Soc. 2018, 140, 9299-9213.

(40) Dormoy, J. R. Synthesis of L-3,4-Didehydroproline - Favored Orientation in the Key-Step Elimination-Reaction. Synthesis 1982, 1982, 753-756.

(41) Grieco, P. A.; Gilman, S.; Nishizawa, M. Organoselenium Chemistry. A Facile One-step Synthesis of Alkyl Aryl Selenides from Alcohols. J. Org. Chem. 1976, 41 (8), 1485-1486.

(42) Bernard, H.; Bülow, G.; Lange, U. E. W.; Mack, H.; Pfeiffer, T.; Schäfer, B.; Seitz, W.; Zierke, T. Technical Scale Synthesis of a New and Highly Potent Thrombin Inhibitor. Synthesis 2004, 2004 (14), 2367-2375.

(43) Chiba, J.; Takayama, G.; Takashi, T.; Yokoyama, M.; Nakayama, A.; Baldwin, J. J.; McDonald, E.; Moriarty, K. J.; Sarko, C. R.; Saionz, K. W.; Swanson, R.; Hussain, Z.; Wong, A.; Machinaga, N. Synthesis, Biological Evaluation, and Pharmacokinetic Study of Prolyl-1-Piperazinylacetic acid and Prolyl-4-Piperidinylacetic acid Derivatives as VLA-4 Antagonists. Bioorg. Med. Chem. 2006, 14 (8), $2725-2746$.

(44) Goli, D. M.; Cheesman, B. V.; Hassan, M. E.; Lodaya, R.; Slama, J. T. Synthesis of (2R,3R,4S)-2-Hydroxymethylpyrrolidine-3,4diol from (2S)-3,4-Dehydroproline Derivatives. Carbohydr. Res. 1994, 259 (2), 219-241.

(45) Priem, C.; Wuttke, A.; Berditsch, M.; Ulrich, A. S.; Geyer, A. Scaling the Amphiphilic Character and Antimicrobial Activity of Gramicidin S by Dihydroxylation or Ketal Formation. J. Org. Chem. 2017, 82 (23), 12366-12376.

(46) Jarvis, S. B. D.; Charette, A. B. Synthesis of Enantiopure Substituted Piperidines via an Aziridinium Ring Expansion. Org. Lett. 2011, 13 (15), 3830-3833.

(47) Banik, S. M.; Medley, J. W.; Jacobsen, E. N. Catalytic, Diastereoselective 1,2-Difluorination of Alkenes. J. Am. Chem. Soc. 2016, 138 (15), 5000-3.

(48) Molnar, I. G.; Gilmour, R. Catalytic Difluorination of Olefins. J. Am. Chem. Soc. 2016, 138 (15), 5004-5007.

(49) Robinson, J. K.; Lee, V.; Claridge, T. D. W.; Baldwin, J. E.; Schofield, C. J. Synthesis of (2S, 3R, 4S), (2S, 3S, 4R)-Epoxyprolines and Aminohydroxyprolines. Tetrahedron 1998, 54 (5), 981-996.
(50) Inagaki, T.; Fukuhara, T.; Hara, S. Effective Fluorination Reaction with Et3Nं3HF Under Microwave Irradiation. Synthesis 2003, 2003 (08), 1157-1159.

(51) Okoromoba, O. E.; Han, J.; Hammond, G. B.; Xu, B. Designer HF-Based Fluorination Reagent: Highly Regioselective Synthesis of Fluoroalkenes and gem-Difluoromethylene Compounds from Alkynes. J. Am. Chem. Soc. 2014, 136 (41), 14381-14384.

(52) Ueda, M.; Ono, A.; Nakao, D.; Miyata, O.; Naito, T. First Total Synthesis of Penmacric Acid and its Stereoisomer. Tetrahedron Lett. 2007, 48 (5), 841-844.

(53) Lee, S.; Kodera, Y.; Noda, K.; Aoyagi, H.; Kato, T.; Izumiya, N. Efficient Synthesis of a New Amino Acid, (2S,3R,4R)-4-Chloro-3hydroxyproline. Bull. Chem. Soc. Jpn. 1986, 59, 493-496.

(54) Baldwin, J. E.; Field, R. A.; Lawrence, C. C.; Lee, V.; Robinson, J. K.; Schofield, C. J. Substrate Specificity of Proline-4-hydroxylase: Chemical and Enzymatic Synthesis of 2S,3R,4S-Epoxyproline. Tetrahedron Lett. 1994, 35 (26), 4649-4652.

(55) Gao, Y.; Klunder, J. M.; Hanson, R. M.; Masamune, H.; Ko, S. Y.; Sharpless, K. B. Catalytic Asymmetric Epoxidation and Kinetic Resolution: Modified Procedures Including in Situ Derivatization. J. Am. Chem. Soc. 1987, 109 (19), 5765-5780.

(56) Marson, C. M.; Melling, R. C. Enantioselective Syntheses of trans-3,4-Difluoropyrrolidines and Investigation of their Applications in Catalytic Asymmetric Synthesis. J. Org. Chem. 2005, 70 (24), 9771-9779.

(57) Caldwell, C. G.; Chen, P.; He, J. F.; Parmee, E. R.; Leiting, B.; Marsilio, F.; Patel, R. A.; Wu, J. K.; Eiermann, G. J.; Petrov, A.; He, H. B.; Lyons, K. A.; Thornberry, N. A.; Weber, A. E. Fluoropyrrolidine Amides as Dipeptidyl Peptidase IV Inhibitors. Bioorg. Med. Chem. Lett. 2004, 14 (5), 1265-1268.

(58) Zhao, X.; Zhuang, W.; Fang, D.; Xue, X.; Zhou, J. A Highly Efficient Conversion of Primary or Secondary Alcohols into Fluorides with n-Perfluorobutanesulfonyl Fluoride-Tetrabutylammonium Triphenyldifluorosilicate. Synlett 2009, 2009 (05), 779-782.

(59) Pilcher, A. S.; Ammon, H. L.; DeShong, P. Utilization of Tetrabutylammonium Triphenylsilyldifluoride as a Fluoride Source for Nucleophilic Fluorination. J. Am. Chem. Soc. 1995, 117 (18), $5166-5167$

(60) Yin, J.; Zarkowsky, D. S.; Thomas, D. W.; Zhao, M. M.; Huffman, M. A. Direct and Convenient Conversion of Alcohols to Fluorides. Org. Lett. 2004, 6 (9), 1465-1468.

(61) Rey, Y. P.; Gilmour, R. Modulating NHC Catalysis with Fluorine. Beilstein J. Org. Chem. 2013, 9, 2812-2820.

(62) Barraclough, P.; Dieterich, P.; Spray, C. A.; Young, D. W. Two Separate and Distinct Syntheses of Stereospecifically Deuteriated Samples of (2S)-Proline. Org. Biomol. Chem. 2006, 4 (8), 1483-91.

(63) Barraclough, P.; Spray, C. A.; Young, D. W. Synthesis of $(2 \mathrm{~S}, 3 \mathrm{R})$ - and (2S,3S)-[3-2H1]-Proline via Highly Stereoselective Hydrolysis of a Silyl Enol Ether. Tetrahedron Lett. 2005, 46 (27), 4653-4655.

(64) Baldwin, J. E.; Adlington, R. M.; Godfrey, C. R. A.; Gollins, D. W.; Vaughan, J. G. A Novel Entry to Carbenoid Species via $\beta$ Ketosulfoxonium Ylides. J. Chem. Soc., Chem. Commun. 1993, 14341435.

(65) Barraclough, P.; Hudhomme, P.; Spray, C. A.; Young, D. W. Synthesis of Kainoid Analogues. Tetrahedron 1995, 51, 4195-4212.

(66) Qiu, X. L.; Qing, F. L. Practical Synthesis of Boc-Protected cis4-Trifluoromethyl and cis-4-Difluoromethyl-L-prolines. J. Org. Chem. 2002, 67 (20), 7162-7164.

(67) Newberry, R. W.; VanVeller, B.; Guzei, I. A.; Raines, R. T. n $\rightarrow$ $\pi^{*}$ Interactions of Amides and Thioamides: Implications for Protein Stability. J. Am. Chem. Soc. 2013, 135 (21), 7843-7846.

(68) Kim, W.; Hardcastle, K. I.; Conticello, V. P. Fluoroproline Flipflop: Regiochemical Reversal of a Stereoelectronic Effect on Peptide and Protein Structures. Angew. Chem., Int. Ed. 2006, 45 (48), 81418145.

(69) Kurtkaya, S.; Barone, V.; Peralta, J. E.; Contreras, R. H.; Snyder, J. P. On the Capriciousness of the FCCF Karplus Curve. J. Am. Chem. Soc. 2002, 124, 9702-9703. 
(70) Sinnaeve, D.; Foroozandeh, M.; Nilsson, M.; Morris, G. A. A General Method for Extracting Individual Coupling Constants from Crowded 1H NMR Spectra. Angew. Chem., Int. Ed. 2016, 55, 10901093.

(71) Crespo, M. D.; Rubini, M. Rational Design of Protein Stability: Effect of (2S,4R)-4-Fluoroproline on the Stability and Folding Pathway of Ubiquitin. PLoS One 2011, 6 (5), No. e19425.

(72) Naduthambi, D.; Zondlo, N. J. Stereoelectronic Tuning of the Structure and Stability of the Trp Cage Miniprotein. J. Am. Chem. Soc. 2006, 128 (38), 12430-12431.

(73) Nadarajan, S. P.; Deepankumar, K.; Seo, J.-H.; Yun, H. Evaluating the Role of Puckering and Fluorine Atom in Stability and Folding of Fluoroproline Containing Proteins. Biotechnol. Bioprocess Eng. 2017, 22 (5), 504-511.

(74) Roderer, D.; Glockshuber, R.; Rubini, M. Acceleration of the Rate-Limiting Step of Thioredoxin Folding by Replacement of its Conserved cis-Proline with (4S)-Fluoroproline. ChemBioChem 2015, 16 (15), 2162-2166.

(75) Torbeev, V. Y.; Hilvert, D. Both the cis-trans Equilibrium and Isomerization Dynamics of a Single Proline Amide Modulate Beta 2Microglobulin Amyloid Assembly. Proc. Natl. Acad. Sci. U. S. A. 2013, 110 (50), 20051-20056.

(76) Arnold, U.; Raines, R. T. Replacing a Single Atom Accelerates the Folding of a Protein and Increases its Thermostability. Org. Biomol. Chem. 2016, 14 (28), 6780-6785.

(77) Doerfel, L. K.; Wohlgemuth, I.; Kubyshkin, V.; Starosta, A. L.; Wilson, D. N.; Budisa, N.; Rodnina, M. V. Entropic Contribution of Elongation Factor P to Proline Positioning at the Catalytic Center of the Ribosome. J. Am. Chem. Soc. 2015, 137 (40), 12997-3006.

(78) Berger, A. A.; Voller, J. S.; Budisa, N.; Koksch, B. Deciphering the Fluorine Code-The Many Hats Fluorine Wears in a Protein Environment. Acc. Chem. Res. 2017, 50 (9), 2093-2103.

(79) Somovilla, V. J.; Bermejo, I. A.; Albuquerque, I. S.; MartínezSáez, N.; Castro-López, J.; García-Martín, F.; Compañón, I.; Hinou, H.; Nishimura, S.-I.; Jiménez-Barbero, J.; Asensio, J. L.; Avenoza, A.; Busto, J. H.; Hurtado-Guerrero, R.; Peregrina, J. M.; Bernardes, G. J. L.; Corzana, F. The Use of Fluoroproline in MUC1 Antigen Enables Efficient Detection of Antibodies in Patients with Prostate Cancer. J. Am. Chem. Soc. 2017, 139 (50), 18255-18261.

(80) Huang, K. Y.; Horng, J. C. Modulating the Affinities of Phosphopeptides for the Human Pin 1 WW Domain Using 4Substituted Proline Derivatives. Biochemistry 2015, 54 (40), 61866194.

(81) Jimenez-Moreno, E.; Jimenez-Oses, G.; Gomez, A. M.; Santana, A. G.; Corzana, F.; Bastida, A.; Jimenez-Barbero, J.; Asensio, J. L. A Thorough Experimental Study of $\mathrm{CH} / \mathrm{pi}$ Interactions in Water: Quantitative Structure-Stability Relationships for Carbohydrate/ Aromatic Complexes. Chem. Sci. 2015, 6, 6076-6085.

(82) Lu, K. P.; Finn, G.; Lee, T. H.; Nicholson, L. K. Prolyl cis-trans Isomerization as a Molecular Timer. Nat. Chem. Biol. 2007, 3, 619629.

(83) Wedemeyer, W. J.; Welker, E.; Scheraga, H. A. Proline cis-trans Isomerization and Protein Folding. Biochemistry 2002, 41 (50), 14637-14644.

(84) Schmuck, C.; Bickert, V.; Merschky, M.; Geiger, L.; Rupprecht, D.; Dudaczek, J.; Wich, P.; Rehm, T.; Machon, U. A Facile and Efficient Multi-Gram Synthesis of N-Protected 5-(Guanidinocarbonyl)-1H-pyrrole-2-carboxylic Acids. Eur. J. Org. Chem. 2008, 2008 (2), 324-329. 